\title{
Electron Paramagnetic Resonance and Electron-Nuclear Double Resonance Characterization of Point Defects in Titanium dioxide Crystals
}

\author{
Adam Brant \\ West Virginia University
}

Follow this and additional works at: https://researchrepository.wvu.edu/etd

\author{
Recommended Citation \\ Brant, Adam, "Electron Paramagnetic Resonance and Electron-Nuclear Double Resonance \\ Characterization of Point Defects in Titanium dioxide Crystals" (2011). Graduate Theses, Dissertations, \\ and Problem Reports. 3422. \\ https://researchrepository.wvu.edu/etd/3422
}

This Dissertation is protected by copyright and/or related rights. It has been brought to you by the The Research Repository @ WVU with permission from the rights-holder(s). You are free to use this Dissertation in any way that is permitted by the copyright and related rights legislation that applies to your use. For other uses you must obtain permission from the rights-holder(s) directly, unless additional rights are indicated by a Creative Commons license in the record and/ or on the work itself. This Dissertation has been accepted for inclusion in WVU Graduate Theses, Dissertations, and Problem Reports collection by an authorized administrator of The Research Repository @ WVU.

For more information, please contact researchrepository@mail.wvu.edu. 


\title{
Electron Paramagnetic resonance and Electron-Nuclear Double Resonance Characterization of Point Defects in Titanium dioxide Crystals
}

\author{
Adam Brant \\ A Dissertation \\ Submitted to the \\ Eberly College of Arts and Sciences \\ at West Virginia University \\ in partial fulfillment of the requirements \\ for the degree of \\ Doctor of Philosophy \\ in \\ Physics \\ Larry E. Halliburton, Ph.D., Chair \\ Mikel B. Holcomb, Ph.D. \\ Leonardo Golubovic, Ph.D. \\ James P. Lewis, Ph.D. \\ Charter D. Stinespring, Ph.D. \\ Department of Physics
}

Morgantown, West Virginia

2011 


\section{Abstract \\ Electron Paramagnetic Resonance and Electron-Nuclear Double Resonance Characterization of Point Defects in Titanium Dioxide Crystals}

\section{Adam Brant}

Electron paramagnetic resonance (EPR) and electron-nuclear double resonance (ENDOR) are used to characterize several point defects in titanium dioxide $\left(\mathrm{TiO}_{2}\right)$ single crystals in the rutile phase. A defect reported in 1961 by P. F. Chester called the "A Center" is assigned to a neutral hydrogen donor. Many researchers believe that the model for this $\mathrm{S}=1 / 2$ defect is an interstitial titanium ion $\left(\mathrm{Ti}^{3+}\right)$ and that $\mathrm{Ti}^{3+}$ interstitials are the most dominant shallow donor in $\mathrm{TiO}_{2}$. I show that the model for the A center is a neutral hydrogen donor and suggest that the $\mathrm{Ti}^{3+}$ interstitial model is not the most prevalent shallow donor defect in $\mathrm{TiO}_{2}$.

Substitutional $\mathrm{Cu}^{2+}$ defects that are unintentionally introduced to $\mathrm{TiO}_{2}$ (rutile) during growth are characterized and assigned to $\mathrm{Cu}^{2+}$ ion with an adjacent oxygen vacancy. Exact matrix diagonalization is used here to compute accurate values for the nuclear quadrupole parameter. The reduced intensity of the $\mathrm{Cu}^{2+} \mathrm{EPR}$ signal when the sample is illuminated with $442 \mathrm{~nm}$ laser light as well as the appearance of photoinduced EPR signals due to singly and doubly ionized oxygen vacancies provide evidence that the $\mathrm{Cu}^{2+}$ defect has an adjacent oxygen vacancy.

Interstitial lithium ions $\left(\mathrm{Li}^{+}\right)$adjacent to $\mathrm{Ti}^{3+}$ ions and substitutional $\mathrm{Fe}^{3+}$ defects $\left(\mathrm{Fe}^{3+}-\mathrm{Li}^{+}\right)$are also characterized. These defects were introduced to the rutile crystal by heating at $450{ }^{\circ} \mathrm{C}$ in $\mathrm{LiOH}$ powder for times on the order of several hours. Principal values and principal axis directions of the $g$ matrix are calculated for the interstitial $\mathrm{Li}^{+}$ ion adjacent to $\mathrm{Ti}^{3+}$ ion and photoinduced effects of the $\mathrm{Fe}^{3+}-\mathrm{Li}^{+}$defect are examined. 


\section{Acknowledgements}

First, I would like to thank my advisor, Dr. Larry Halliburton, for giving me the wonderful opportunity to work with him over the past few years. He is one of the most knowledgeable, patient and kind persons I have ever met and was a pleasure to work for. I would also like to thank Dr. Nancy Giles for the great opportunity that lies ahead at the Air Force Institute of Technology in Dayton, OH. I enjoyed working with AFIT students in the past and I look forward to working with them in the future.

I am also grateful to have worked with a number of talented colleagues at WVU; Brian Wilmer, Joe Rowley, Will Booth, Phil Tabor, Kineshma Munbodh, and Trent Johnson to name a few. They were extremely helpful with coursework and very supportive friends. I am thankful to have worked with Dr. Shan Yang; he showed me what it takes to be a good researcher and was a great person from which to learn. Everyone with whom I worked is destined to become a great physicist in the future.

On a personal level, I wish to thank my parents, Wayne and Cindy and my sister Emily for all their support over the years. None of this would have happened without them and I am very thankful to have them in my life. I also want to thank my wonderful girlfriend, Lauren, for her love and support over the past two years. She is the best thing that has ever happened to me and I look forward to sharing many more accomplishments with her for many decades to come.

Many thanks also to all my good friends from Berlin and SRU for all their support and all the good times we have had and will have in the future. There are too many to name here, but they know who they are, and I am grateful to call them all friends. 


\section{Table of Contents}

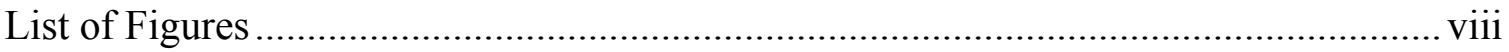

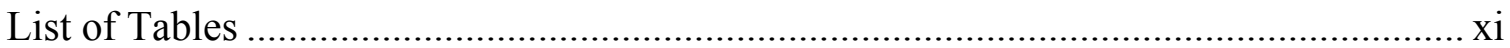

Chapter 1. Titanium Dioxide: Applications, Crystal Structure, and Growth Process ..... 1

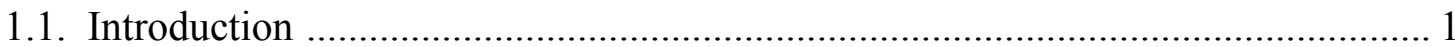

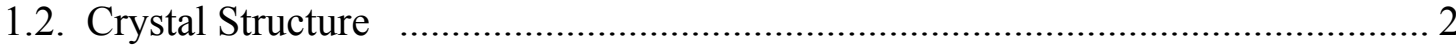

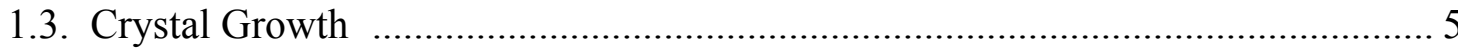

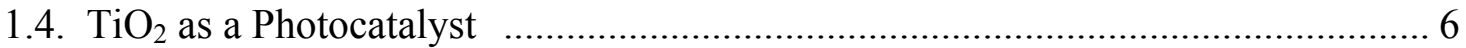

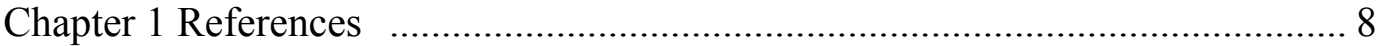

Chapter 2. Experimental and Data Analysis Techniques .............................................. 9

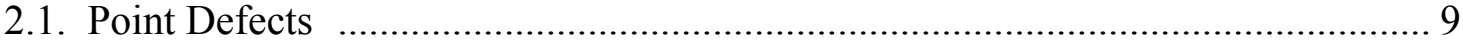

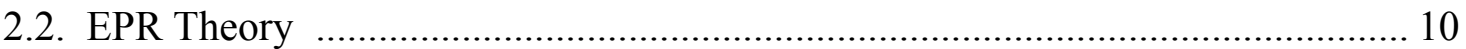

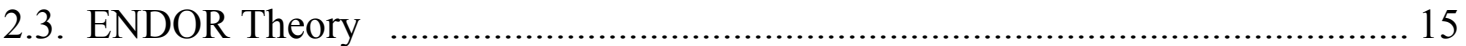

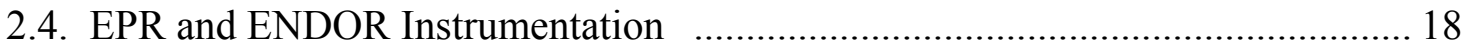

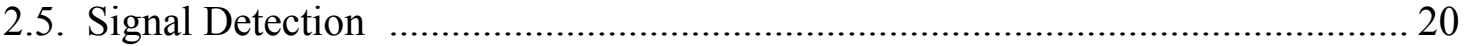

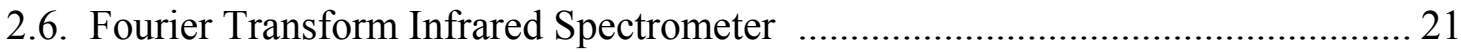

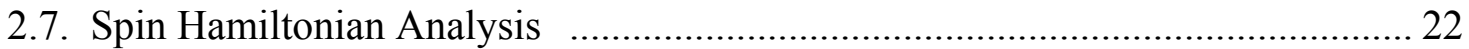

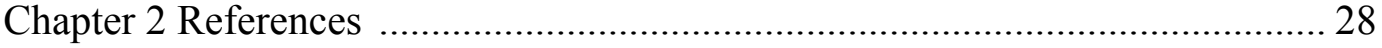

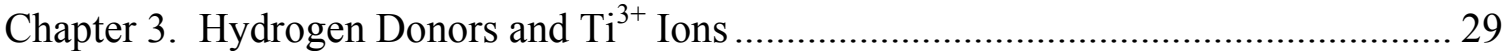

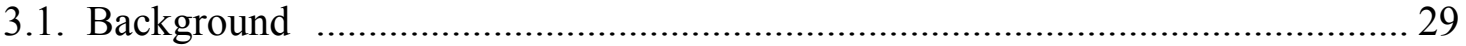

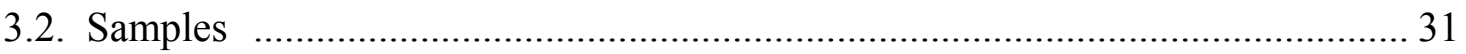

3.3. Infrared Absorption Results ...................................................................... 31

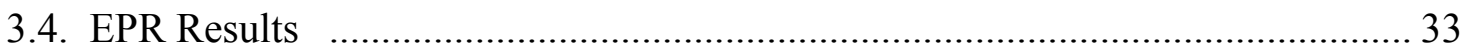

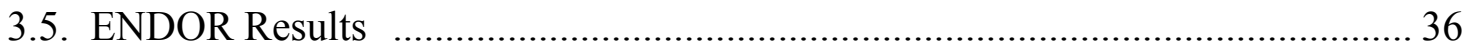

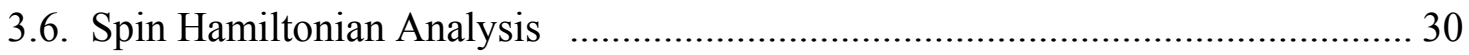

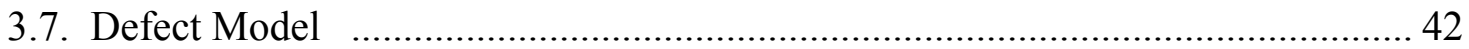

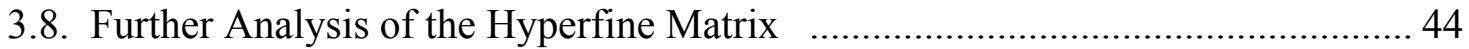

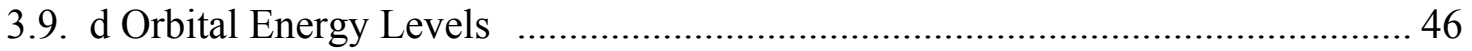

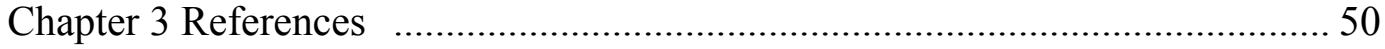

Chapter 4. Oxygen Vacancies Adjacent to Substitutional $\mathrm{Cu}^{2+}$ Ions ............................. 52

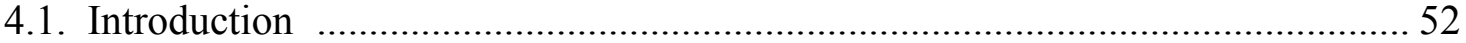

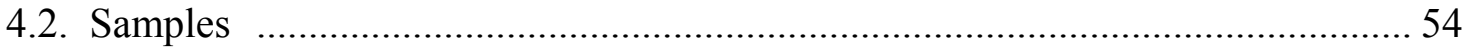

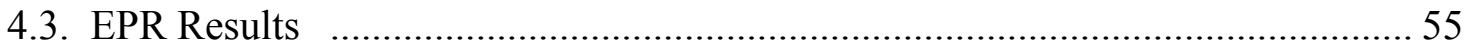

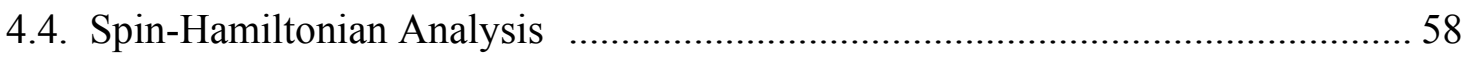

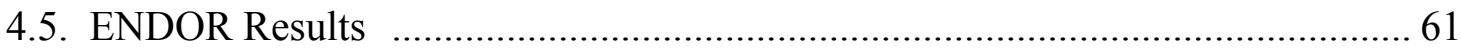

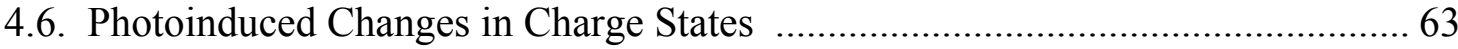

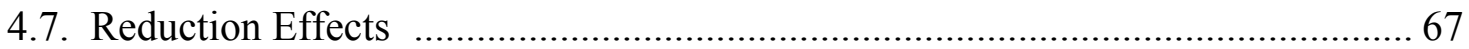




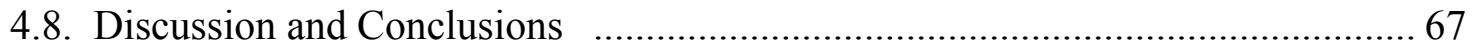

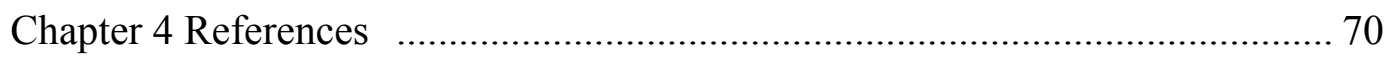

Chapter 5. Characterization of Interstitial Lithium Ions Adjacent to $\mathrm{Ti}^{3+}$ ions in $\mathrm{TiO}_{2} 72$

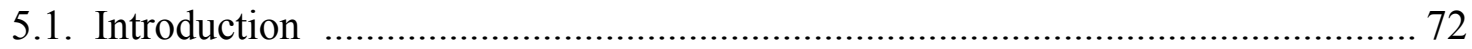

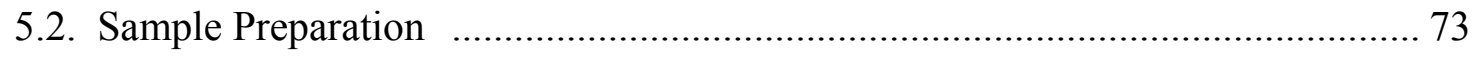

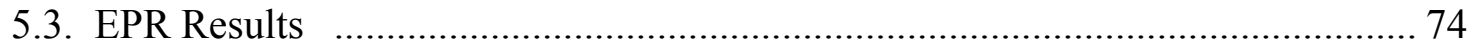

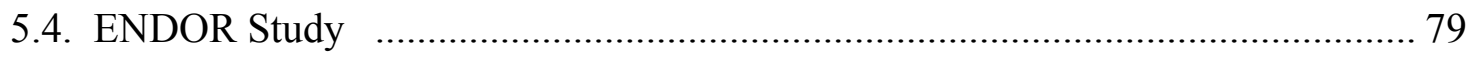

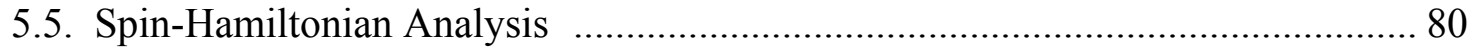

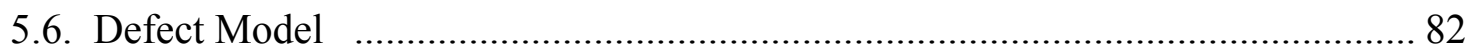

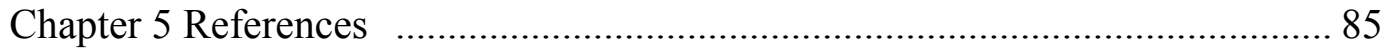

Chapter 6. Interstitial $\mathrm{Li}^{+}$Ions Adjacent to Substitutional $\mathrm{Fe}^{3+}$ Ions ……………........ 86

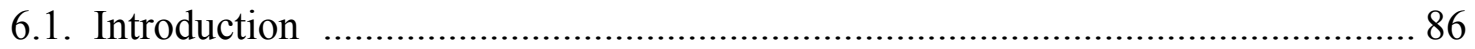

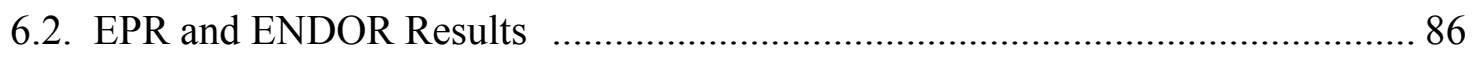

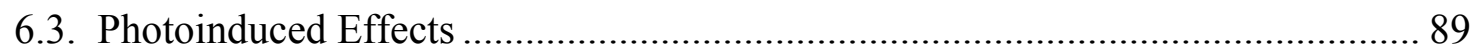

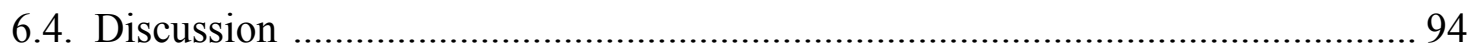

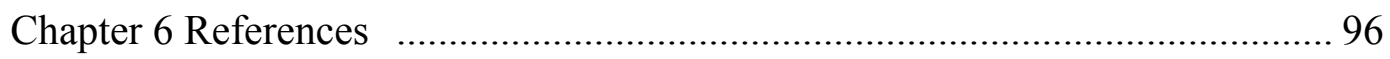

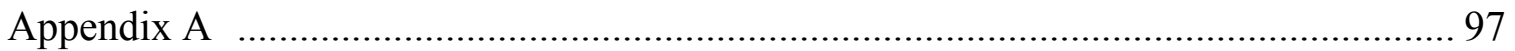

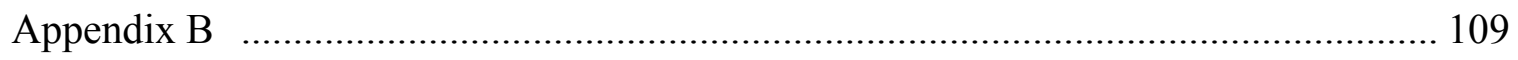

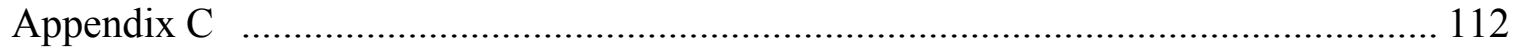




\section{Figures}

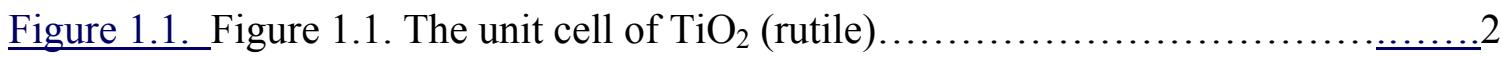

Figure 1.2. Projection on the basal plane showing the arrangement of the oxygen octahedra. .4

Figure 1.3. A schematic diagram of the apparatus used for the Verneuil growth process.5 Figure 2.1. Spin energy levels and allowed EPR transitions for an $\mathrm{S}=1 / 2, \mathrm{I}=1 / 2$ system. 15

Figure 2.2. Spin energy levels and allowed EPR and ENDOR transitions for an $\mathrm{S}=1 / 2$, I $=1 / 2$ system.

Figure 2.3. Resonant magnetic field (a) and electric field (b) components of the resonant microwave radiation in a $\mathrm{TE}_{102}$ microwave cavity . 19

Figure 2.4. Michelson interferometer used for FTIR spectroscopy.

Figure 3.1. FTIR absorption spectra of a reduced $\mathrm{TiO}_{2}$ crystal (a) at room temperature and (b) at $10 \mathrm{~K}$.

Figure 3.2. EPR spectrum of the neutral hydrogen donor taken at $5 \mathrm{~K}$ with the magnetic field along the [001] crystal axis.

Figure 3.3. EPR spectrum of the neutral hydrogen donor taken with the magnetic field aligned along (a) the [100] crystal axis, (b) $30^{\circ}$ from the [100] axis, and (c) the [110] axis.

Figure 3.4. ENDOR spectrum of the neutral hydrogen donor taken with the magnetic field along the [001] axis.

Figure 3.5. ENDOR spectrum of the neutral hydrogen donor taken with the magnetic field aligned along the [100] axis.

Figure 3.6. ENDOR spectrum of the neutral hydrogen donor taken with the magnetic field aligned along the [110] axis. 
Figure 3.7. Angular dependence of the neutral hydrogen donor EPR signal.

Figure 3.8. Angular dependence of the neutral hydrogen donor ENDOR signal.

Figure 3.9. Schematic diagram of $\mathrm{OH}^{-}$ions in rutile.

Figure 3.10. Unit cell of rutile, defining the Cartesian coordinate system that is used to determine the d-orbital energy levels.

Figure 3.11. The five d-orbital wave functions, giving the spatial distribution of valence electrons. .48

Figure 3.12. Relative energy levels of the 5 d-orbital wave functions. 49

Figure 4.1. Hysteresis curves of the as-cast and heat-treated $\mathrm{TiO}_{2}: \mathrm{Cu}$ thin films.

Figure 4.2. EPR spectrum of $\mathrm{Cu}^{2+}$ in $\mathrm{TiO}_{2}$ with the magnetic field parallel to the [001] axis.

Figure 4.3. EPR spectrum of $\mathrm{Cu}^{2+}$ in $\mathrm{TiO}_{2}$ with the magnetic field parallel to the [100] axis..

Figure 4.4. EPR spectrum of $\mathrm{Cu}^{2+}$ in $\mathrm{TiO}_{2}$ with the magnetic field parallel to the [110] axis.

Figure 4.5. EPR angular dependence of $\mathrm{Cu}^{2+}$ in $\mathrm{TiO}_{2}$.

Figure 4.6. ${ }^{63} \mathrm{Cu}$ ENDOR spectra taken with the magnetic field oriented along the [001] crystal direction.

Figure 4.7. ${ }^{63} \mathrm{Cu}$ ENDOR spectra taken with the magnetic field oriented along the [100] crystal direction.

Figure 4.8. The effect of laser illumination on $\mathrm{Cu}^{2+}$.

Figure 4.9. The effect of laser illumination on $\mathrm{Fe}^{3+}$ and $\mathrm{Cr}^{3+}$ in $\mathrm{TiO}_{2}$. 66

Figure 4.10. The $\mathrm{Cu}^{2+}-\mathrm{V}_{\mathrm{O}}$ defect model, with the pricipal axes of the $\mathrm{g}$, $\mathrm{A}$, and $\mathrm{P}$ matrices. 


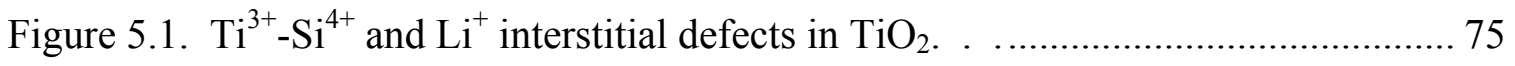

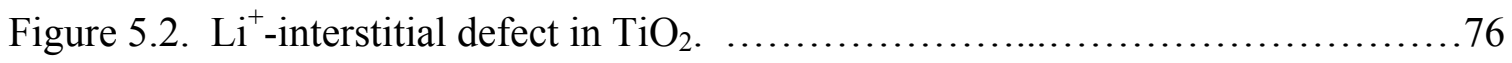

Figure 5.3. Effect of magnetic field rotation in the basal plane. . ................................. 78

Figure 5.4. ENDOR spectrum of the $\mathrm{Li}^{+}$interstitial with the field aligned along the [001]

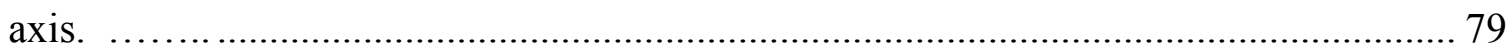

Figure 5.5. Angular dependence of the $\mathrm{Li}^{+}$interstitial in three high-symmetry planes....81

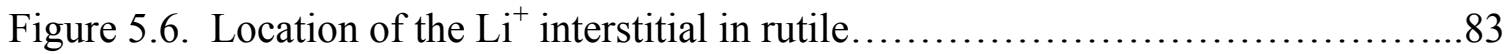

Figure 6.1. EPR spectra of a $\mathrm{Li}^{+}$ion next to an $\mathrm{Fe}^{3+}$ defect in a Japanese (a) and an as-

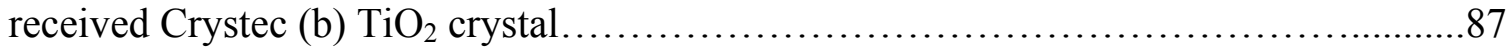

Figure 6.2. [001] axis ENDOR spectrum of $\mathrm{Li}^{+}$ions next to $\mathrm{Fe}^{3+}$ defects in a Crystec

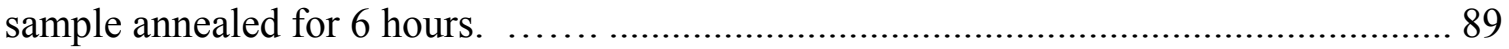

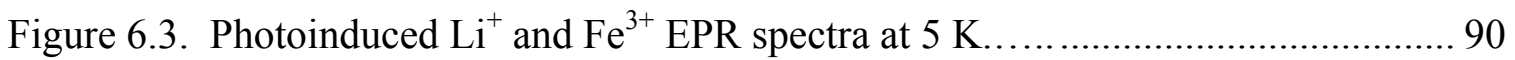

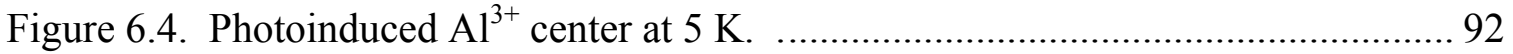

Figure B.1. Relationship between the magnetic field direction and the crystalline

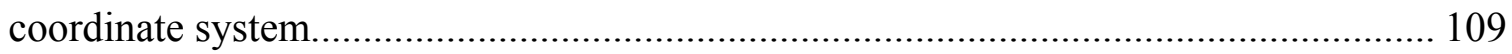

Figure B.2. Euler angles defined in the "zxz" convention............................................ 110 


\section{Tables}

Table 3.1. Principal values and principal axis directions of the $g$ and A matrices for the

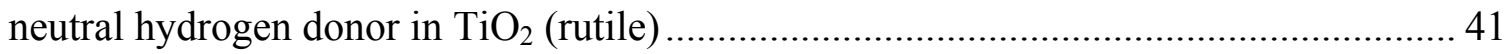

Table 4.1. Spin-Hamiltonian parameters for the two isotopes of $\mathrm{Cu}^{2+}$ in $\mathrm{TiO}_{2}$ (rutile)...60

Table 5.1. Principal values and principal axis directions for the $\mathrm{g}$ matrix of the $\mathrm{Li}^{+}$ interstitial in rutile............................................................... 81 


\section{Chapter 1}

\section{Titanium Dioxide: Applications, Crystal Structure, and Growth Process}

\subsection{Introduction}

Titanium dioxide $\left(\mathrm{TiO}_{2}\right)$ is a versatile transition-metal oxide. It is used in a wide variety of applications, including cosmetics, paints, ceramics, and as a white pigment for sunscreens. Studies of $\mathrm{TiO}_{2}$ were carried out as early as 1921 when Renz reported the reduction of the material when illuminated with sunlight in the presence of an organic compound. ${ }^{1}$ Research increased dramatically in the 1970s when Fujishima and Honda made a breakthrough discovery regarding $\mathrm{TiO}_{2}$ as a photocatalyst. ${ }^{2,3}$ The photocatalytic properties of $\mathrm{TiO}_{2}$ make it very useful for self-cleaning and deodorizing as well as gas sensing. Today, $\mathrm{TiO}_{2}$ nanotubes have emerged as an important material for energy conversion and storage, potentially to be used in solar cells and batteries. ${ }^{4}$ It has also been shown that $\mathrm{TiO}_{2}$ is a good candidate for a photoluminescent material, as it is can be a host material for rare-earth ions. ${ }^{5-9}$

In order for these applications to be useful, a full understanding of the nature of defects in $\mathrm{TiO}_{2}$ is necessary. The modification of semiconductors by adding donor and acceptor impurities is an ever-expanding field of study, and it is important that the optical and electronic properties of these defects be understood. The present dissertation is a basic study of the nature of several important point defects in bulk $\mathrm{TiO}_{2}$ (rutile) crystals using electron paramagnetic resonance (EPR) and electron nuclear double resonance (ENDOR). These experimental techniques provide a powerful means of determining the nature of point defects and their surrounding environment in the host lattice. Many studies appear each year regarding $\mathrm{TiO}_{2}$ nanotubes ${ }^{4,9-14}$ and thin films. ${ }^{5,15}$ However, 
there is still much that can be learned by doing basic defect characterization of bulk $\mathrm{TiO}_{2}$ crystals. The knowledge that is gained from these latter studies can then be applied to the nanostructured $\mathrm{TiO}_{2}$ materials and thus lead to further development of the material.

\subsection{Crystal Structure}

$\mathrm{TiO}_{2}$ occurs in three crystallographic forms: rutile, anatase, and brookite, with brookite being far less common. Rutile is the most stable form of $\mathrm{TiO}_{2}$ and is the form studied within this work. As the crystal temperature is lowered, anatase undergoes a phase change near $915^{\circ} \mathrm{C}$, irreversibly forming rutile.

Figure 1 is a diagram of the unit cell. The light grey circles represent titanium atoms while the smaller, dark grey circles represent oxygen atoms. One titanium atom lies at the center of an octahedron that is formed by the 6 surrounding oxygen atoms. Every titanium atom in $\mathrm{TiO}_{2}$ is surrounded by this oxygen octahedron. Within the unit

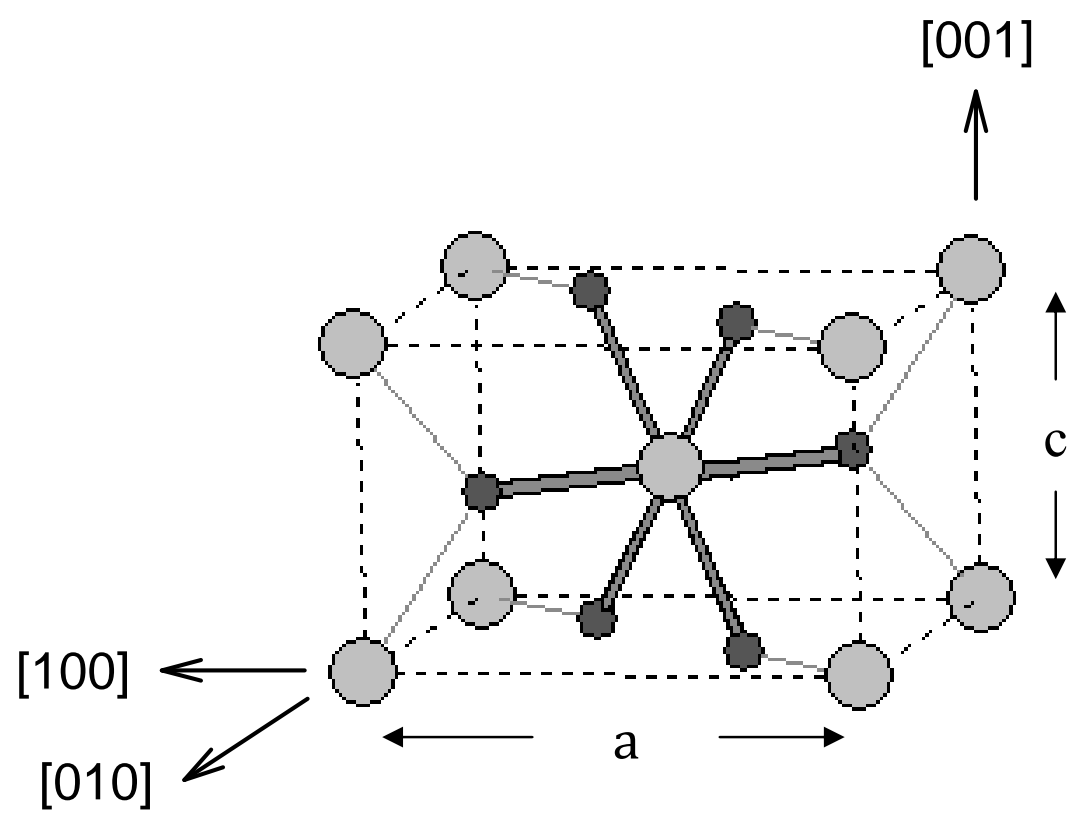

Figure 1.1. The unit cell of $\mathrm{TiO}_{2}$ (rutile). 
cell, there are four oxygen atoms and two titanium atoms; two oxygens for every titanium, hence the chemical formula $\mathrm{TiO}_{2}$. In an ionic picture, titanium exists as a $\mathrm{Ti}^{4+}$ ion, while oxygen is in its $\mathrm{O}^{2-}$ state. This figure also shows the three high-symmetry crystallographic directions in a $\mathrm{TiO}_{2}$ crystal, indicated by Miller indices. The [001] direction, or $\mathrm{c}$ axis, is the optic axis. The $\mathrm{c}$ plane (i.e., the plane perpendicular to the $\mathrm{c}$ axis) is referred to in this work as the basal plane. The base of the unit cell is square, with side lengths $\mathrm{a}=4.5937 \AA$ and the height $\mathrm{c}=2.9587 \AA$. Rutile has tetragonal symmetry and belongs to the space group $\mathrm{P} 4_{2} / \mathrm{mnm}$. This space group symbol is interpreted in the following way:

P $\quad$ - primitive cell $4_{2} / \mathrm{m}-\mathrm{a} 90^{\circ}$ rotation about the [001] direction followed by a translation of one lattice space. This is illustrated in Figure 1.2. "/m" indicates a mirror plane perpendicular to the rotation axis.

$\mathrm{nm} \quad$ - A glide along half the face diagonal with a mirror plane parallel to the glide plane.

Using the information given by the space group, the rest of the lattice can be constructed. For every lattice space along the [110] direction, the unit cell is turned $90^{\circ}$ about the [001] axis. Along the $\mathrm{c}$ axis, the unit cells are stacked repetitively on top of one another. Figure 1.2 is a projection of the lattice on the basal plane showing how the oxygen octahedra are arranged. The rotation of the unit cell along the [110] direction is clear from this diagram. The empty channels that run parallel to the [001] direction are pointed out in Fig. 1.2. These empty spaces are prime spots for the location of interstitial 


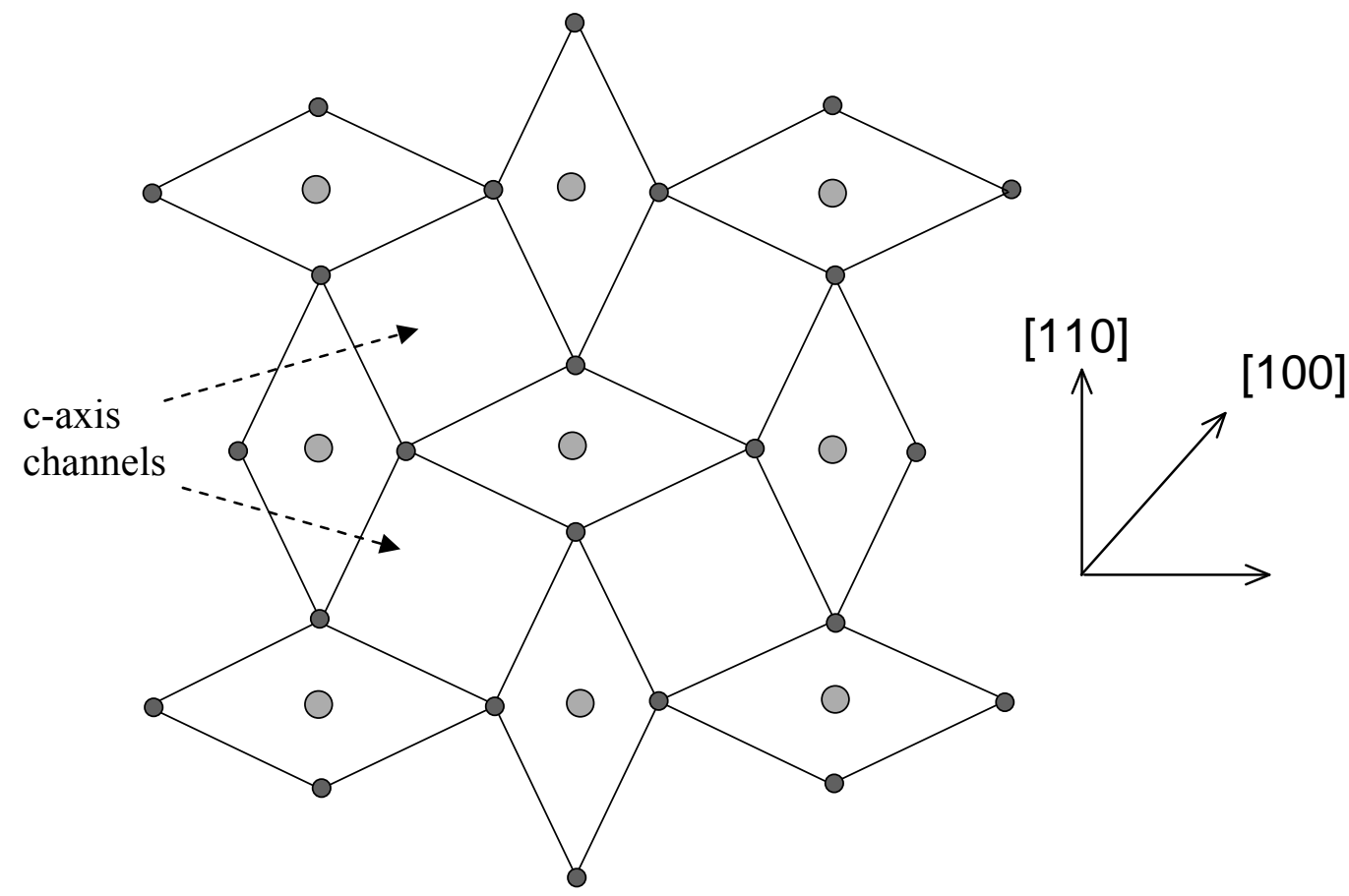

Figure 1.2. Projection on the basal plane showing the arrangement of the oxygen octahedra. The light grey circles represent titanium atoms and the smaller, dark grey circles represent oxygen atoms.

defects. Chapters 5 and 6 explore lithium interstitials that are located within these channels.

When the magnetic field is aligned along the $\mathrm{c}$ axis, the substitutional titanium sites are magnetically equivalent and the interstitial sites are magnetically equivalent. When the magnetic field is not aligned along the c axis, the two configurations of the oxygen octahedra give two magnetically inequivalent substitutional titanium sites. This means that for an arbitrary direction of the magnetic field, the field "sees" two different orientations of the oxygen octahedra at the two titanium sites. Interstitial sites have two magnetically inequivalent orientations when the field is aligned in the [110] or [100] planes and either four or eight magnetically inequivalent orientations when the magnetic field is aligned in the basal plane. 
Rutile is a direct-band-gap material, and its band gap is $3.05 \mathrm{eV}$. $\mathrm{TiO}_{2}$ (rutile) is a birefringent material, and its ordinary and extraordinary indices of refraction are given by the following dispersion relations.

$$
n_{o}^{2}=5.913+\frac{2.441 \times 10^{7}}{\lambda^{2}-0.803 \times 10^{7}} \quad n_{e}^{2}=7.197+\frac{3.322 \times 10^{7}}{\lambda^{2}-0.843 \times 10^{7}}
$$

\subsection{Crystal Growth}

There are several methods of growing $\mathrm{TiO}_{2}$ single crystals. One of these is the Verneuil process. Figure 1.3 is a schematic diagram of a Verneuil growth apparatus. This process works as follows. $\mathrm{TiO}_{2}$ powder is placed in a bin that has an opening at the bottom, out of which a tube extends. The powder can fall from the bin through this tube when the bin is vibrated. The bin has a second opening near its top so that oxygen can enter. When the bin is vibrated, the powder and oxygen simultaneously travel down through the bottom tube into an area called the Verneuil furnace. The transport tube runs within a second tube that allows hydrogen gas to enter the Verneuil furnace.

When the hydrogen and oxygen gases meet, combustion occurs, resulting in a

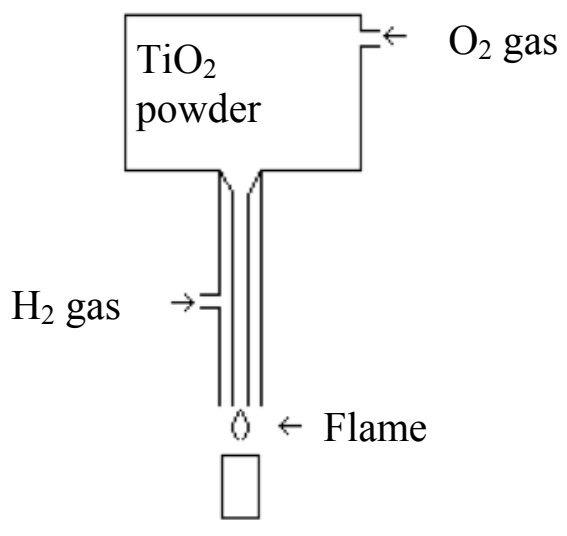

Figure 1.3. A schematic diagram of the apparatus used for the Verneuil growth process. 
flame that melts the falling $\mathrm{TiO}_{2}$ powder into droplets. This step in the process is why the Verneuil method is also known as the "flame fusion" method. The droplets fall to a support rod below, where they remain close to the flame and stay in the liquid state. This support rod is gradually pulled away from the flame, allowing the droplets to cool and crystallize. This forms a long, cylindrical crystal called a boule. Continuous pulling of the boule away from the flame allows the boule to become quite long, thus producing a substantial amount of $\mathrm{TiO}_{2}$ single crystal in one growing session. The samples in this study that are from Crystec were grown using the Verneuil method. Other processes, such as the floating zone method, are often used to grow $\mathrm{TiO}_{2}$ single crystals as well.

\section{4 $\mathrm{TiO}_{2}$ as a Photocatalyst}

The potential for this material as a photocatalyst was first discovered by Fujishima and Honda in $1972 .^{3}$ These investigators found that when $\mathrm{TiO}_{2}$ is exposed to ultraviolet light, conduction-band electron and valence-band hole pairs are formed. The reaction is as follows:

$$
h v \rightarrow e_{c b}{ }^{-}+h_{v b}^{+}
$$

In the presence of water, the valence band holes oxidize the water molecules to form $\mathrm{OH}$ radicals as follows:

$$
\mathrm{H}_{2} \mathrm{O}+h_{v b}{ }^{+} \rightarrow \bullet \mathrm{OH}+\mathrm{H}^{+}
$$

The hydroxyl radical ${ }^{\bullet} \mathrm{OH}$ is the neutral form of an $\mathrm{OH}^{-}$ion, and it has the ability to break the bonds in organic compounds, resulting in simple compounds that are easily washed away. As a result, $\mathrm{TiO}_{2}$ is added to windows, tiles, and paints to prevent the build-up of organic matter. As a photocatalyst, $\mathrm{TiO}_{2}$ also has potential as a fuel source. 
When the valence-band holes interact with water, hydrogen is separated from oxygen; a process known as hydrolysis. If the hydrogen were to be collected, it could be used as a fuel source. 


\section{Chapter 1 References}

1. C. Renz, Helvet. Chim. Acta 4, 961 (1921).

2. A. Fujishima, X. Zhang, and D. A. Tryk, Surface Science Reports, 63, 515 (2008).

3. A Fujishima, and K. Honda, Nature 238, 37 (1972).

4. B. H. Meekins and P. V. Kamat ACS Nano, 11, 3437 (2009).

5. A. Conde-Gallardo, M. Garcia-Rocha, I. Hernandez-Calderon, and R. PalominoMerino, Appl. Phys. Lett. 78, 3436 (2001).

6. S. Jeon, and P. V. Braun, Chem. Mater. 15, 1256 (2003).

7. R. Asahi, T. Morikawa, T. Ohwaki, A. Aoki, and Y. Taga, Science 293, 269 (2001).

8. B. Chi, E. S. Victorio, and T. Jin, Nanotechnology 17, 2234 (2006).

9. P. Haro-Gonzalez, M. Pedroni, F. Piccinelli, L. L. Martin, S. Polizzi, M. Giarola, G. Mariotto, A. Speghini, M. Bettinelli, and I. R. Martin, J. Lum. 131, 2473 (2011).

10. M. Adachi, Y. Murata, M. Harada, and S. Yoshikawa, Chem. Lett. 29, 942 (2000).

11. T. Kubo, M. Takeuchi, M. Matsuoka, M. Anpo, and A. Nakahira, Catal. Lett. 130, 28 (2009).

12. S. Liu, and A. Chen, Langmuir 21, 8409 (2005).

13. Y. G. Wang, and X. G. Zhang, J. Electrochem. Soc. 152, A671 (2005).

14. A. Thorne, A. Kruth, D. Tunstall, J. T. S. Irvine, and W. Zhou, J. Phys. Chem. B 109, $5439(2005)$.

15. J. Jensen, M. Mikkelsen, and F. C. Krebs, Solar Energy Materials and Solar Cells 95, 2949 (2011). 


\section{Chapter 2}

\section{Experimental and Data Analysis Techniques}

\subsection{Point Defects}

Point defects are localized imperfections within the lattice of a host material. The term "point" means that they are not extended defects such as cracks or dislocations. All crystals contain point defects. Therefore, it is important to understand how they affect the material's optical and electronic properties so that devices can operate as planned. Impurity ions can arise unintentionally as a result of impure starting materials or contamination during growth, or they can be inserted intentionally by doping. Missing atoms in the host material (vacancies), host atoms that are not on a lattice site (interstitials), and host atoms that occupy lattice sites that normally belong to a different host atom (antisites) are classified as intrinsic point defects. Any randomly distributed atoms or ions that are not part of the host material's chemical formula are called extrinsic point defects. These defects can substitute for host ions or occupy interstitial positions.

All crystals must remain electrically neutral; hence, all vacancies, interstitials, and impurities must be charge compensated. This generally occurs when electrons or holes become stabilized, or "trapped", at a defect site. For example, when the charge of a substitutional impurity ion differs from that of the host ion being replaced, it is possible for the impurity to trap an electron or hole near the impurity site in order to preserve charge neutrality. Charge trapping at defect sites leads to a spectrum of energy levels within the band gap of the material. ${ }^{1}$ Excitations between these energy levels give an array of optical absorption bands, which cause the color of the crystal to change. Thus, such defects are called "color centers." Some defects cause a crystal to emit light when 
heated or illuminated with a laser. In general, point defects are often responsible for the interesting optical and electronic properties of a material.

Point defects often have one or more unpaired spins that can be studied using EPR and ENDOR. These unpaired spins arise when electrons or holes are trapped at or near defect sites to provide charge neutrality or when the defect exists in a charge state that gives it unpaired spins in its valence shell. Many examples of these two cases exist in the literature, and each is observed in this work. Aluminum in its $3+$ charge state can substitute for a $\mathrm{Ti}^{4+}$ cation in $\mathrm{TiO}_{2}$ and trap a hole on an adjacent oxygen ion for charge compensation. A substitutional $\mathrm{Cu}^{2+}$ ion, on the other hand, has the $3 \mathrm{~d}^{9}$ electron configuration, giving it one unpaired electron in its valence shell. EPR and ENDOR are extremely useful for identifying and characterizing the paramagnetic charge state of defects.

\subsection{EPR Theory}

The two experimental techniques used in this study are electron paramagnetic resonance (EPR) and electron-nuclear double resonance (ENDOR). EPR was first demonstrated in 1944 and has since developed into a powerful tool for studying point defects in solids and for studying organic and inorganic free radicals. The EPR technique also has numerous applications in biology and medicine. These magnetic resonance techniques are used to study systems that have one or more unpaired electrons, i.e., paramagnetic systems $(S \geq 1 / 2)$. EPR and ENDOR are, therefore, very specialized techniques that are only suitable for chemical species that contained unpaired electrons. EPR is much more sensitive than similar magnetic resonance techniques such as NMR. 
With EPR, one can often detect spin concentrations of $10^{12} \mathrm{~cm}^{-3}$ or higher. The minimum number of detectable spins in an EPR cavity is $5 \times 10^{10}$ spins at $10 \mathrm{~K}$ for a one Gauss linewidth. The following empirical formula provides an approximate method of calculating the concentration of a paramagnetic defect from its EPR signal.

\section{$\mathrm{N}=5 \times 10^{10}(\mathrm{l} . \mathrm{w} .)^{2}($ Signal/Noise $)(\#$ of lines)(T/10)(1/V)}

Here, l.w. is the linewidth of the EPR signal, $\mathrm{T}$ is the sample temperature in Kelvin, and $\mathrm{V}$ is the crystal volume in cubic centimeters.

EPR can be used to study any system that is paramagnetic. As opposed to NMR, EPR detects transitions between electron spin energy levels, rather than nuclear spin energy levels. Each electron possesses a magnetic dipole moment, which can have two distinct energy levels depending on its orientation relative to an external magnetic field. The Zeeman effect describes the interaction between the spin and an external magnetic field. The energy levels of an electron spin are degenerate when there is no magnetic field present. The presence of a magnetic field lifts this degeneracy according to the orientation of the spin relative to the field, parallel orientation being lower in energy than anti-parallel. The following sketch shows the energy level splitting for a single electron $(\mathrm{S}=1 / 2)$. In general, the number of energy levels is given by $(2 \mathrm{~S}+1)$.

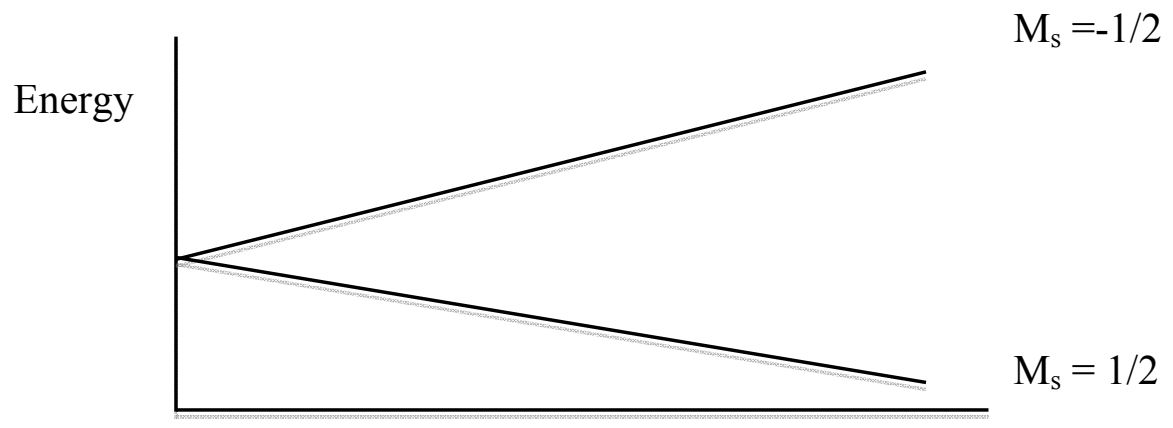

Magnetic field

Sketch of Zeeman energy levels. A spin in an external magnetic field has two distinct energy levels 
The Zeeman effect states that a magnetic dipole moment in an external magnetic field has energy $(\mathrm{U})$ given by the scalar product

$$
U=-\vec{\mu} \cdot \vec{B}
$$

One can see from the definition of the dot product that $U$ is a minimum when the dipole and magnetic field vectors are parallel and is a maximum when the two vectors are antiparallel. We will assume that the magnetic field and magnetic dipole vectors are along the $\mathrm{z}$ axis, and so $\mathrm{U}=\mu \mathrm{B}$. The dipole moment is

$$
\mu=-g \mu_{\mathrm{B}} \frac{\vec{S}}{h}=-g \mu_{\mathrm{B}} m_{s} \text {. }
$$

The quantity $\mathrm{m}_{\mathrm{s}}$ is the spin quantum number and $\mu_{\mathrm{B}}$ is the Bohr magneton. For a single electron spin, $\mathrm{m}_{\mathrm{s}}$ can have the values $1 / 2$ (parallel spin, or "spin up") or $-1 / 2$ (antiparallel, or "spin down"). This gives two distinct energy levels

$$
U= \pm \frac{1}{2} g \mu_{\mathrm{B}} B
$$

distinguished by the orientation of the moment relative to the magnetic field. The separation of the energy levels is $g \mu_{\mathrm{B}} \mathrm{B}$.

In an EPR experiment, the sample is subjected to electromagnetic radiation in the form of microwaves. The frequency of the microwaves $(v)$ is close to $9.47 \mathrm{GHz}(\mathrm{X}-$ band) and the energy of the microwave photons is hv. This frequency is held constant while the external magnetic field is swept over a specified range. At a particular field, the energy of the microwave photons will equal the separation between the Zeeman energy levels. This is referred to as the EPR resonance condition:

$$
h v=g \mu_{\beta} B
$$


It is at this resonance field where transitions can occur between the two energy levels: i.e., an electron in the spin up state can transition to the higher-energy spin down state, and then relax back down. An EPR signal appears at the resonance field. The number of EPR lines is equal to twice the value of $S$ (i.e., one line for $S=1 / 2$ ).

The preceding explanation refers to the simplest possible spin system; an isolated, single electron in an external magnetic field. Unpaired spins in crystalline solids can quite easily interact with surrounding nuclei that have a magnetic moment. The interaction of an unpaired spin with a neighboring nucleus is known as the hyperfine interaction. The hyperfine interaction splits the Zeeman energy levels into additional levels according to the nuclear spin value (I) of the neighboring nucleus. Each of the Zeeman levels is further split into $(2 \mathrm{I}+1)$ sublevels.

At particular field values that satisfy the resonance condition, more transitions can occur between sublevels, but not all transitions can occur. There are selection rules that govern which transitions take place. ${ }^{2}$ When matter interacts with electromagnetic radiation, the transition probability between initial state $\psi_{1}$ and final state $\psi_{2}$ is given by

$$
\left|\left\langle\psi_{1}\left|\hat{H}_{1}\right| \psi_{2}\right\rangle\right|^{2}
$$

$\hat{H}_{1}$ is a perturbation term representing the energy of the interaction. Consider a spin system $\mathrm{S}$ where the energy of the spin in a magnetic field is $-g \mu_{\mathrm{B}} \vec{B} \cdot \vec{S}$. In spin resonance spectroscopy, the sample is exposed to two magnetic fields: one is a timeindependent field that is taken to be along the $\mathrm{z}$ axis, and the other is the magnetic field component of electromagnetic radiation (say, $\mathrm{B}^{\prime}$ ). In an EPR experiment, this is the aforementioned X-band microwave radiation. 
Suppose the EM radiation oscillates with frequency $v$, and $\mathrm{B}^{\prime}$ is along the $\mathrm{x}$ axis, the perturbation Hamiltonian is

$$
\hat{H}_{1}=-g \mu_{\mathrm{B}} S_{\mathrm{x}} \cos [2 \pi v t]
$$

$\psi_{1}$ and $\psi_{2}$ are defined by the time-independent spin states $\left|S, m_{S 1}\right\rangle$ and $\left|S, m_{S 2}\right\rangle$, so that the transition probability between states is

$$
\left[g \mu_{\mathrm{B}} B^{\prime} \cos [2 \pi v t]\right]^{2}\left|\left\langle S, m_{s 1}\left|S_{x}\right| S, m_{s 2}\right\rangle\right|^{2}
$$

Raising and lowering spin operators are defined as

$$
S_{ \pm}=S_{\mathrm{x}} \pm i S_{\mathrm{y}}
$$

Algebraic manipulation gives

$$
S_{x}=\frac{S_{+}+S_{-}}{2}
$$

Application of $\mathrm{S}_{ \pm}$on a spin state raises/lowers the value of $\mathrm{m}_{\mathrm{s}}$ by one, so upon substitution, one gets

$$
\left\langle\psi_{1}\left|\hat{H}_{1}\right| \psi_{2}\right\rangle=\frac{N_{+}}{2}\left\langle S, m_{s 1} \mid S, m_{s 2}+1\right\rangle+\frac{N_{-}}{2}\left\langle S, m_{s 1} \mid S, m_{s 2}-1\right\rangle
$$

where $\mathrm{N}_{+}$and $\mathrm{N}_{\text {- }}$ are constants. The spin states are orthogonal, so the above equation is non-zero only if $\mathrm{m}_{\mathrm{s} 1}=\mathrm{m}_{\mathrm{s} 2} \pm 1$, giving the EPR selection rule $\Delta \mathrm{m}_{\mathrm{s}}= \pm 1$. A similar argument shows that EPR selection rule for nuclear transitions is $\Delta \mathrm{m}_{\mathrm{I}}=0$.

With selection rules in place, one can predict how many lines will appear when a specific spin S interacts with a specific nucleus I. With hyperfine interaction included, the number of line that appear in a spectrum is given by $(2 S)(2 I+1)$. For example, one electron interacting with an $I=1 / 2$ nucleus results in a two-line spectrum. Solid lines on 


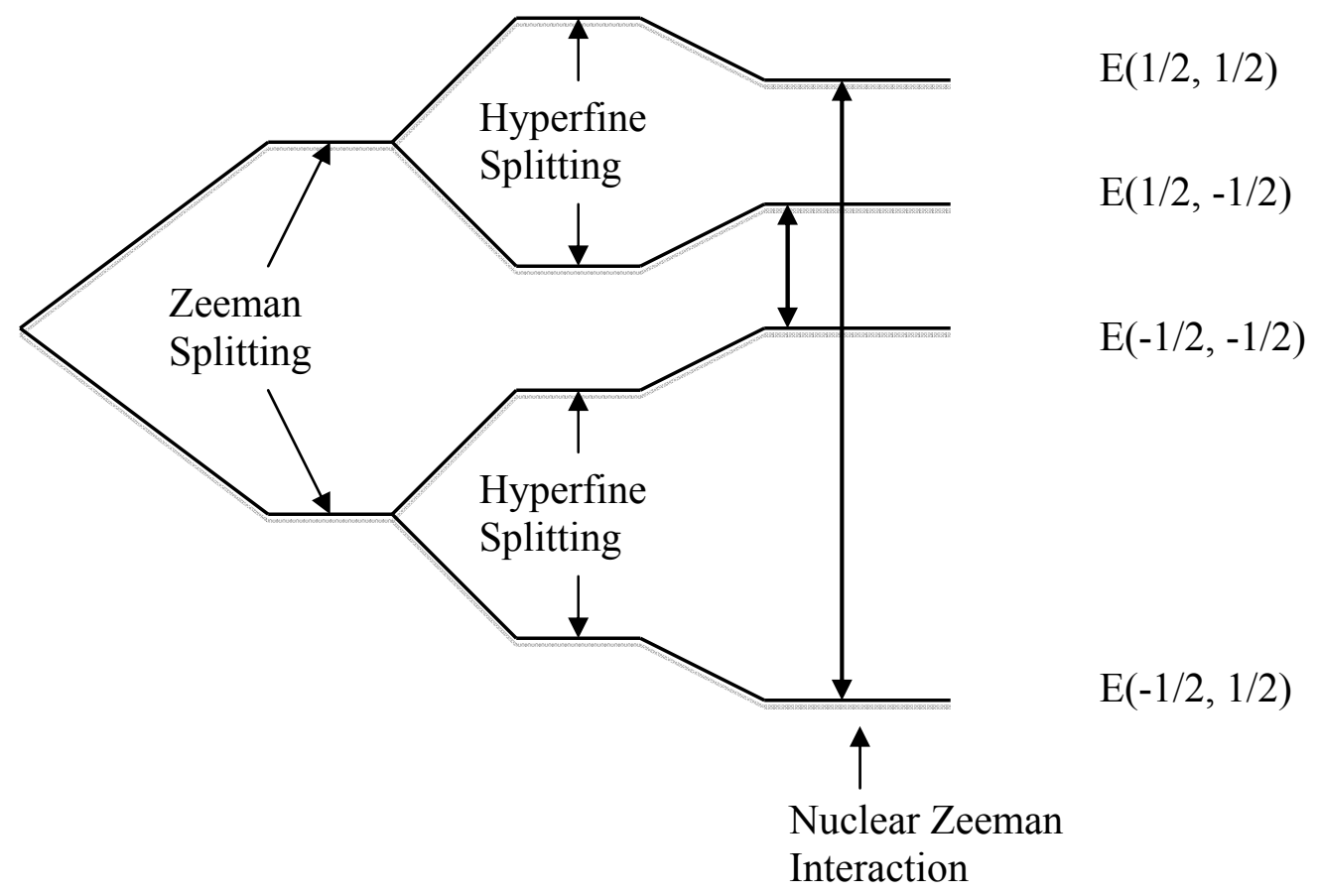

Figure 2.1. Spin energy levels and allowed EPR transitions for an $S=1 / 2, I=1 / 2$ system. Selection rules determine which EPR transitions are allowed. In this sketch, solid lines are allowed EPR transitions.

the right side of Fig. 2.1 indicate allowed EPR transitions for an $S=1 / 2, I=1 / 2$ system. This is the simplest case, and more complex cases often arise as a result of other interactions, such as nuclear quadrupole and electron-electron interactions.

\subsection{ENDOR Theory}

Electron-nuclear double resonance is a technique that is used in conjunction with an EPR experiment. Hyperfine patterns are often unresolved, or overlap with EPR signals from other defects. Quite frequently, the nucleus responsible for the observed defect cannot be definitely assigned, due to the fact that different elements can have the same nuclear spin value. These problems can be overcome using the ENDOR technique. In an ENDOR experiment, the magnetic field is held at a fixed value that corresponds to 
an EPR resonance field value. Then an $\mathrm{rf}$ frequency is applied and swept through a range of interest to drive nuclear transitions. Consider the case of one unpaired spin interacting with a nucleus with spin $I=1 / 2$. Including only the electron Zeeman, hyperfine, and nuclear Zeeman interactions, the spin Hamiltonian is, for a fixed crystal and magnetic field orientation

$$
\hat{H}=g \mu_{\mathrm{B}} \vec{B} \cdot \vec{S}+A \vec{S} \cdot \vec{I}-g_{n} \mu_{n} \vec{B} \cdot \vec{I} .
$$

Here, the values of $g$ and $\mathrm{A}$ are taken as scalars because the orientation of the crystal and field are fixed. The energy eigenvalues of this Hamiltonian are then given to first order by

$$
E\left(m_{s}, m_{I}\right)=g \mu_{\mathrm{B}} B m_{s}+A m_{s} m_{I}-g_{n} \mu_{n} B m_{I} .
$$

The two possible values of $\mathrm{m}_{\mathrm{s}}$ and $\mathrm{m}_{\mathrm{I}}$ give four different energy levels.

$$
\begin{gathered}
E\left(\frac{1}{2}, \frac{1}{2}\right)=\frac{g \mu_{\mathrm{B}} B}{2}+\frac{A}{4}-\frac{g_{n} \mu_{n} B}{2} \\
E\left(\frac{1}{2},-\frac{1}{2}\right)=\frac{g \mu_{\mathrm{B}} B}{2}-\frac{A}{4}+\frac{g_{n} \mu_{n} B}{2} \\
E\left(-\frac{1}{2}, \frac{1}{2}\right)=-\frac{g \mu_{\mathrm{B}} B}{2}-\frac{A}{4}+\frac{g_{n} \mu_{n} B}{2} \\
E\left(-\frac{1}{2},-\frac{1}{2}\right)=-\frac{g \mu_{\mathrm{B}} B}{2}+\frac{A}{4}+\frac{g_{n} \mu_{n} B}{2}
\end{gathered}
$$

These energy levels are sketched in Fig. 2.2 and show the allowed EPR transitions as dashed lines and ENDOR transitions as solid lines. The selection rules for ENDOR transitions are $\Delta \mathrm{m}_{\mathrm{S}}=0, \Delta \mathrm{m}_{\mathrm{I}}= \pm 1$. Hence, there are two allowed ENDOR transitions for an $S=1 / 2, I=1 / 2$ system, shown as solid lines in Fig. 2.2. 


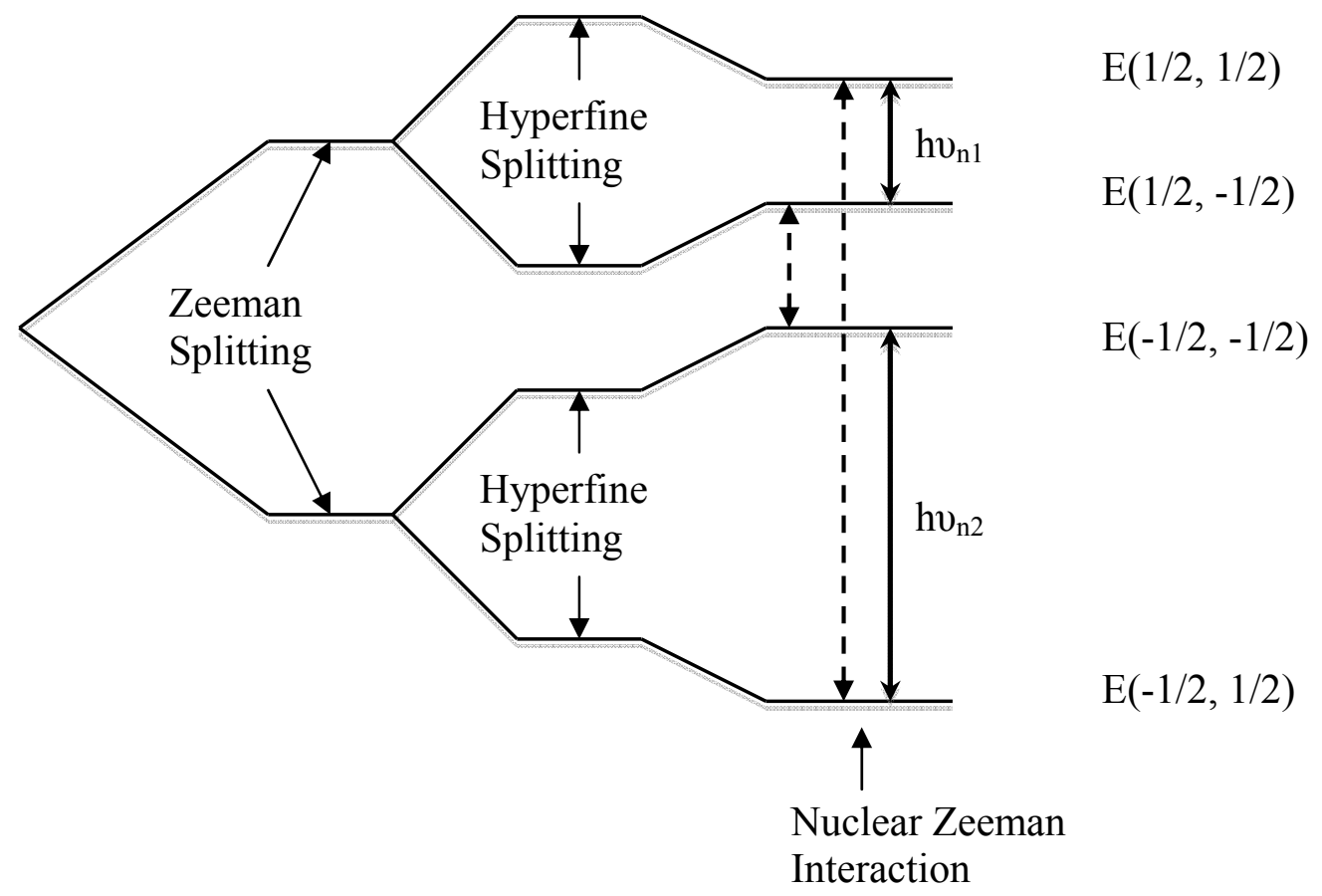

Figure 2.2. Spin energy levels and allowed EPR and ENDOR transitions for an $\mathrm{S}=$ $1 / 2, I=1 / 2$ system. Selection rules determine which EPR and ENDOR transitions are allowed. In this sketch, solid lines are allowed ENDOR transitions and dashed lines are allowed EPR transitions.

The difference between levels that give allowed ENDOR transitions are

$$
\begin{gathered}
E\left(\frac{1}{2}, \frac{1}{2}\right)-E\left(\frac{1}{2},-\frac{1}{2}\right)=\left(\left|\frac{1}{2} A-g_{n} \mu_{n} B\right|\right) \\
E\left(-\frac{1}{2},-\frac{1}{2}\right)-E\left(-\frac{1}{2}, \frac{1}{2}\right)=\left(\left|\frac{1}{2} A+g_{n} \mu_{n} B\right|\right) .
\end{gathered}
$$

The energy differences of the two allowed transitions are $h v_{\mathrm{n} 1}$ and $h v_{\mathrm{n} 2}$, hence $v_{\mathrm{ni}}$ represent the rf frequency values at which the ENDOR lines will appear. From this, it is easy to show that, when $\mathrm{A} / 2>v_{\mathrm{n}}$, the two ENDOR lines will be centered on $\mathrm{A} / 2$, enabling precise measurement of the hyperfine parameter via

$$
\frac{v_{n 2}+v_{n 1}}{2}=\frac{E\left(\frac{1}{2}, \frac{1}{2}\right)-\left(\frac{1}{2},-\frac{1}{2}\right)+E\left(-\frac{1}{2},-\frac{1}{2}\right)-E\left(-\frac{1}{2}, \frac{1}{2}\right)}{2}=\frac{A}{2} .
$$


This is particularly useful when the hyperfine interaction is too weak to be resolved with EPR.

This energy level diagram also shows how to determine the identity of the nucleus that is interacting with the unpaired electron. From the resonance condition, one can derive

$$
v_{n}=\frac{g_{n} \mu_{n} B}{\hbar}
$$

This is the ENDOR frequency of an isolated (i.e., "free") nucleus in a magnetic field. This value is known for every nucleus, as every nucleus has its own unique value of $g_{n}$. Then, from the energy level equations

$$
v_{n 2}-v_{n 1}=\frac{2 g_{n} \mu_{n} B}{\hbar}=2 v_{n}
$$

This formula shows that when $\mathrm{A} / 2>v_{\mathrm{n}}$ the two observed ENDOR lines are separated by $2 v_{N}$, making it possible to unambiguously identify the nucleus responsible for hyperfine interaction. It can be shown through a similar calculation that when $v_{\mathrm{n}}>\mathrm{A} / 2$, the two ENDOR lines are separated by A and centered on $v_{n}$.

\subsection{EPR and ENDOR Instrumentation}

The EPR apparatus used in this study consists of an electromagnet with a field range of 0 to $\sim 13,000 \mathrm{G}$. In the gap between the pole caps, there is a rectangular, metal resonance cavity that operates in the $\mathrm{TE}_{102}$ mode. The crystals are suspended inside the metal cavity with a thin brass rod. At the end of the rod, where the sample is mounted within the cavity, there is a Teflon holder, which does not absorb microwaves. The microwave cavity is connected via waveguide to the microwave bridge, which houses the 


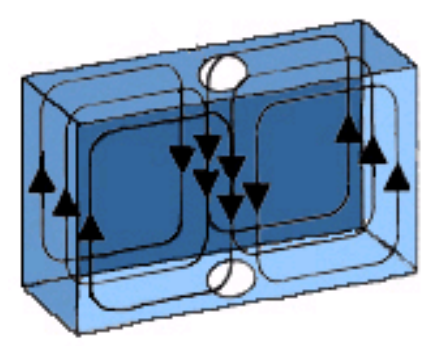

(a)

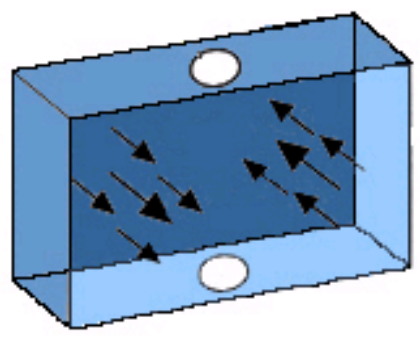

(b)

Figure 2.3. Resonant magnetic field (a) and electric field (b) components of the resonant microwave radiation in a $\mathrm{TE}_{102}$ microwave cavity. (Picture taken from Bruker BioSpin http://www.bruker-biospin.com/cwpractice.html)

microwave source and detector. The microwave source is a Gunn diode.

Figure 2.3 is a diagram of a $\mathrm{TE}_{102}$ mode, rectangular microwave cavity, showing the microwave magnetic and electric fields. The resonance cavity stores microwave energy, and as a result, a standing wave is produced within the cavity. This standing wave has electric and magnetic field components shown in Fig. 2.3. The sample rod is placed down through the center of the cavity (through the top white circle), putting the crystal at the center of the cavity where the microwave magnetic field is maximized and the microwave electric field is minimized (ideally, zero). This placement of the sample minimizes the excitation of electric dipoles within the sample. The cavity used in this study is manufactured with slots in the end that allow for optical access. An Oxford helium-gas-flow system is used to maintain sample temperatures at 4-30 K.

The ENDOR cavity used in this study is a cylindrical $\mathrm{TE}_{011}$ cavity. During an ENDOR experiment, the magnetic field is held at a field at which an EPR line appears. The microwave power is then adjusted in order to optimize the EPR signal (i.e., the microwave power is increased until saturation occurs). Saturation refers to the situation 
where the levels of the spin states are equally populated. In an ENDOR spectrometer, a metal coil inside the cavity encircles the sample. This coil is responsible for applying the rf magnetic field to the crystal. On its own, the rf oscillator provides a field that is too weak to drive these transitions, so an rf amplifier is used to increase the intensity of the rf field. The frequency of the $\mathrm{rf}$ radiation is swept, and NMR transitions are produced at resonant frequencies. These transitions result in the spin level populations being once again unequal, and the EPR signal becomes desaturated. Hence, lines appearing on an ENDOR are changes in the intensity of the EPR absorption.

\subsection{Signal Detection}

EPR employs a technique known as phase-sensitive detection. This is used to increase sensitivity by decreasing noise. Phase-sensitive detection works as follows: the magnetic field at the sample is modulated sinusoidally at a pre-determined modulation frequency, $100 \mathrm{kHz}$ for this study. At an EPR resonance field, the modulated magnetic field sweeps through a portion of the absorption signal that is approximately linear over a user-determined interval known as the modulation amplitude. The modulation amplitude is usually on the order of one gauss. The EPR signal is converted into a sine wave whose amplitude is proportional to the slope of the EPR signal, giving rise to the first-derivative shape of a typical EPR signal. This technique suppresses electrical interference and noise, which leads directly to increased sensitivity.

\subsection{Fourier Transform Infrared Spectrometer}

A Fourier Transform Infrared (FTIR) spectrometer was used in this work to detect 
$\mathrm{OH}$ absorption in $\mathrm{TiO}_{2}$. FTIR works by detecting the absorption of photons by the electric dipole moment of the molecule. In an FTIR experiment, the sample is exposed to an infrared light beam. What separates FTIR methods from older wavelength scanning techniques is the fact that in an FTIR experiment, all incoming frequencies can be measured simultaneously. Conversely, older, "dispersive" instruments could only read the incoming frequencies one at a time. ${ }^{3}$ Figure 2.4 shows a schematic diagram of a Michelson interferometer used for FTIR. The moveable mirror introduces a path difference $\Delta$ between the two split beams. When they recombine, they interfere with one another and form a signal intensity interferogram, which is a function of $\Delta$.

A detector (MCT or DTGS) measures how much light is absorbed by the sample. It does this by comparing the transmitted beam intensity to the intensity of the beam when no sample is present. Vibration modes of defects within a material absorb light at certain characteristic frequencies. These frequencies are governed by the masses of the individual atoms involved and oscillator strength of the transitions. Absorption of certain

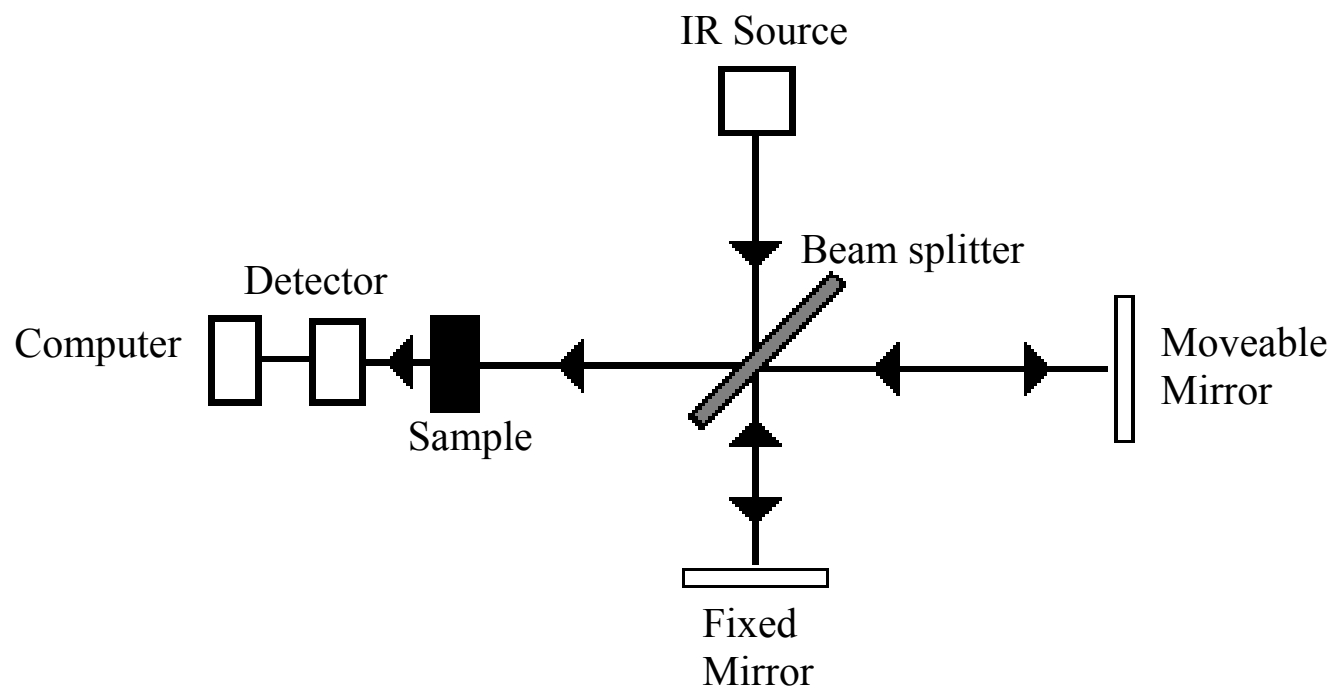

Figure 2.4. Michelson interferometer used for FTIR spectroscopy. The moveable mirror moves in the longitudinal direction (horizontally in this figure) relative to the light beam. 
wavelengths of light causes the molecules to vibrate. The types of vibrations that a molecule can undergo are called modes of vibration. The number of atoms in the molecule and the molecule's linearity determine the number of vibrational modes, or degrees of freedom. A linear molecule has $3 \mathrm{~N}-5$ degrees of freedom while a nonlinear one has $3 \mathrm{~N}-6$.

As mentioned above, only those vibrational modes that result in a change in the dipole moment of the molecule can be detected by FTIR. This is what is meant by the term "IR active". In this study, FTIR is used to detect a stretch mode of an $\mathrm{OH}$ molecule. The electric dipole moment of $\mathrm{N}$ point charges is given by

$$
\vec{p}(\vec{r})=\sum_{i=1}^{N} q_{i}\left(\vec{r}_{i}-\vec{r}\right)
$$

For two opposite point charges, this is simply $\left(\mathrm{q}_{1}+\mathrm{q}_{2}\right) \mathrm{d}$, where $\mathrm{d}$ is the distance between them. For the stretch mode of the $\mathrm{OH}$ molecule, the dipole moment changes as a result of the change in distance between the two atoms.

\subsection{Spin Hamiltonian Analysis}

The spin Hamiltonian describes the contributions to a system's energy from each of the interactions that the unpaired spins experience. In the most general case, the spin Hamiltonian will take the following form ${ }^{4,5}$

$$
\hat{H}=\mu_{\mathrm{B}} \vec{B} \cdot \vec{g} \cdot \vec{S}+\vec{S} \cdot \vec{A} \cdot \vec{I}+\vec{I} \cdot \vec{P} \cdot \vec{I}+\vec{S} \cdot \vec{D} \cdot \vec{S}-g_{n} \mu_{n} \vec{B} \cdot \vec{I}
$$


The terms are defined as follows:

\begin{tabular}{|c|c|l|}
\hline$\mu_{B} \vec{B} \cdot \vec{g} \cdot \vec{S}$ & Electron Zeeman interaction & $\begin{array}{l}\text { Describes the behavior } \\
\text { of a spin in an external } \\
\text { magnetic field }\end{array}$ \\
\hline$\vec{S} \cdot \vec{A} \cdot \vec{I}$ & Hyperfine interaction & $\begin{array}{l}\text { Due to interaction } \\
\text { between an unpaired } \\
\text { spin and a nearby } \\
\text { nucleus }\end{array}$ \\
\hline$\vec{I} \cdot \vec{P} \cdot \vec{I}$ & Nuclear Quadrupole interaction & $\begin{array}{l}\text { Effect of a nucleus with } \\
\text { an electric quadrupole } \\
\text { moment interacting with } \\
\text { an electric field gradient }\end{array}$ \\
\hline$\vec{S} \cdot \vec{D} \cdot \vec{S}$ & Zero-Field interaction & $\begin{array}{l}\text { The result of an } \\
\text { unpaired spin interacting } \\
\text { with an electric crystal } \\
\text { field }\end{array}$ \\
\hline$g_{n} \mu_{n} \vec{B} \cdot \vec{I}$ & Nuclear Zeeman interaction & $\begin{array}{l}\text { Describes the interaction } \\
\text { of a magnetic field with } \\
\text { a nuclear magnetic } \\
\text { moment. }\end{array}$ \\
\hline
\end{tabular}

When solving for the parameters of the spin Hamiltonian, the matrices $\vec{g}, \vec{A}, \vec{P}$, and $\vec{D}$ are transformed into their principal axis systems, so that only six parameters are needed for each matrix; three principal values and three principal axis directions. In general, these matrices are not isotropic. The principal axis directions relate the spin-Hamiltonian parameters to the magnetic field and crystalline coordinate systems. When solving a spin Hamiltonian, all the matrices must be expressed in a common coordinate system. Least squares fitting routines are used in this work to find "best fit" principal values and directions for various spin-Hamiltonian matrices. Two example programs are provided in Appendix A. 
The electron Zeeman interaction contains two parts: one part due to the interaction between a magnetic dipole and an external field and one due to spin-orbit coupling.

$$
\begin{gathered}
\hat{\mathrm{H}}_{\text {dipole }}=\mu_{\mathrm{B}} \overrightarrow{\mathrm{B}} \cdot\left(\overrightarrow{\mathrm{L}}+\mathrm{g}_{\mathrm{e}} \overrightarrow{\mathrm{S}}\right) \\
\hat{H}_{S O}=\lambda \vec{L} \cdot \vec{S}
\end{gathered}
$$

The Hamiltonian is then

$$
\begin{gathered}
\hat{\mathrm{H}}=\hat{\mathrm{H}}_{\text {dipole }}+\hat{\mathrm{H}}_{\mathrm{SO}}=\mu_{\mathrm{B}} \overrightarrow{\mathrm{B}} \cdot\left(\overrightarrow{\mathrm{L}}+\mathrm{g}_{\mathrm{e}} \overrightarrow{\mathrm{S}}\right)+\lambda \vec{L} \cdot \vec{S}=\mu_{\mathrm{B}} \overrightarrow{\mathrm{B}} \cdot \overrightarrow{\mathrm{g}} \cdot \overrightarrow{\mathrm{S}} \\
\vec{g}=g_{e} \overrightarrow{1}+2 \lambda \vec{\Lambda}
\end{gathered}
$$

The tensor $\vec{\Lambda}$ accounts for the deviation of $\mathrm{g}$ from the free electron value and also contributes to the anisotropy of the g matrix.

The hyperfine interaction is generally described by the parameter A. This parameter has two parts, and is written as a matrix of the form

$$
\overleftrightarrow{A}=a \overleftrightarrow{1}+\overleftrightarrow{b}
$$

The parameter a is the isotropic part of the hyperfine matrix and is known as the Fermi contact term. The Fermi contact term is given by

$$
a=\frac{8 \pi}{3} g_{n} \mu_{n} g_{e} \mu_{e}\left|\Psi\left(r_{n}\right)\right|^{2}
$$

$\Psi\left(r_{n}\right)$ is the wavefunction evaluated at the nucleus, and $\mu_{n}$ and $\mu_{\mathrm{e}}$ are the magnetic moments of the electron and nucleus, respectively. The Fermi contact term is only nonzero if there is spin density at the nucleus, i.e., only if there are unpaired electrons in an s orbital. All other orbitals have a node at the origin (i.e., the nuclear site), and hence the Fermi contact term vanishes when unpaired electrons occupy these orbitals. The contact term can be derived from the three principal values of the A matrix by the relation 


$$
a=\frac{\operatorname{Tr}(\vec{A})}{3}=\frac{\left(A_{1}+A_{2}+A_{3}\right)}{3 g_{e} \mu_{\mathrm{B}}} .
$$

It is often said that the hyperfine interaction is the result of magnetic dipole interactions between an electron and a nucleus. The point-dipole approximation, however, is only valid when the electron and nucleus are far away from each other. Classically, the interaction energy between an electron and nuclear magnetic moment is

$$
U_{d i p}(\vec{r})=\frac{-\mu_{0}}{4 \pi}\left[\frac{\vec{\mu}_{e} \cdot \vec{\mu}_{n}}{r^{3}}-\frac{3\left(\vec{\mu}_{e} \cdot \vec{r}\right)\left(\vec{\mu}_{n} \cdot \vec{r}\right)}{r^{5}}\right] .
$$

Here, $r$ is the distance between the electron and nucleus, and $\mu_{\mathrm{e}}$ and $\mu_{\mathrm{n}}$ are the magnetic moments of the electron and nucleus, respectively. Taking $\vec{\mu}_{\mathrm{e}}=-\mathrm{g}_{\mathrm{e}} \mu_{\mathrm{B}} \hat{\mathrm{S}}$ and $\vec{\mu}_{n}=\mathrm{g}_{n} \mu_{n} \hat{\mathrm{I}}$, the interaction energy becomes ${ }^{4,5}$

$$
U_{\text {dip }}(\vec{r})=\frac{\mu_{0} g_{e} \mu_{\mathrm{B}} g_{n} \mu_{n}}{4 \pi}\left[-\frac{\hat{S} \cdot \hat{I}}{r^{3}}+\frac{3(\hat{S} \cdot \vec{r})(\hat{I} \cdot \vec{r})}{r^{5}}\right]
$$

After integrating over the spatial distribution of the electron, one obtains the anisotropic spin Hamiltonian

$$
\begin{aligned}
& \hat{H}_{d i p}=\frac{\mu_{0} g_{e} \mu_{\mathrm{B}} g_{n} \mu_{n}}{4 \pi}\left[\left\langle\frac{1}{r^{3}}-\frac{3 x^{2}}{r^{5}}\right\rangle \hat{S}_{x} \hat{I}_{x}+\left\langle\frac{1}{r^{3}}-\frac{3 y^{2}}{r^{5}}\right\rangle \hat{S}_{y} \hat{I}_{y}+\left\langle\frac{1}{r^{3}}-\frac{3 z^{2}}{r^{5}}\right\rangle \hat{S}_{z} \hat{I}_{z}\right. \\
& \left.-\left\langle\frac{3 x y}{r^{5}}\right\rangle \hat{S}_{x} \hat{I}_{y}-\left\langle\frac{3 y x}{r^{5}}\right\rangle \hat{S}_{y} \hat{I}_{x}-\left\langle\frac{3 x z}{r^{5}}\right\rangle \hat{S}_{x} \hat{I}_{z}-\left\langle\frac{3 z x}{r^{5}}\right\rangle \hat{S}_{z} \hat{I}_{x}-\left\langle\frac{3 y z}{r^{5}}\right\rangle \hat{S}_{y} \hat{I}_{z}-\left\langle\frac{3 z y}{r^{5}}\right\rangle \hat{S}_{z} \hat{I}_{y}\right]
\end{aligned}
$$

This expression can be written in matrix form as follows: 


$$
\begin{aligned}
\hat{H}_{\text {dip }}=\left[\begin{array}{lll}
S_{x} & S_{y} & S_{z}
\end{array}\right] \cdot\left[\begin{array}{ccc}
\left\langle\frac{1}{r^{3}}-\frac{3 x^{2}}{r^{5}}\right\rangle & -\left\langle\frac{3 x y}{r^{5}}\right\rangle & -\left\langle\frac{3 x z}{r^{5}}\right\rangle \\
-\left\langle\frac{3 y x}{r^{5}}\right\rangle & \left\langle\frac{1}{r^{3}}-\frac{3 y^{2}}{r^{5}}\right\rangle & -\left\langle\frac{3 y z}{r^{5}}\right\rangle \\
-\left\langle\frac{3 z x}{r^{5}}\right\rangle & -\left\langle\frac{3 z y}{r^{5}}\right\rangle & \left\langle\frac{1}{r^{3}}-\frac{3 z^{2}}{r^{5}}\right\rangle
\end{array}\right] \cdot\left[\begin{array}{c}
I_{x} \\
I_{y} \\
I_{z}
\end{array}\right] \\
=\hat{S} \cdot \vec{b} \cdot \hat{I}
\end{aligned}
$$

The angular brackets in $\vec{b}$ indicate that the term is averaged over the spatial distribution of the electron. More specifically, the individual elements of $\vec{b}$ are

$$
b_{i j}=\frac{\mu_{0} g_{n} \mu_{n} g_{e} \mu_{e}}{4 \pi} \int \frac{3 x_{i} x_{j}}{r^{5}}-\frac{\delta_{i j}}{r^{3}}|\Psi(r)|^{2} d^{3} r
$$

where $\Psi(\mathrm{r})$ is the electron wavefunction. The tensor $\vec{b}$ accounts for the anisotropy of the hyperfine matrix, and, therefore, the elements of $\vec{b}$ vanish for an s-orbital wavefunction. The nuclear quadrupole interaction comes about when the spin interacts with nuclei that have I $>1 / 2$. These types of nuclei have an electric quadrupole moment that interacts with an electric field gradient (EFG). EFG arises as a result of a non-spherical distribution of charge. ${ }^{6}$ The P matrix often has the same principal axes as the $\mathrm{g}$ and $\mathrm{A}$ matrices. ${ }^{7}$ In its principal axis system, the quadrupole term has the form

$$
\vec{I} \cdot \vec{P} \cdot \vec{I}=P_{x} I_{x}^{2}+P_{y} I_{y}{ }^{2}+P_{z} I_{z}^{2}
$$

Some texts denote the quadrupole parameter as $\mathrm{Q}$. The quadrupole matrix is taken to be traceless, i.e., $\mathrm{P}_{\mathrm{x}}+\mathrm{P}_{\mathrm{y}}+\mathrm{P}_{\mathrm{z}}=0$. From this, the quadrupole term in the Hamiltonian can be written as

$$
\vec{I} \cdot \vec{P} \cdot \vec{I}=\frac{3 P_{z}}{2}\left[\left(I_{z}^{2}-\frac{I(I+1)}{3}\right)+\frac{P_{x}-P_{y}}{P_{z}}\left(I_{x}{ }^{2}-I_{y}{ }^{2}\right)\right]
$$


The coefficient $\frac{P_{x}-P_{y}}{P_{z}}$ is the asymmetry parameter $\eta$, and is a measure of deviation from axial symmetry. The electric field gradient is related to the $\mathrm{z}$ component of the nuclear quadrupole parameter by

$$
P_{z}=\frac{3 e Q}{4 I(2 I-1)} \frac{\partial^{2} V}{\partial z^{2}},
$$

where $\mathrm{Q}$ is the quadrupole moment of the nucleus, and $\frac{\partial^{2} V}{\partial z^{2}}$ is the electric field gradient at the nucleus due to surrounding electrical charges. This quantity is zero for spherical charge distributions.

The contribution from crystalline electric fields is also referred to as a "zero-field splitting" term, as it accounts for separation of energy levels in the absence of an external magnetic field. It can be written in the same form as the nuclear quadrupole term when in the reference frame of the D-matrix, and an asymmetry parameter E can be extracted in an analogous way.

In summary, the spin Hamiltonian describes the different interactions that an unpaired spin (i.e., and electron) experiences. Each of these interactions splits the Zeeman levels into additional levels, which, in turn, leads to additional energy level transitions governed by the EPR selection rules. The result is a complicated EPR spectrum which oftentimes cannot be interpreted without the aid of ENDOR. ENDOR can be used to resolve hyperfine patterns and to definitively assign the defect to specific nucleus. 


\section{Chapter 2 References}

1. N. W. Ashcroft and N. D. Mermin, Solid State Physics (Brooks/Cole, 1976)

2. A. B. Sannigrahi, J. Chem. Educ. 59, 819 (1982).

3. B. C. Smith, Fundamentals of Fourier Transform Infrared Spectroscopy (CRC, Press, Boca Raton, 1996).

4. J.-M. Spaeth, J. R. Niklas, and R. H. Bartram, Structural Analysis of Point Defects in Solids: An Introduction to Multiple Magnetic Resonance Spectroscopy (Springer-Verlag, Heidelberg, 1992).

5. J. A. Weil and J. R. Bolton, Electron Paramagnetic Resonance: Elementary Theory and Practical Applications, 2nd ed. (Wiley, New York, 2007), p. 120.

6. M. J. Duer, Introduction to Solid State NMR Spectroscopy (Blackwell, 2004).

7. A. Abragam and B. Bleaney, Electron Paramagnetic Resonance of Transition Ions (Oxford University Press, New York, 1986). 


\section{Chapter 3}

\section{Hydrogen Donors and $\mathrm{Ti}^{3+}$ Ions}

\subsection{Background}

In 1961, P. F. Chester ${ }^{1}$ published a widely cited paper on electron spin resonance from semiconducting $\mathrm{TiO}_{2}$ (rutile) crystals in which he addressed the point defects appearing in oxygen-deficient (reduced) $\mathrm{TiO}_{2}$. In this work, Chester described a defect called the A center. From the beginning, the discovery of the A center sparked debate as to its origin. There are many researchers who say that the A center is due to a titanium interstitial, ${ }^{2-4}$ and that the interstitial titanium is the dominant shallow donor in $\mathrm{TiO}_{2}$. To quote from Shen et al., "The alternative models of Chester, including that of an unknown center involving hydrogen, were not so much eliminated as ignored." Thus far, no definitive experimental evidence has been reported proving the existence of titanium interstitials in this material. In the present chapter, I show that the A center is a neutral hydrogen donor and suggest that this defect is the most prevalent shallow donor impurity in $\mathrm{TiO}_{2}$. My work suggests that the reduction of $\mathrm{TiO}_{2}$ crystals produces oxygen vacancies, not $\mathrm{Ti}^{3+}$ interstitials.

Chester $^{1}$ gives several possible models for the A center in his original publication, after first mentioning that "a definitive interpretation of [the spectra] cannot be made." His first model assigned the A spectrum to a titanium interstitial, citing the four "open channels" in the unit cell as the likely interstitial sites. This model found favor with researchers because it was a simple and plausible explanation of why the principal axis directions of the g matrix were not along high symmetry directions of the crystal. ${ }^{4}$ Another of Chester's models had the $\mathrm{Ti}^{3+}$ ion at its normal lattice site experiencing a 
perturbation from a nearest-neighbor oxygen vacancy in the basal plane. A third proposal is that the A center is due to the incorporation of hydrogen into the rutile lattice, a model that is favored by the evidence shown in the study described here. Shen et al. ${ }^{5}$ supported the argument for the A center being hydrogen-related by performing EPR studies that showed that the A center did not form in samples that were treated in a manner that was conducive to the formation of $\mathrm{Ti}^{3+}$ interstitials. DeFord and Johnson ${ }^{6}$ determined from calculations that $\mathrm{Ti}^{4+}$ interstitials should form in crystals that are heated in a dry $\mathrm{H}_{2}$ atmosphere, whereas $\mathrm{H}^{+}$concentrations should be higher in samples that are heated in an $\mathrm{H}_{2} \mathrm{O}+\mathrm{O}_{2}$ atmosphere. Shen et al. ${ }^{5}$ supported these predictions when they heated a crystal in a dry-hydrogen atmosphere and found that no hydrogen was incorporated. The A center was not observed in crystals that were treated in this manner. The A center was observed, however, in crystals that were heated in an $\mathrm{H}_{2} \mathrm{O}$ or $\mathrm{D}_{2} \mathrm{O}$ atmosphere. Hydrogen or deuterium was known to have been incorporated into the crystal during these treatments and the A center was observed. This provides strong evidence that the model for the $\mathrm{A}$ center is not interstitial $\mathrm{Ti}^{4+}$ or $\mathrm{Ti}^{3+}$ ions.

In this chapter, I present EPR and ENDOR studies that show that the A center is actually the signature of the neutral hydrogen donor $\mathrm{OH}^{-}$in rutile. Hydrogen exists as $\mathrm{H}^{+}$ (i.e., a proton) and bonds with an oxygen ion in the rutile lattice to form an $\mathrm{OH}^{-}$molecule whose electric dipole axis is oriented perpendicular to the $\mathrm{c}$ axis of the crystal. ${ }^{7-8}$ Free electrons within the crystal are trapped at $\mathrm{Ti}^{4+}$ ions adjacent to $\mathrm{OH}^{-}$ions, thus forming $\mathrm{Ti}^{3+}$ ions. This unpaired electron interacts weakly with the $\mathrm{OH}^{-}$molecule, forming a neutral hydrogen donor defect. The unpaired electron is localized on a $\mathrm{Ti}^{4+}$ ion, and occupies a d orbital. It is not an effective-mass-like wave function, centered at the 
hydrogen defect and spreading out over many lattice spacings.

Principal values and principal axis directions for the $\mathrm{g}$ and hyperfine matrices were obtained from EPR and ENDOR angular dependence studies. I give a model for the electronic structure and defect orientation, and prove, using ENDOR, that the A center is not related to a titanium interstitial. The effect of laser illumination on reduced and unreduced crystals is also examined. Here, "reduced" means that the crystal was placed in an oxygen-deficient environment at high temperature.

\subsection{Samples}

Two reduced single crystals of $\mathrm{TiO}_{2}$ were used in this study. One crystal was purchased from the CrysTec Corporation in Germany. This sample was reduced by heating at $600{ }^{\circ} \mathrm{C}$ for ten minutes in flowing nitrogen gas. The other crystal was purchased from MTI Corporation and was reduced for 50 minutes at $650{ }^{\circ} \mathrm{C}$ in flowing nitrogen gas. As one further reduces a sample, the $\mathrm{Q}$ value of the microwave cavity decreases, meaning that sample is absorbing microwaves nonresonantly. This indicates that reduction increases the number of "free" electrons in the crystal (i.e., raises the Fermi level). I found that these reduction times were sufficient to produce an intense EPR signal from the A center while still maintaining an acceptable $Q$ value in the microwave cavity. These two crystals allowed collection of EPR and ENDOR angular dependence data in all three high-symmetry planes.

\subsection{Infrared Absorption Results}

First, it must be definitively established that hydrogen does indeed exist in these 
crystals. This is done easily with Fourier transform infrared (FTIR) spectroscopy. Figure 3.1 contains two FTIR traces taken from an as-received CrysTec sample, showing an $\mathrm{OH}^{-}$absorption band in a reduced crystal. Figure 3.1 shows an FTIR trace of the $\mathrm{OH}^{-}$ absorption band (a) for a reduced CrysTec sample at room temperature, and (b) for the reduced crystal at $10 \mathrm{~K}$. These crystals were both part of the same as-received boule. The characteristic absorption peak for the $\mathrm{OH}^{-}$vibrational mode ${ }^{9-15}$ in $\mathrm{TiO}_{2}$ is observed at $3277.6 \mathrm{~cm}^{-1}$ at room temperature. At low temperature, the peak shifts to $3282 \mathrm{~cm}^{-1}$ and the linewidth decreases by nearly a factor of 5 . These spectra were taken with unpolarized light that propagated parallel to the $\mathrm{c}$ axis of the crystal. The same absorption band shown in Fig. 3.1 was observed in the crystal before the reduction treatment; it was smaller by a factor of two. This important result makes it clear that hydrogen was introduced to the crystal during the growth process, and not solely during the reduction treatment. The precursor of the A center exists in as-received samples (i.e., the $\mathrm{OH}^{-}$ion),

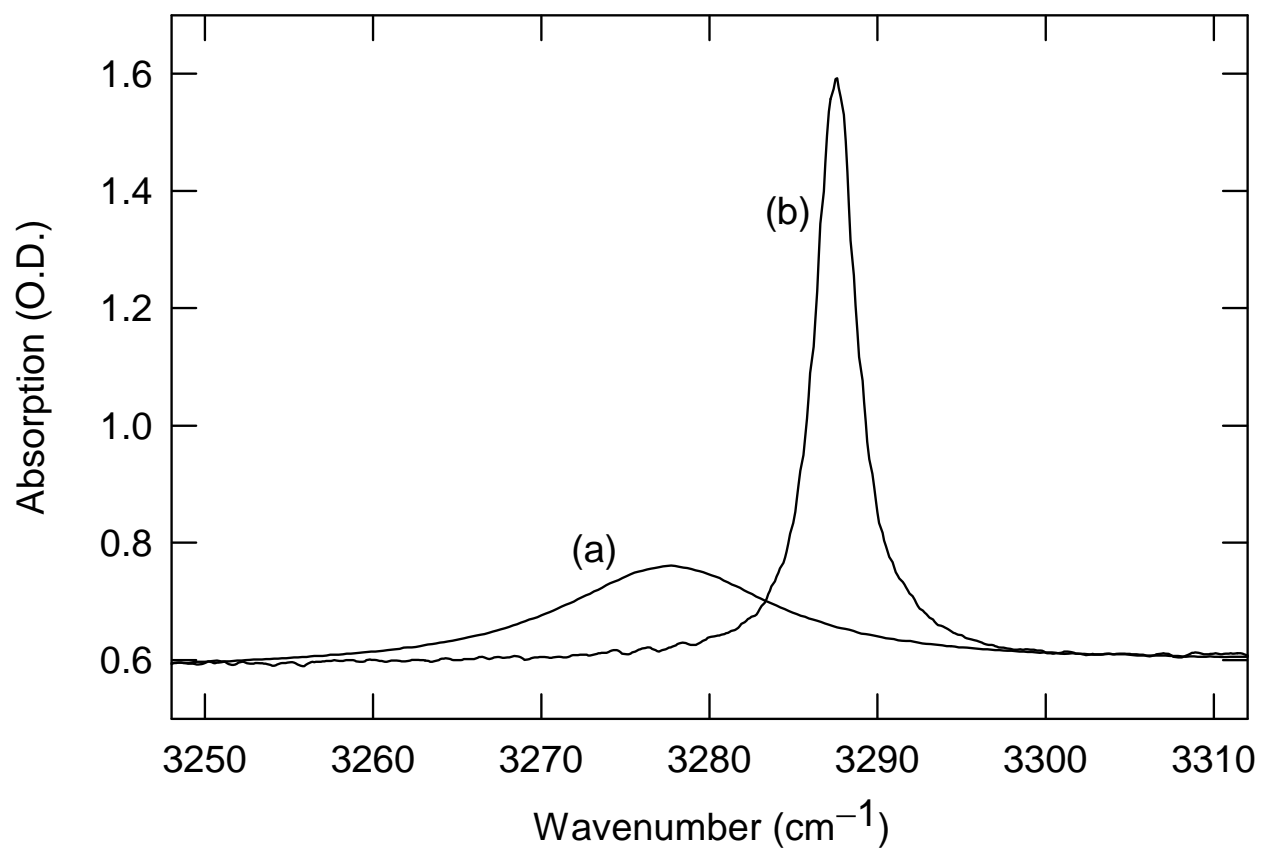

Figure 3.1. FTIR absorption spectra of a reduced $\mathrm{TiO}_{2}$ crystal (a) at room temperature and (b) at $10 \mathrm{~K}$. 
and the EPR spectra associated with the A center are a result of electrons being trapped near the $\mathrm{OH}^{-}$ions. Johnson et al. ${ }^{16}$ derived a method of calculating the concentration of $\mathrm{OH}^{-}$ions in $\mathrm{TiO}_{2}$ (rutile) using the "absorption strength per ion", a parameter related to the oscillator strength. Using their method, I calculated the concentration of $\mathrm{OH}^{-}$ions in Fig 3.1(a) to be $1.45 \times 10^{18} \mathrm{~cm}^{-3}$.

\subsection{EPR Results}

The trapped electron forms an $S=1 / 2$ spin system. The nuclear spin value for hydrogen is $I=1 / 2$, however, the data show only one EPR line. This indicates that the hyperfine interaction is quite weak, resulting in a doublet that is not resolved. Figure 3.2 shows a c-axis spectrum of the hydrogen donor. Figure 3.2(a) was taken from an asgrown CrysTec sample and is produced using $442 \mathrm{~nm}$ light. The trace in 3.2(b) was taken from the reduced sample with no illumination. Each of these spectra was taken at $5 \mathrm{~K}$. These signals broaden and become undetectable when the sample temperature is raised above $10 \mathrm{~K}$. Both of these signals have a c-axis $\mathrm{g}$ value $\left(\mathrm{g}_{[001]}\right)$ of 1.9405 . The hydrogen signal in Fig. 3.2(a) is only visible when $442 \mathrm{~nm}$ laser light is illuminating the sample. The signal completely disappears immediately after the laser is shuttered. The spectrum in Fig. 3.2(b) is not affected by laser illumination.

Figure 3.3 shows the neutral hydrogen donor at three different orientations of the magnetic field in the basal plane. One can see from this that there is not a resolved hyperfine pattern for any orientation of magnetic field. The splittings observed in these spectra are due to site splitting. Site splitting occurs when there are multiple, magnetically inequivalent orientations of the defect. The site splitting observed in 


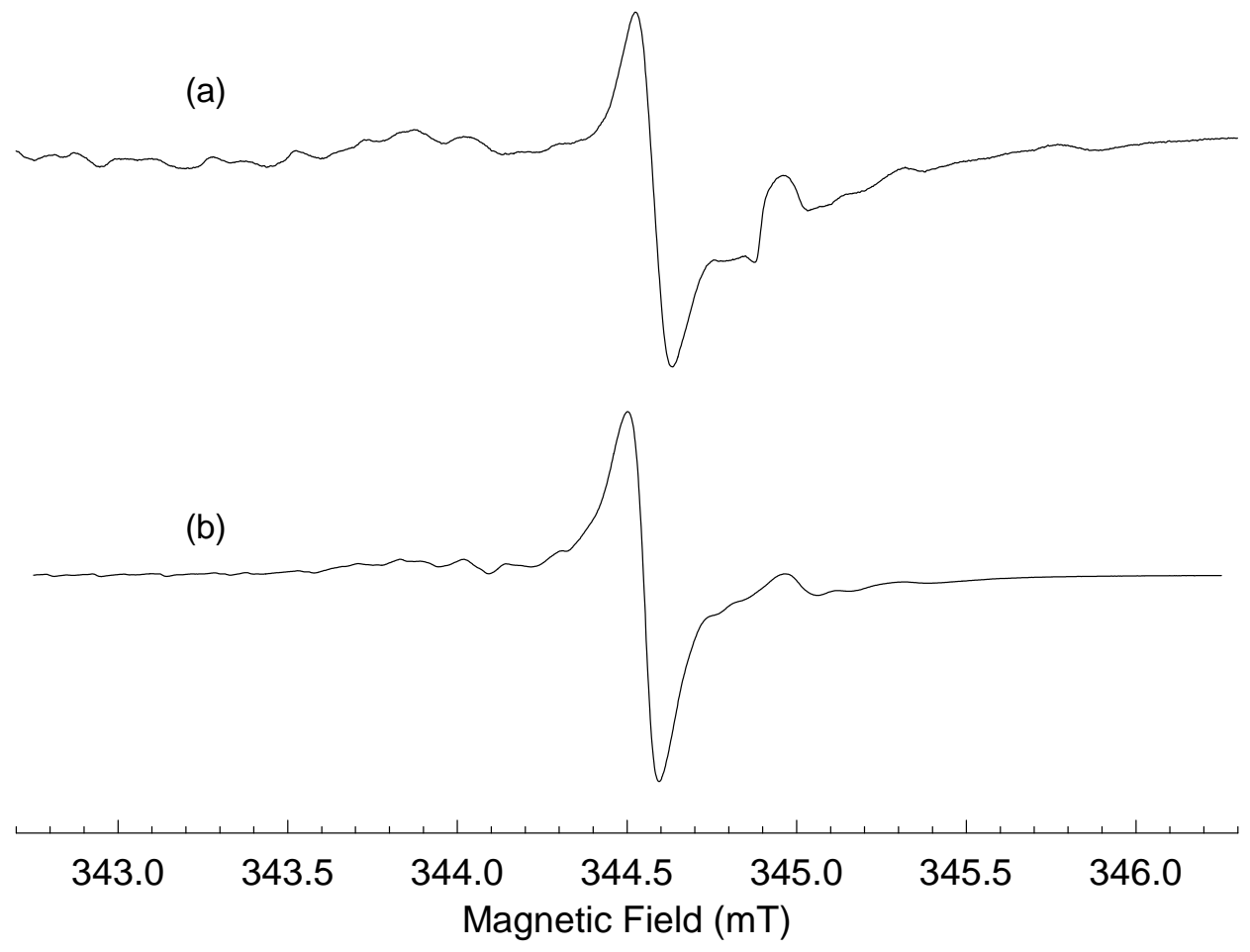

Figure 3.2. EPR spectrum of the neutral hydrogen donor taken at $5 \mathrm{~K}$ with the magnetic field along the [001] crystal axis. These spectra were taken from (a) an as-received sample illuminated with $442 \mathrm{~nm}$ laser light and (b) the reduced CrysTec sample with no laser light. The signal near $345 \mathrm{mT}$ is due to a different, unidentified defect in the crystal. These spectra were taken at $9.371892 \mathrm{GHz}$.

Fig. 3.3 provides evidence as to how the defect is oriented in the crystal. The four lines observed when the magnetic field is in the basal plane indicates that there are four magnetically inequivalent orientations of the defect, which is consistent with a defect located in an interstitial position.

Figure 3.2 shows that the A center can be seen in an as-received crystal illuminated with $442 \mathrm{~nm}$ light and without light in a reduced crystal. I propose the following explanation of this observation. Oxygen ions in a perfect rutile lattice exist in the $\mathrm{O}^{2-}$ charge state. Hydrogen ions $\left(\mathrm{H}^{+}\right)$covalently bond with oxygen to form $\mathrm{OH}^{-}$ molecules. Illumination creates electron-hole pairs and at low temperature, an electron is trapped at a titanium site near an $\mathrm{OH}^{-}$molecule, forming a neutral hydrogen-donor 

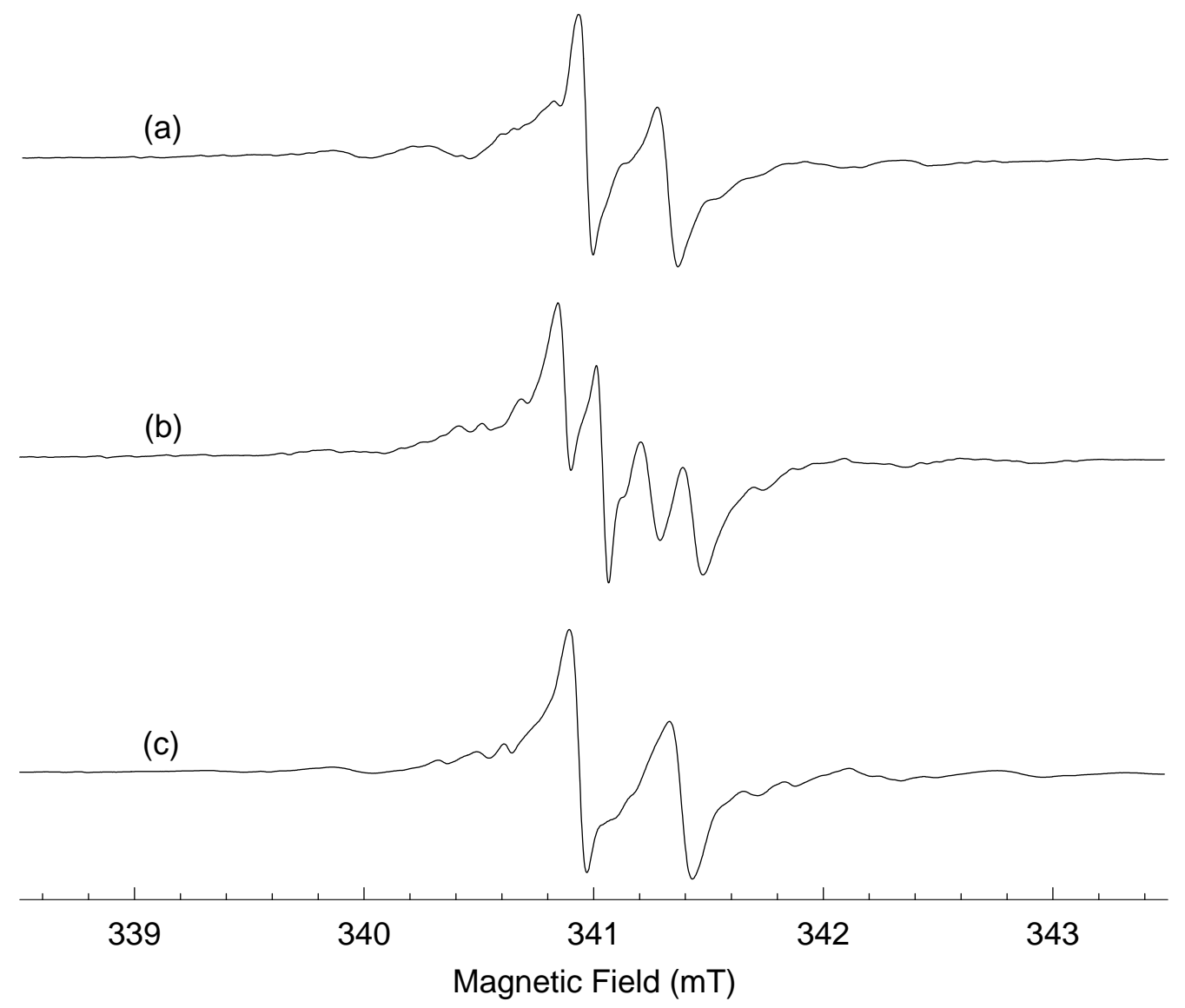

Figure 3.3. EPR spectrum of the neutral hydrogen donor taken with the magnetic field aligned along (a) the [100] crystal axis, (b) $30^{\circ}$ from the [100] axis, and (c) the [110] axis. Four magnetically inequivalent sites are easily seen. These spectra were taken at $5 \mathrm{~K}$.

center. Reduction, on the other hand, creates a greater concentration of oxygen vacancies in the crystal, thereby raising the Fermi level. More "free electrons" are present in the crystal and are trapped at available electron traps when the sample temperature is lowered to $5 \mathrm{~K}$. One can conclude from this that illumination and reduction produce a similar effect; each method creates conduction-band electrons that are trapped at $\mathrm{Ti}^{4+}$ sites at low temperature and interact with nearby $\mathrm{OH}^{-}$molecules. 


\subsection{ENDOR Results}

Figures 3.2 and 3.3 do not give direct evidence that the observed signals are associated with hydrogen. The weak hyperfine interaction does not produce an EPR doublet that one would expect for an $S=1 / 2, I=1 / 2$ spin system; in principle, one would expect two lines from such a system. ENDOR experiments are needed to measure the hyperfine splitting and also to definitively identify the signal as hydrogen-related. There were no conditions under which the hyperfine splitting was resolved with EPR.

Figure 3.4 shows a c-axis ENDOR spectrum of the neutral hydrogen donor. Since the hyperfine interaction is weak, i.e., $\mathrm{A} / 2<v_{\mathrm{n}}$, the two ENDOR lines are centered on $v_{\mathrm{n}}$ and separated by A. Figure 3.4 was obtained by fixing the magnetic field at the EPR resonance magnetic field, $3492.46 \mathrm{G}$. At this field, the known value of $v_{\mathrm{n}}$ for hydrogen is 14.870 MHz. The two large ENDOR transitions in Fig. 3.4 occur at $14.713 \mathrm{MHz}$ and 15.055 MHz. The center of these two lines is at $14.884 \mathrm{MHz}$, in good agreement with the known value of $v_{\mathrm{n}}$. This proves that Chester's A center is a neutral hydrogen donor. The separation of these two ENDOR lines gives a hyperfine parameter of $0.338 \mathrm{MHz}$ in this direction. The center line in Fig. 3.4, at 14.884 MHz, appears at $v_{\mathrm{n}}$, and is due to the unpaired spin interacting with distant hydrogen nuclei in the crystal. The transition at 14.55 MHz is likely paired with another barely observed signal around $15.2 \mathrm{MHz}$ and is due to an unidentified hydrogen-related defect.

Additional ENDOR data were taken with the magnetic field oriented in the basal plane. Figures 3.5 and 3.6 show ENDOR data taken with the magnetic field aligned along the [110] and [110] directions, respectively. Figure 3.5(a) was taken with the magnetic field fixed on the low-field EPR line, which was at 3399.97 G. The two 


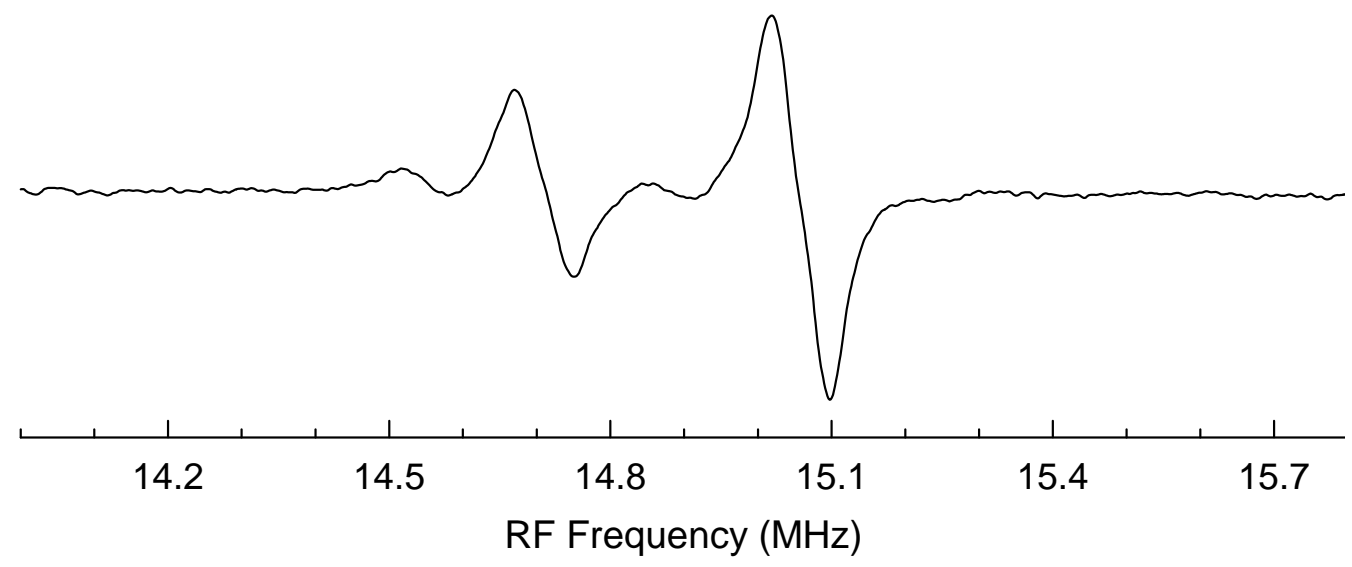

Figure 3.4. ENDOR spectrum of the neutral hydrogen donor taken with the magnetic field along the [001] axis. This spectrum was taken at $5 \mathrm{~K}$.

ENDOR lines are centered at $14.475 \mathrm{MHz}$. The value of $v_{\mathrm{n}}$ at this magnetic field is 14.476 MHz. Trace 3.5(b) was taken with the field fixed on the high-field EPR line, 3402.74 G. These two lines are centered at 14.489 MHz. At this field, the value of $v_{\mathrm{n}}$ is 14.487 MHz. The two lines in trace 3.6(a) are centered at 14.513 MHz, and were taken

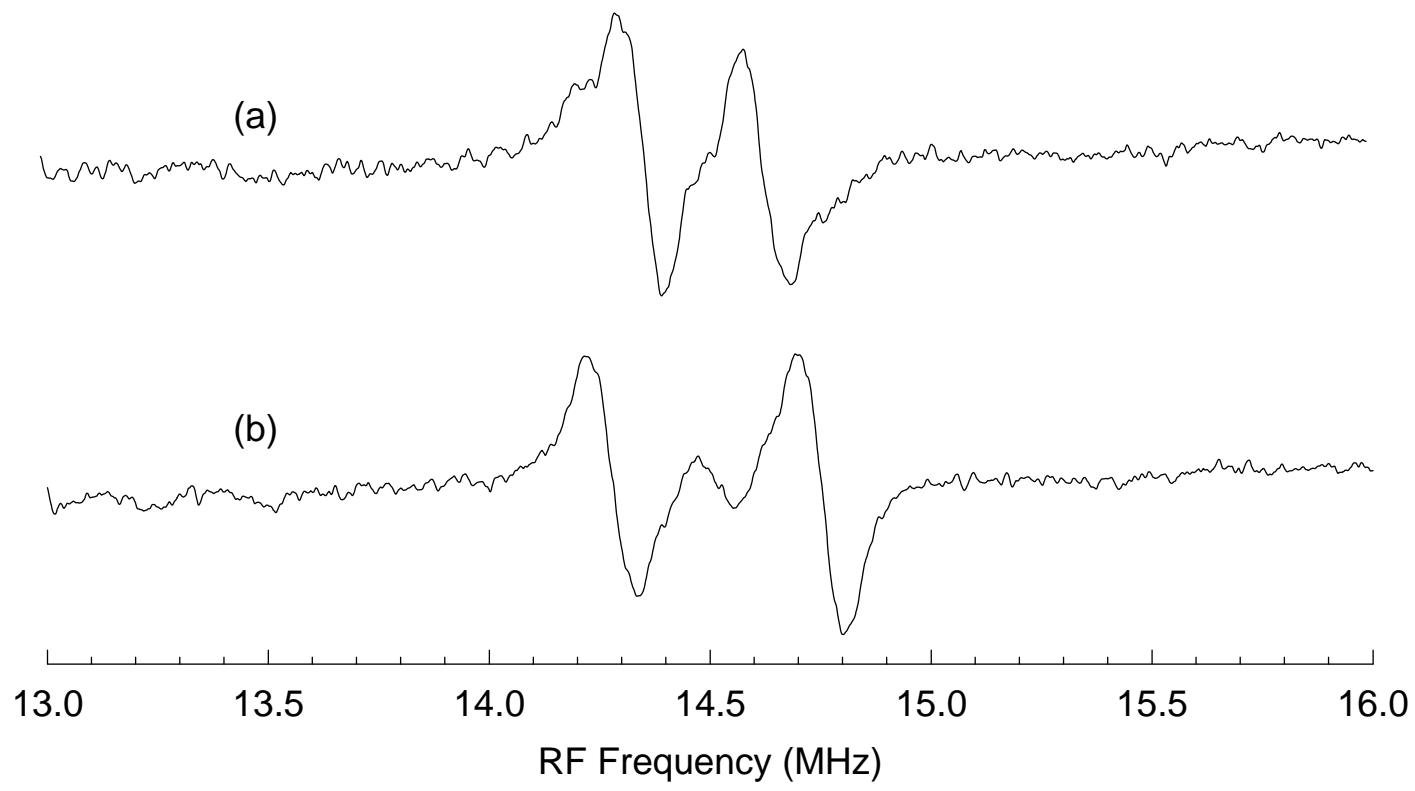

Figure 3.5. ENDOR spectrum of the neutral hydrogen donor taken with the magnetic field aligned along the [100] axis. This spectrum was taken at $5 \mathrm{~K}$. Trace (a) was taken with the magnetic field fixed on the low-field EPR line and trace (b) was taken with the field fixed on the high-field EPR line. 


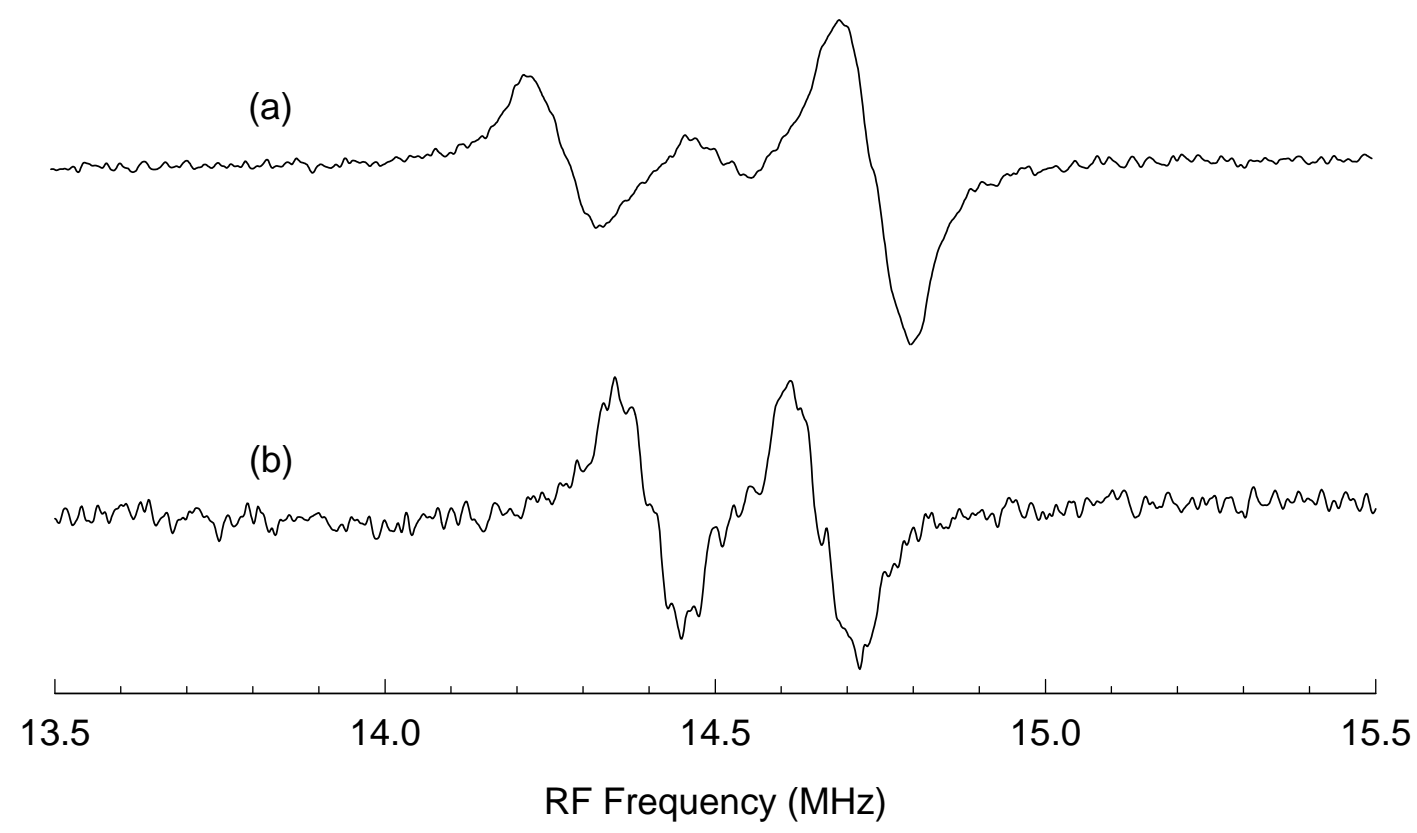

Figure 3.6. ENDOR spectrum of the neutral hydrogen donor taken with the magnetic field aligned along the [110] axis. This spectrum was taken at $5 \mathrm{~K}$. Trace (a) was taken with the magnetic field fixed on the low-field EPR line and trace (b) was taken with the field fixed on the high-field EPR line.

with the magnetic field fixed at $3406.71 \mathrm{G}$. At this field, $v_{\mathrm{n}}=14.504 \mathrm{MHz}$. The two lines in trace 3.6(b) are centered at 14.524 MHz. Here, the field was fixed at $3411.07 \mathrm{G}$, at which $v_{\mathrm{n}}=14.523 \mathrm{MHz}$. The pairs of ENDOR lines in all four of these traces are centered at valued that are very close to $v_{n}$, providing more direct evidence of the identity of the A center. Comparing traces (a) and (b) in both Figs. 3.5 and 3.6, one can see that the lines have different separations, showing the different hyperfine interactions at the two magnetically inequivalent sites of the defect.

\subsection{Spin Hamiltonian Analysis}

In order to determine the principal values and principal axis directions for the $g$ and hyperfine matrices, complete sets of angular dependence data of the EPR and 


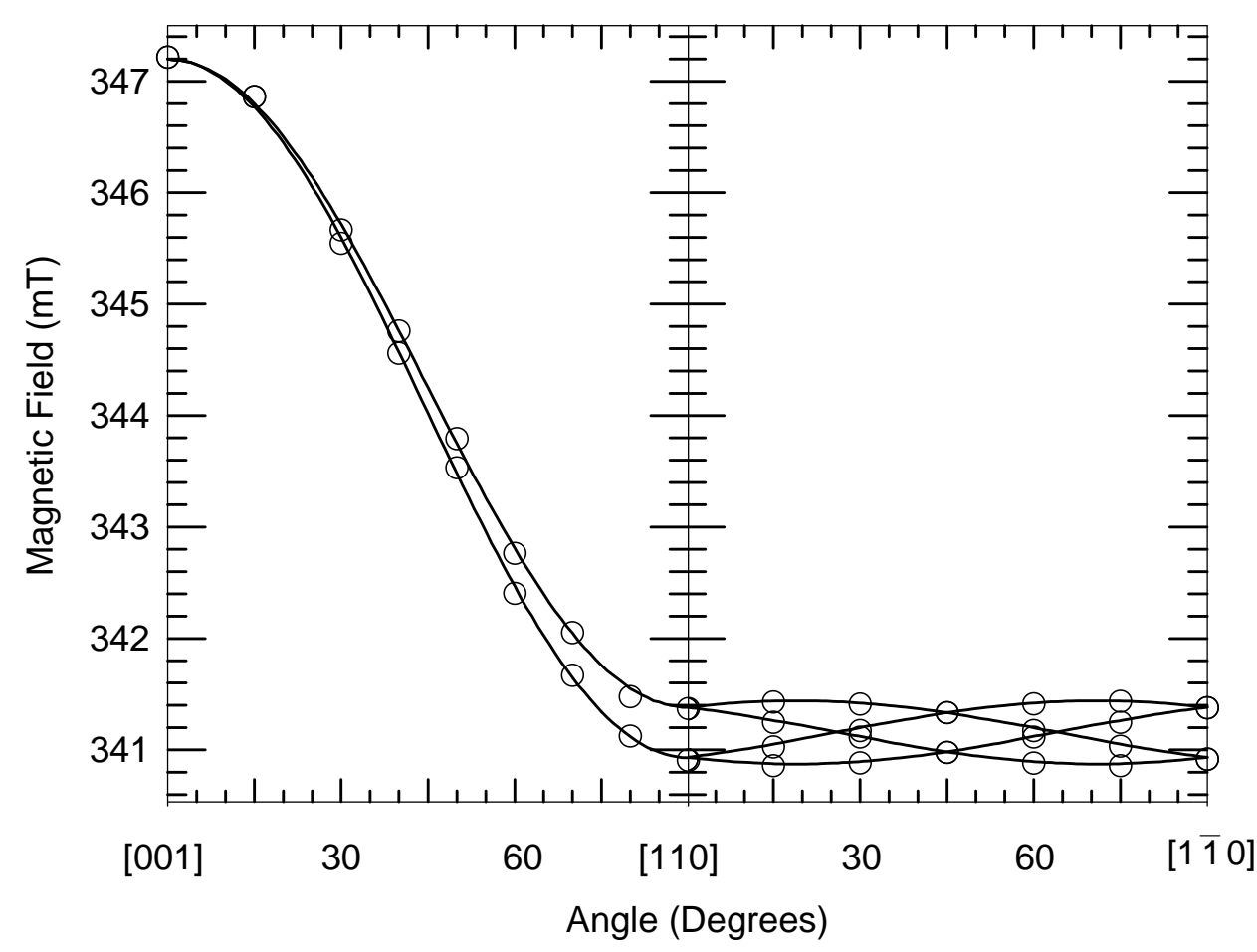

Figure 3.7. Angular dependence of the neutral hydrogen donor EPR signal. The circles represent raw data while the solid lines represent "best fit" lines, generated using the $\mathrm{g}$ values determined by the $\mathrm{g}$ fitting routine in Appendix A. Data were taken at $5 \mathrm{~K}$ at a microwave frequency of $9.429728 \mathrm{GHz}$.

ENDOR signals were obtained and are shown in Figs. 3.7 and 3.8. The $g$ and A values were then extracted from these data by diagonalizing the spin- Hamiltonian matrix using a least squares fitting routine. The routines for fitting both $\mathrm{g}$ and $\mathrm{A}$ are given in Appendices A.1 and A.2, respectively. EPR angular dependence data were obtained using the reduced CrysTec sample. Figure 3.7 shows the angular dependence of the neutral hydrogen donor when the magnetic field is rotated in the $[1 \overline{1} 0]$ and basal planes.

The following spin Hamiltonian was used to calculate the principal values and principal axis directions for the g matrix.

$$
\hat{\mathrm{H}}=\mu_{\mathrm{B}} \vec{S} \cdot \vec{g} \cdot \vec{B}
$$

It was known initially that one of the principal axis directions was along the $\mathrm{c}$ axis. This 
then requires the other two principal axis directions to lie in the basal plane of the crystal. This assignment of a principal axis to the [001] direction can be made because the c-axis g value is an extremum, i.e., the [001] axis is a high-field turning point of the EPR signal when the magnetic field is rotated. With this observation, only two planes of EPR angular dependence data are necessary.

Angular dependence of the hydrogen ENDOR signals were used to determine the principal axis values and directions of the hyperfine matrix. Figure 3.8 shows the ENDOR angular dependence in the three high symmetry planes. The raw data in the second and third panels of Fig. 3.8 were taken from the CrysTec sample. In this sample, no ENDOR signals were observed when the field was aligned along the c-axis, or when within 40 degrees of the $\mathrm{c}$ axis. This is in contrast to the MTI sample used for the first

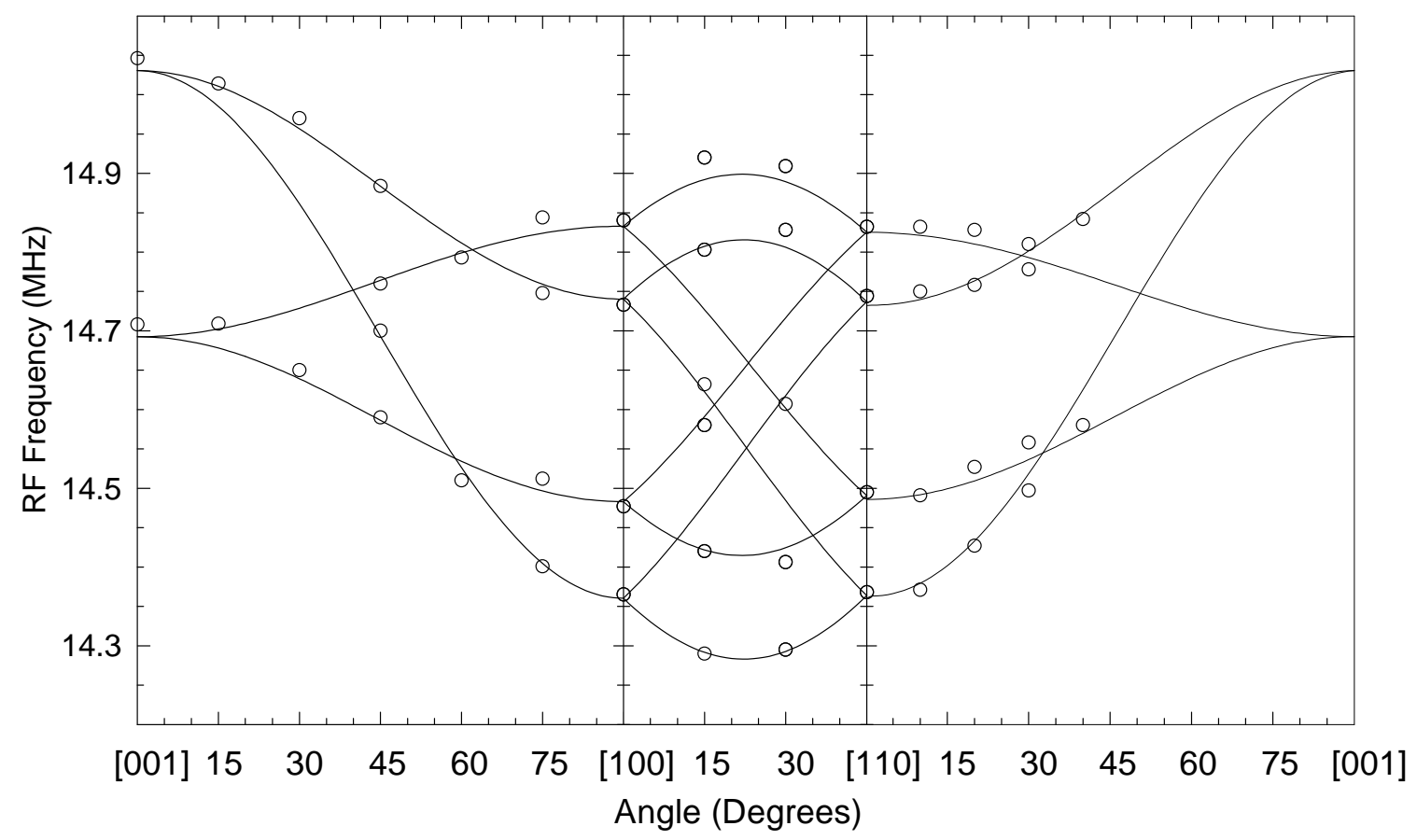

Figure 3.8. Angular dependence of the neutral hydrogen donor ENDOR signal. The circles represent experimental data while the solid lines represent "best fit" lines, generated using the $\mathrm{g}$ and A values determined by the routines in Appendices A.1 and A.2. Data were taken at $5 \mathrm{~K}$ and at a microwave frequency of $9.480087 \mathrm{GHz}$. 
panel. In that sample, ENDOR signals were easily observed when the magnetic field was along the $\mathrm{c}$ axis. A possible explanation for this is that the spin-lattice relaxation times for this defect are different in the two samples when the field is aligned along the [001] axis. It was reported by Yang and Halliburton ${ }^{19}$ that MTI crystals contain substitutional fluorine defects. Yang ${ }^{22}$ also reported that these crystals produce EPR signals that he attributed to interstitial sodium ions. These additional defects were not observed in CrysTec samples. These defects could have an effect on the spin-lattice relaxation times of electrons in $\mathrm{TiO}_{2}$ (rutile).

The following spin Hamiltonian was used to determine the hyperfine parameters:

$$
\hat{\mathrm{H}}=\mu_{\mathrm{B}} \vec{S} \cdot \vec{g} \cdot \vec{B}+\vec{I} \cdot \vec{A} \cdot \vec{S}-g_{n} \mu_{n} \vec{I} \cdot \vec{B}
$$

Table 3.1. Principal values and principal axis directions of the $\mathrm{g}$ and A matrices for the neutral hydrogen donor in $\mathrm{TiO}_{2}$ (rutile).

$\begin{array}{ll}\text { Principal Values } & \begin{array}{l}\text { Principal Axis } \\ \text { Directions }\end{array}\end{array}$

$\begin{array}{ccc}\text { g matrix } & \pm 0.0002 & \\ \mathrm{~g}_{1} & 1.9736 & 18.9^{\circ} \text { from }[1 \overline{1} 0] \\ \mathrm{g}_{2} & 1.9765 & 18.9^{\circ} \text { from }[110] \\ \mathrm{g}_{3} & 1.9405 & {[001]}\end{array}$

A matrix $\quad \pm 0.01 \mathrm{MHz}$

$\begin{array}{lll}\mathrm{A}_{1} & -0.401 \mathrm{MHz} & 22.9^{\circ} \text { from }[1 \overline{1} 0] \\ \mathrm{A}_{2} & 0.616 \mathrm{MHz} & 22.9^{\circ} \text { from }[110] \\ \mathrm{A}_{3} & -0.338 \mathrm{MHz} & \end{array}$


Table 3.1 gives the principal values and principal axis directions of the $g$ and hyperfine matrices. These results were obtained using the fitting routines in Appendix A. Absolute signs of the hyperfine parameters cannot be determined, but relative signs can be determined. The signs given in Table 3.1 were chosen in order to make the dipole-dipole contribution to the A matrix positive.

\subsection{Defect Model}

From Fig. 3.2 alone, the model of an interstitial hydrogen atom can be ruled out immediately. If that model were true, a two-line, nearly isotropic spectrum with $\sim 506$ Gauss separation between the two lines would be observed. ${ }^{17}$ Reference 17 reports that the isotropic hyperfine constant for hydrogen is $50.685 \mathrm{mT}$. The isotropic part is the only contribution to the hyperfine matrix since the hydrogen atom's electron is in an s orbital. The absorption band in Figure 3.1 is consistent with several other works that report the basal-plane orientation of the $\mathrm{OH}^{-}$electric dipole.

Figures 3.2 and 3.3 lend insight into the different orientations of the $\mathrm{OH}^{-}$ion. Figures 3.3(a) and 3.3(c) show two distinct EPR lines when the field is oriented along high symmetry directions in the basal plane. Figure 3.3(b) shows four resolved EPR lines for an in-between orientation in the basal plane. This indicates that there are four distinguishable orientations for the $\mathrm{OH}^{-}$defect. When oriented in the [1 10$]$ or [010] planes, there are two magnetically inequivalent sites. In between, there are four. For some researchers, this four-fold multiplicity justified the existence of a $\mathrm{Ti}^{3+}$ interstitial, when, in fact, an $\mathrm{OH}^{-}$molecule arranged as shown in Fig. 3.9 gives similar angular behavior. Figure 3.3 is quite similar to the angular behavior observed by Chester. ${ }^{1}$ 
Figure 3.9 depicts a basal plane-view of $\mathrm{a} \mathrm{Ti}^{3+}$ ion and the two oxygen ions that are located along the elongated direction of the $\mathrm{TiO}_{6}$ octahedron. The figure illustrates four possible sites (orientations) that the hydrogen ion could occupy in rutile. It does not mean that there is an $\mathrm{OH}^{-}$molecule at each of the four sites simultaneously. Four additional sites are oriented similarly to the neighboring $\mathrm{TiO}_{6}$ octahedron, which is rotated $90^{\circ}$ relative to the one shown in Fig. 3.9. Those four sites, however, are magnetically equivalent to the sites depicted in Fig. 3.9, and do not contribute additional EPR lines. The assignment of sites 1 through 4 is arbitrary, but one can determine which are equivalent in certain orientations by examining a rutile crystal model or Fig. 3.9. One can see that for field alignment along the [110] direction, sites 1 and 2 are magnetically equivalent, as are sites 3 and 4. For field alignment along the [100] direction, sites 1 and 3 are equivalent, as are sites 2 and 4 . The basal plane angular dependence agrees with this model.

In the absence of lattice relaxation, the bonds between $\mathrm{Ti}^{3+}$ and $\mathrm{O}^{2-}$ and between $\mathrm{O}^{2-}$ and $\mathrm{H}^{+}$form a right triangle. From the known $\mathrm{Ti}^{3+}-\mathrm{O}^{2-}$ and $\mathrm{OH}^{-}$bond lengths, ${ }^{18}(1.98$ $\AA$ and $0.96 \AA$, respectively), one calculates the $\mathrm{H}^{+}$ion to lie $25.86^{\circ}$ from the [110] axis. This is an important piece of information. The principal axis directions of the $g$ and hyperfine matrices were 18.9 and $22.9^{\circ}$, which are both close to the angle between the [110] axis and the line joining $\mathrm{Ti}^{3+}$ and $\mathrm{H}^{+}$. The largest hyperfine parameter, $\mathrm{A}_{2}=0.616$ $\mathrm{MHz}$, is assigned to the direction pointing closest to the $\mathrm{H}^{+}$ion. This clearly establishes the model of a neutral hydrogen donor as depicted in Fig. 3.9. 


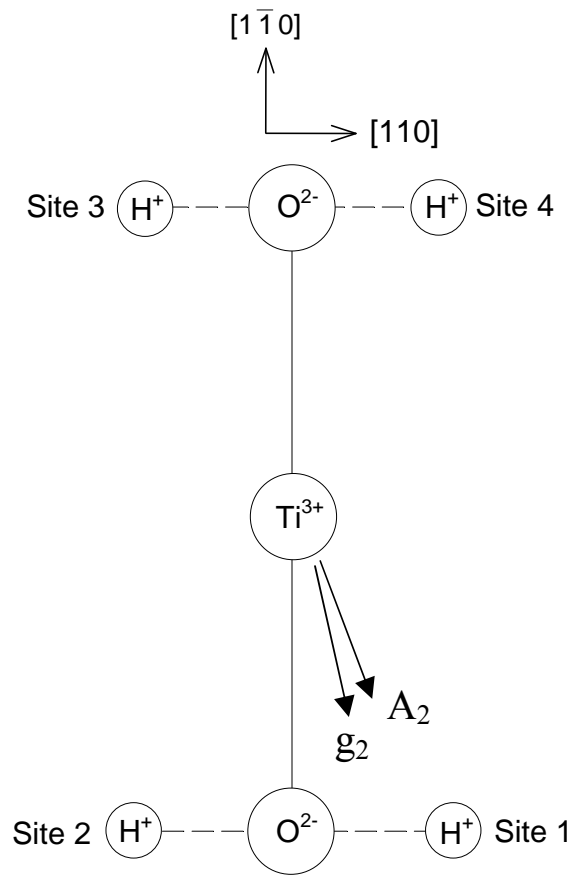

Figure 3.9. Schematic diagram of $\mathrm{OH}^{-}$ions in rutile. The two oxygen ions shown are the two along the elongated axis of the $\mathrm{TiO}_{6}$ octahedron. The principal axis directions of $\mathrm{g}_{2}$ and $\mathrm{A}_{2}$ are also shown. They lie $18.9^{\circ}$ and $22.9^{\circ}$ degrees from the [110] direction, respectively.

\subsection{Further Analysis of the Hyperfine Matrix}

As discussed in Chapter 2, the hyperfine matrix can be written as

$$
\vec{A}=a \overrightarrow{1}+\vec{b}
$$

The elements of $\vec{b}$ are all zero when the unpaired spin occupies an s-orbital. The hyperfine matrix remains isotropic due to the spherical symmetry of the s orbitals. In matrix notation, Eq. 1 can be written as follows

$$
\left(\begin{array}{ccc}
A_{1} & 0 & 0 \\
0 & A_{2} & 0 \\
0 & 0 & A_{3}
\end{array}\right)=\left(\begin{array}{lll}
a & 0 & 0 \\
0 & a & 0 \\
0 & 0 & a
\end{array}\right)+\left(\begin{array}{ccc}
-b+b^{\prime} & 0 & 0 \\
0 & 2 b & 0 \\
0 & 0 & -b-b^{\prime}
\end{array}\right)
$$

The parameters $b$ and $b^{\prime}$ are anisotropic hyperfine interaction constants. The parameter $b^{\prime}$ is a measure of the amount the matrix deviates from axial symmetry. The parameter $b$ 
indicates how the unpaired electron spin is distributed. The anisotropic part of the hyperfine matrix arises from the dipolar interaction between the unpaired electron and the nearby nucleus. Using the principal values of A from Table 3.1, a, b, and b' turn out to be

$$
\begin{gathered}
\mathrm{a}=-0.041 \mathrm{MHz} \\
\mathrm{b}=0.3285 \mathrm{MHz} \\
\mathrm{b}^{\prime}=-0.0315 \mathrm{MHz} .
\end{gathered}
$$

We see from this calculation that the anisotropic portion of $\mathrm{A}$ is nonzero, meaning that the unpaired spin does not solely occupy an s-orbital. One can also show that the electron and nucleus do not have a classical point dipole interaction. In Chapter 2, the elements of $\vec{b}$ were shown to be

$$
b_{i j}=\frac{\mu_{0} g_{n} \mu_{n} g_{e} \mu_{e}}{4 \pi} \int \frac{3 x_{i} x_{j}}{r^{5}}-\frac{\delta_{i j}}{r^{3}}|\Psi(r)|^{2} d^{3} r .
$$

In a point dipole approximation, $\psi(\mathrm{r})$ becomes a Dirac delta function, $\delta(\mathrm{r})$. Equation 2 then reduces to

$$
b=\frac{\mu_{0} g \mu_{\mathrm{B}} g_{n} \mu_{n}}{4 \pi R^{3}} .
$$

The variable $\mathrm{R}$ is the distance between the unpaired spin and the interacting nucleus. It has been established that the unpaired spin is trapped at a titanium site with an adjacent $\mathrm{OH}^{-}$molecule. Therefore, I will assume that $\mathrm{R} \approx 1 \AA$ A. Equation 2a then gives $\mathrm{b}=534.06$ MHz., in strong disagreement with the experimentally determined value of $b$. This exercise shows that the interaction between the unpaired electron and the hydrogen nucleus is not a simple dipole-dipole interaction and that the unpaired spin is not in a hydrogenic s orbital. This gives further support to my conclusion that the unpaired spin is trapped near a titanium ion and occupies a d orbital. 


\section{9 d Orbital Energy Levels}

The unpaired electron is localized on a titanium ion near the $(\mathrm{OH})^{-}$defect, and is in the $3 \mathrm{~d}^{1}$ configuration. Using the experimentally determined $\mathrm{g}$ values, one can determine the energy level scheme of the $\mathrm{d}$ orbitals and determine the ground state orbital. This analysis was done previously in a study by Yang and Halliburton ${ }^{19}$ on fluorine donors in rutile. The principal $g$ values for that defect turned out to be very similar to those of the neutral hydrogen donor.

Principal g values differ from the g value of a "free electron" $\left(g_{e}=2.0023\right)$ due to spin-orbit interaction. The effective $\mathrm{g}$ value is the given by

$$
\vec{g}=g_{e} \overrightarrow{1}+2 \lambda \vec{\Lambda}
$$

with the elements of $\hat{\Lambda}$ given by

$$
\Lambda_{i j}=-\sum_{n \neq G} \frac{\left\langle G\left|\hat{L}_{i}\right| n\right\rangle\left\langle n\left|\hat{L}_{j}\right| G\right\rangle}{E_{n}^{(0)}-E_{G}^{(0)}}
$$

$|G\rangle$ is the ground level, and $|n\rangle$ are the higher levels. This additional term lifts the degeneracy of the $d$ orbitals and is the cause of the anisotropic nature of the $g$ matrix. $\lambda$ is the Russell-Saunders parameter, which is the same as the spin-orbit coupling constant for $\mathrm{S}=1 / 2$ systems. $^{20}$

Figure 3.10 is a diagram of the rutile unit cell and it defines the coordinate system that will be used to determine the ground state $\mathrm{d}$ orbital. The coordinate system is oriented in such a way that the three axes correspond to the three principal axis directions of the g matrix. This was done so that the principal $g$ values can be used in equation 3 . Figure 3.11 below shows the configuration of the five d orbitals. In crystal field theory, the transition metal ion and surrounding ligands are treated as point ions. The bonds 


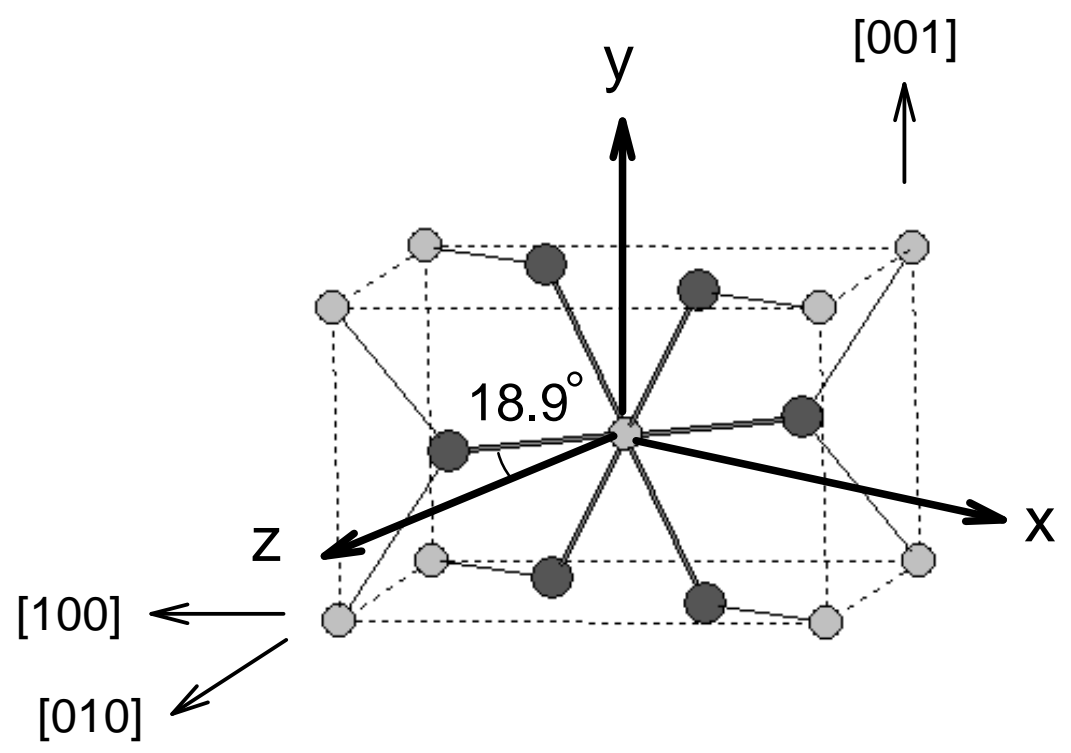

Figure 3.10. Unit cell of rutile, defining the Cartesian coordinate system that is used to determine the d-orbital energy levels. The $\mathrm{z}$ and $\mathrm{x}$ axes lie in the basal plane, and the system is rotated $18.9^{\circ}$ away from the [110] axis.

between the two are not considered. One can see from the previous two figures that the $\mathrm{d}_{\mathrm{yz}}, \mathrm{d}_{\mathrm{xz}}$ and $\mathrm{d}_{\mathrm{x}-\mathrm{y}}^{2}{ }^{2}$ orbitals lie farther away from the negative point ions (oxygen ions) than do the $\mathrm{d}_{\mathrm{xy}}$ and $\mathrm{d}_{\mathrm{z}}{ }^{2}$ orbitals. This means that it would be more energetically favorable for the electron to occupy the $\mathrm{d}_{\mathrm{yz}}, \mathrm{d}_{\mathrm{xz}}$ and $\mathrm{d}_{\mathrm{x}-\mathrm{y}}{ }^{2}$ orbitals than the $\mathrm{d}_{\mathrm{xy}}$ and $\mathrm{d}_{\mathrm{z}}{ }^{2}$ orbitals. By symmetry, the $\mathrm{d}_{\mathrm{yz}}, \mathrm{d}_{\mathrm{xz}}$ and $\mathrm{d}_{\mathrm{x}-\mathrm{y}}{ }^{2}{ }^{2}$ orbitals form a degenerate set (referred to as $\mathrm{t}_{2 \mathrm{~g}}$, which represents a triplet set), as do the $\mathrm{d}_{\mathrm{xy}}$ and $\mathrm{d}_{\mathrm{z}}{ }^{2}$ orbitals ( $\mathrm{e}_{\mathrm{g}}$, denoting a doublet set). The $\mathrm{t}_{2 \mathrm{~g}}$ and $\mathrm{e}_{\mathrm{g}}$ sets are separated by the parameter $\Delta$.

Distortions of the lattice further remove the degeneracy of these two sets. The four oxygen ions in the [110] plane of rutile are not arranged squarely around the [110] axis. Therefore, splitting occurs in the $t_{2 g}$ set, resulting in the $\mathrm{d}_{\mathrm{x}-\mathrm{y}}{ }^{2}$ level having the lowest energy. The levels are separated by $\delta_{1}$ and $\delta_{2}$. The arrangement of the energy levels will be shown using Eq. 3 . 

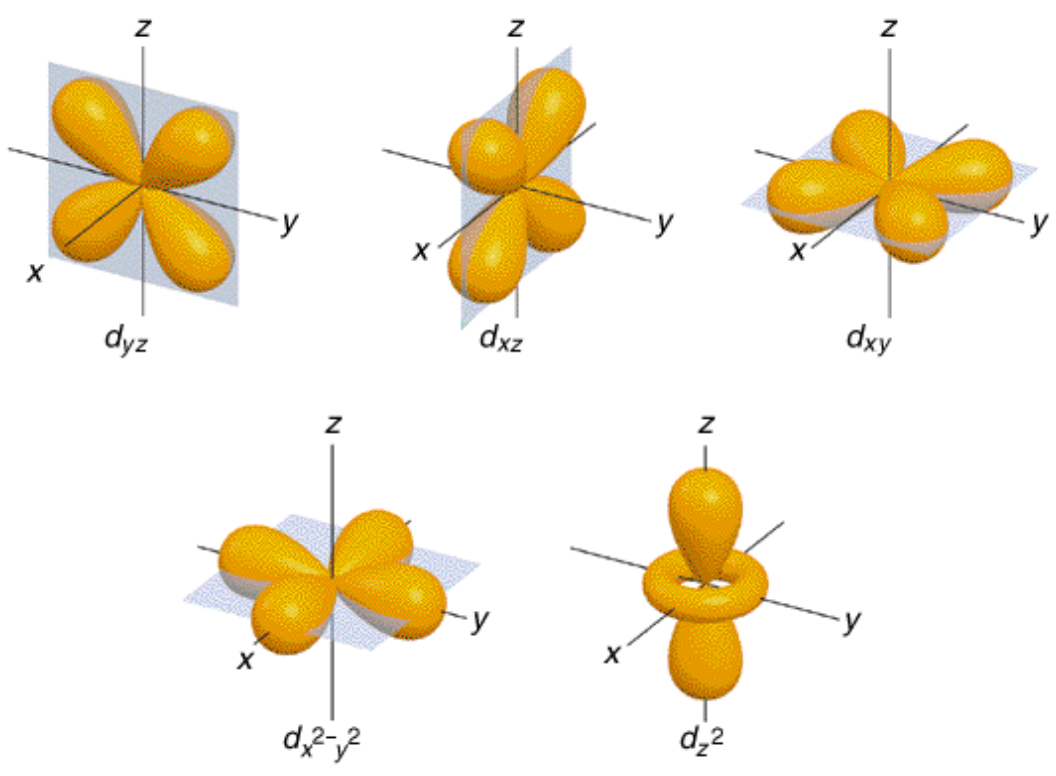

Figure 3.11. The five d-orbital wave functions, giving the spatial distribution of valence electrons. This figure was taken from Professor Scott Oliver (www.chemistry.ucsc.edu/ soliver)

The five d-orbital wave functions are given in terms of $\left|\hat{L}, \hat{L}_{z}\right\rangle$.

$$
\begin{aligned}
& d_{z^{2}}=|2,0\rangle \\
& d_{x y}=\frac{-i}{\sqrt{2}}(|2,2\rangle-|2,-2\rangle) \\
& d_{y z}=\frac{i}{\sqrt{2}}(|2,-1\rangle+|2,1\rangle) \\
& d_{x z}=\frac{1}{\sqrt{2}}(|2,-1\rangle-|2,1\rangle) \\
& d_{x^{2}-y^{2}}=\frac{1}{\sqrt{2}}(|2,2\rangle+|2,-2\rangle)
\end{aligned}
$$

These functions are each a superposition of the spherical harmonics. According to $\mathrm{Kasai}^{21}$, the $\mathrm{d}_{\mathrm{z}}{ }^{2}$ orbital lies highest in energy while the $\mathrm{d}_{\mathrm{x}-\mathrm{y}}^{2}{ }^{2}$ level is lowest. I will show that this is indeed true for this particular arrangement. Figure 3.12 shows how the levels are arranged. 


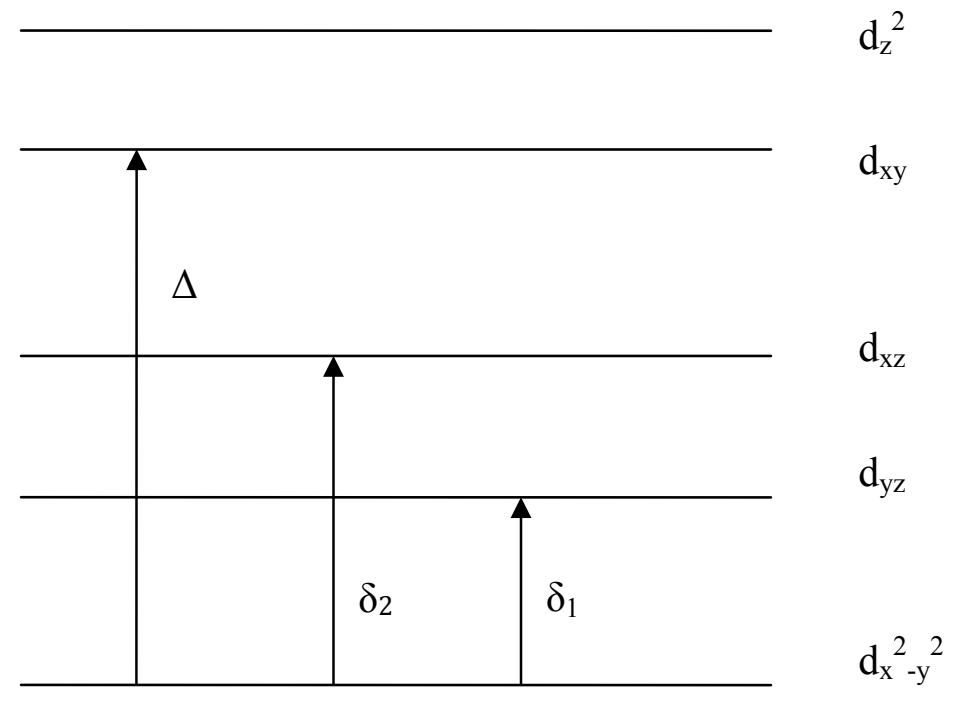

Figure 3.12. Relative energy levels of the $5 \mathrm{~d}$-orbital wave functions. $\mathrm{d}_{\mathrm{x}}{ }^{2}-\mathrm{y}$ is lowest in energy because the four oxygen ions in the [110] plane of rutile are not arranged squarely about the [110] axis.

Taking $\mathrm{d}_{\mathrm{x}-\mathrm{y}}^{2}=|G\rangle$, and using equation 3, the following expressions are derived

for $g_{x x}, g_{y y}$, and $g_{z z}$ :

$$
\begin{aligned}
& g_{x x}=g_{e}-\frac{2 \lambda}{\delta_{1}} \\
& g_{y y}=g_{e}-\frac{2 \lambda}{\delta_{2}} \\
& g_{z z}=g_{e}-\frac{8 \lambda}{\Delta}
\end{aligned}
$$

In order to account for covalency, a reduction factor $k=0.6$ was used, so that $\lambda=k \lambda$ '.

Using the known value of $155 \mathrm{~cm}^{-1}$ for $\lambda$, one gets the following results:

$$
\begin{aligned}
& \delta_{1}=3009.71 \mathrm{~cm}^{-1} \\
& \delta_{2}=6480.84 \mathrm{~cm}^{-1} \\
& \Delta=28837.2 \mathrm{~cm}^{-1}
\end{aligned}
$$

This shows that the $\mathrm{d}_{\mathrm{x}-\mathrm{y}}^{2}{ }^{2}$ level is the ground state. 


\section{Chapter 3 References}

1. P. F. Chester, J. Appl. Phys. 32, 866 (1961).

2. H. J. Gerritsen, in Paramagnetic Resonance, edited by W. Low (Academic Press, New York, 1963), Vol. 1, p. 3.

3. W. Low and E. L. Offenbacher, in Solid State Physics, edited by F. Seitz and D. Turnbull (Academic Press, New York, 1965), Vol. 17, p. 135.

4. R. R. Hasiguti, Annu. Rev. Mater. Sci. 2, 69 (1972).

5. L. N. Shen, O. W. Johnson, W. D. Ohlsen, and J. W. DeFord, Phys. Rev. B 10, $1823(1974)$.

6. J. W. DeFord and O. W. Johnson, J. Appl. Phys. 44, 3001 (1973)

7. O. W. Johnson, W. D. Ohlsen, and P. I. Kingsbury, Jr., Phys. Rev. 175, 1102 (1968).

8. S. Klauer and M. Wohlecke, Europhys. Lett. 20, 439 (1992).

9. F. Herklotz, E. V. Lavrov, and J. Weber, Phys. Rev. B 83, 235202 (2011).

10. B. H. Soffer, J. Chem. Phys. 35, 940 (1961).

11. O. W. Johnson, W. D. Ohlsen, and P. I. Kingsbury, Jr., Phys. Rev. 175, 1102 (1968).

12. J. R. Bates and R. A. Perkins, Phys. Rev. B 16, 3713 (1977).

13. Y. Chen, R. Gonzalez, and K. L. Tsang, Phys. Rev. Lett. 53, 1077 (1984).

14. S. Klauer and M. Wohlecke, Europhys. Lett. 20, 439 (1992).

15. M. Koudriachova, S. W. de Leeuw, and N. M. Harrison, Phys. Rev. B 70, 165421 (2004).

16. O. W. Johnson, J. DeFord, and J. W. Shaner, J. Appl. Phys. 44, 3008 (1973). 
17. J. A. Bolton, J. R. Wertz and J. E. Weil, Electron Paramagnetic Resonance: Elementary Theory and Practical Applications. John Wiley \& Sons, 1994.

18. V. Szalay, L. Kovács, M. Wöhlecke, and E. Libowitzky, Chem. Phys. Lett. 354, $56(2002)$.

19. Shan Yang and L. E. Halliburton, Phys. Rev. B 81, 035204 (2010).

20. Bolton, J. A., Weil, J. E. Electron Paramagnetic Resonance: Elementary Theory and Practical Applications. John Wiley \& Sons, 2007.

21. P. H. Kasai, Phys. Lett. 7, 5 (1963)

22. Shan Yang, Ph.D. Dissertation, West Virginia University (2009). 


\section{Chapter 4}

\section{Oxygen Vacancies Adjacent to Substitutional $\mathrm{Cu}^{2+}$ Ions}

\subsection{Introduction}

In the last decade, room-temperature ferromagnetism has become a topic of considerable interest due to the development of semiconductor spintronics. Spintronics explores the role of electron spin in the functionality of solid-state devices. Spintronic devices require a current of spin-polarized electrons and a device that is sensitive to the spin of an electron, i.e., a spin detector. The simplest method of producing a spinpolarized current of electrons is to pass the electrons through a ferromagnetic material. The Curie temperature required for ferromagnetic ordering in semiconductors is often in the cryogenic regime, ${ }^{1}$ which is an obvious hindrance in the development of practical semiconductor-based spintronic devices. Electron spin-polarization lifetimes are longer in semiconductors, often by several orders of magnitude when compared to metals, particularly when the electron is located near an impurity. These longer lifetimes are of particular interest in the development of quantum computers.

Several works have shown theoretically that an oxygen vacancy adjacent to a substitutional copper impurity is necessary to induce room-temperature ferromagnetism in $\mathrm{TiO}_{2}{ }^{2-6}$ As an example, Duhalde et al. ${ }^{2}$ have described the appearance of roomtemperature ferromagnetism in $\mathrm{TiO}_{2}$ thin films doped with copper. Figure 4.1, taken from the Physical Review B paper published by Duhalde et al., ${ }^{2}$ shows two hysteresis curves obtained from these samples. These data were taken at room temperature and show significant magnetization. These authors estimated a magnetic moment of $1.5 \mu_{\mathrm{B}}$ per $\mathrm{Cu}$ atom, assuming a copper concentration of 10 at. \% in a film $1000 \AA$ thick. This 
study indicates that isolated magnetic impurities are not sufficient to produce room temperature ferromagnetism in $\mathrm{TiO}_{2}$.

The two sets of data in Fig. 4.1 represent data taken from an as-grown sample (as cast) and a sample that was heated to $800{ }^{\circ} \mathrm{C}$ for 30 minutes in an oxygen-rich atmosphere (after thermal treatment). One can see that the heat-treated sample exhibits a smaller hysteresis curve than the as-cast sample. The heat-treated sample has fewer oxygen vacancies, so this hysteresis plot shows the correlation between oxygen vacancy concentration and room-temperature ferromagnetism. The magnetism is reduced significantly in the heat-treated sample.

Ab initio calculations were performed by Duhalde et al. ${ }^{2}$ to explain the magnetism exhibited by a sample with oxygen vacancies. These calculations were done using $\mathrm{Ti}_{4} \mathrm{O}_{8}$ and $\mathrm{Ti}_{3} \mathrm{CuO}_{8}$ supercells. These supercells are composed of two $\mathrm{TiO}_{2}$ unit cells stacked along the $\mathrm{c}$ axis, forming the $\mathrm{Ti}_{4} \mathrm{O}_{8}$ structure. Their calculations show that the energy

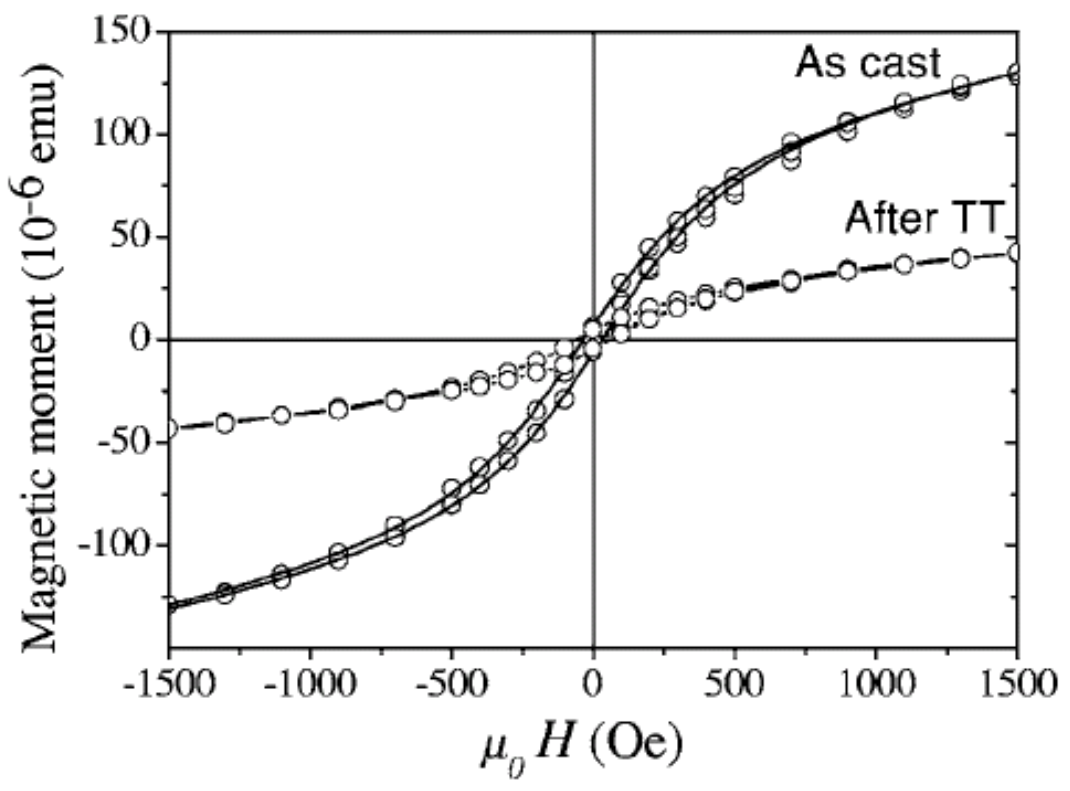

Figure 4.1. Hysteresis curves of the as-cast and heat-treated $\mathrm{TiO}_{2}: \mathrm{Cu}$ thin films. This plot was taken from Ref. 2. 
required to form an oxygen vacancy in $\mathrm{Cu}$-doped $\mathrm{TiO}_{2}$ is $4 \mathrm{eV}$, lower than the required $10 \mathrm{eV}$ for an undoped sample. The energy required to replace a Ti atom with a $\mathrm{Cu}$ atom is also lower when there is an oxygen vacancy present. Their calculations show that no magnetism is found when the sample contains no oxygen vacancies. This is in contrast to the case where oxygen vacancies are present. In that case, a magnetic moment of $1.0 \mu_{\mathrm{B}}$ per supercell is reported, nearly independent of the location of the oxygen vacancy around the $\mathrm{Cu}$ ion.

Previous investigators ${ }^{2-6}$ have shown that a substitutional copper impurity next to an oxygen vacancy is energetically favorable, and necessary to achieve room-temperature ferromagnetism in $\mathrm{Cu}$-doped $\mathrm{TiO}_{2}$. However, there is little experimental evidence to support this model. ${ }^{7}$ The present chapter is an EPR and ENDOR study of substitutional copper impurities $\left(\mathrm{Cu}^{2+}, 3 \mathrm{~d}^{9}\right)$ in $\mathrm{TiO}_{2}$ rutile. Principal values and principal axis directions for the g, hyperfine, and nuclear quadrupole matrices for both copper isotopes are determined. Also, the important question of the environment surrounding the $\mathrm{Cu}^{2+}$ impurity is addressed through a photoexcitiation experiment involving the $\mathrm{Cu}^{2+}, \mathrm{Fe}^{3+}$, and $\mathrm{Cr}^{3+}$ EPR signals, as well as previously studied EPR signals due to singly and doubly ionized oxygen vacancies. The behavior of these EPR signals when monitored simultaneously during $442 \mathrm{~nm}$ laser light illumination provides evidence that the $\mathrm{Cu}^{2+}$ ion is located next to an oxygen vacancy.

\subsection{Samples}

The rutile samples used in this study were obtained from two commercial crystal growth companies, CrysTec in Germany and Nakazumi in Japan. These crystals were 
grown using the Verneuil method, and they both contained copper impurities that were unintentionally introduced during the growth process. They also contained $\mathrm{Fe}^{3+}$ and $\mathrm{Cr}^{3+}$ impurities. The concentration of each of these impurities is on the order of $1 \mathrm{ppm}$.

\subsection{EPR Results}

Figure 4.2 is an EPR spectrum of $\mathrm{Cu}^{2+}$ in $\mathrm{TiO}_{2}$ taken at $18 \mathrm{~K}$, which is the optimum temperature for observing this signal. Above $40 \mathrm{~K}$, the signal becomes broad and difficult to detect, although the $\mathrm{Cu}^{2+}$ ions are still present. Below approximately 12 $\mathrm{K}$, the signal is easily saturated with microwave power. The $\mathrm{Cu}^{2+}$ ions sit on $\mathrm{Ti}^{4+}$ sites in

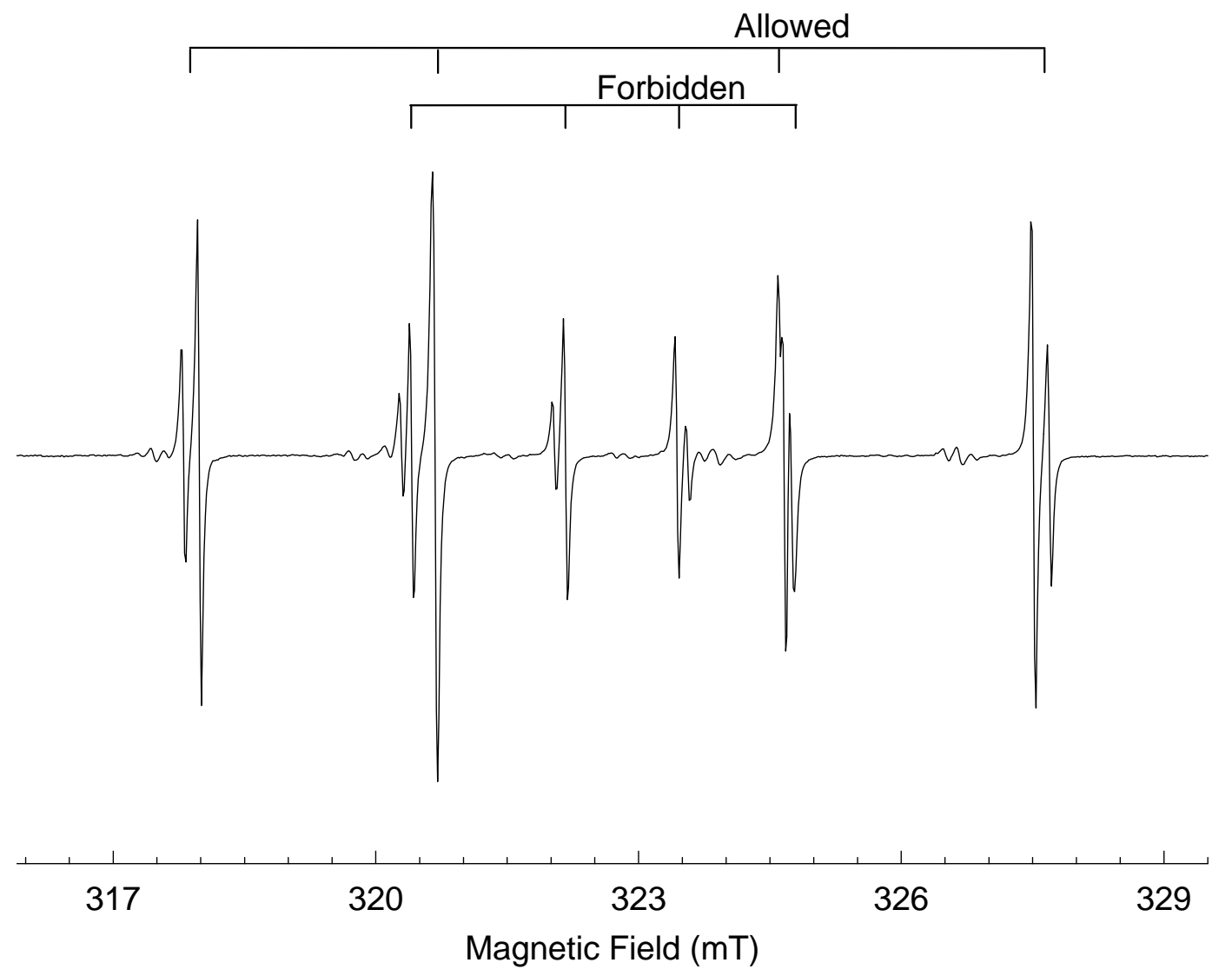

Figure 4.2. EPR spectrum of $\mathrm{Cu}^{2+}$ in $\mathrm{TiO}_{2}$ with the magnetic field parallel to the [001] axis. Stick diagrams indicate "allowed" and "forbidden" transitions. This spectrum was taken at $18 \mathrm{~K}$, and the microwave frequency was $9.4717 \mathrm{GHz}$. 
the rutile lattice, next to a doubly ionized oxygen vacancy (i.e., a vacancy with no trapped electrons). This $\left(\mathrm{Cu}^{2+}-\mathrm{V}_{\mathrm{O}}\right)$ complex has the same electric charge as the $\mathrm{Ti}^{4+}$ and $\mathrm{O}^{2-}$ ions initially present. The doubly ionized oxygen vacancy has an effective $2+$ charge.

The $\left(\mathrm{Cu}^{2+}-\mathrm{V}_{\mathrm{O}}\right)$ complex is paramagnetic. This $3 \mathrm{~d}^{9}$ configuration has one unpaired electron $(\mathrm{S}=1 / 2)$. Figure 4.2 shows a set of four lines for each isotope of copper. Both isotopes of copper have a nuclear spin $I=3 / 2$. Thus, the spectrum in Fig. 4.2 has four "allowed" lines for each isotope occurring at $\mathrm{g}\left({ }^{63} \mathrm{Cu}\right)=2.09280$ and $\mathrm{g}\left({ }^{65} \mathrm{Cu}\right)=2.09281$ for the magnetic field along the [001] direction. Additional lines appearing in Fig. 4.2 are due to "forbidden" transitions, i.e., transitions that do not obey the usual EPR selection rules $\left(\Delta \mathrm{m}_{\mathrm{S}}= \pm 1, \Delta \mathrm{m}_{\mathrm{I}}=0\right)$. Stick diagrams above the data in Fig. 4.2 indicate the approximate region where the ${ }^{63} \mathrm{Cu}$ and ${ }^{65} \mathrm{Cu}$ allowed transitions and forbidden transitions appear. ${ }^{65} \mathrm{Cu}$ has a slightly larger magnetic moment than ${ }^{63} \mathrm{Cu}$, and this results in a larger splitting between hyperfine lines. The natural abundance of ${ }^{65} \mathrm{Cu}$ is $30.8 \%$, compared to $69.2 \%$ for ${ }^{63} \mathrm{Cu}$. Therefore, the smaller, outermost lines in Fig. 4.2 are assigned to ${ }^{65} \mathrm{Cu}$ nuclei, and the next two larger lines are due to ${ }^{63} \mathrm{Cu}$. The inner pair of ${ }^{65} \mathrm{Cu}$ lines are somewhat obstructed by forbidden transitions. These forbidden transitions are $\Delta \mathrm{m}_{\mathrm{S}}= \pm 1$, $\Delta \mathrm{m}_{\mathrm{I}}= \pm 2$ transitions.

Figure 4.3 shows the $\mathrm{Cu}^{2+}$ spectrum when the magnetic field is aligned along the [100] axis. The forbidden transitions indicated by the stick diagrams above this spectrum are $\Delta \mathrm{m}_{\mathrm{S}}= \pm 1, \Delta \mathrm{m}_{\mathrm{I}}= \pm 1$ transitions. The spectrum was taken at $18 \mathrm{~K}$ and at a microwave frequency of $9.4749 \mathrm{GHz}$. In this spectrum, one can easily see the two sets of four EPR lines corresponding to the two isotopes of copper. A stick diagram above the data in Fig. 4.3 indicates the allowed transtitions. One set of lines for each isotope appears because 


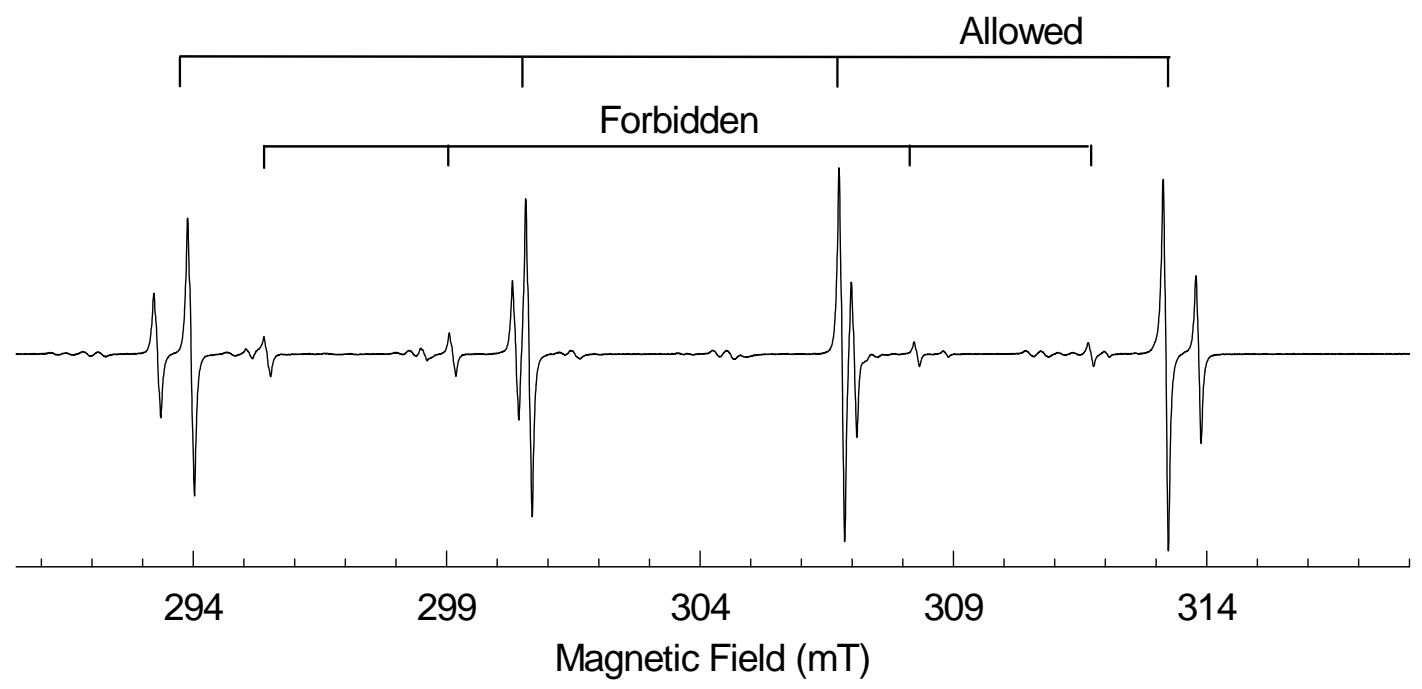

Figure 4.3. EPR spectrum of $\mathrm{Cu}^{2+}$ in $\mathrm{TiO}_{2}$ with the magnetic field parallel to the [100] axis. This spectrum was taken at $18 \mathrm{~K}$ and at a microwave frequency of $9.4749 \mathrm{GHz}$.

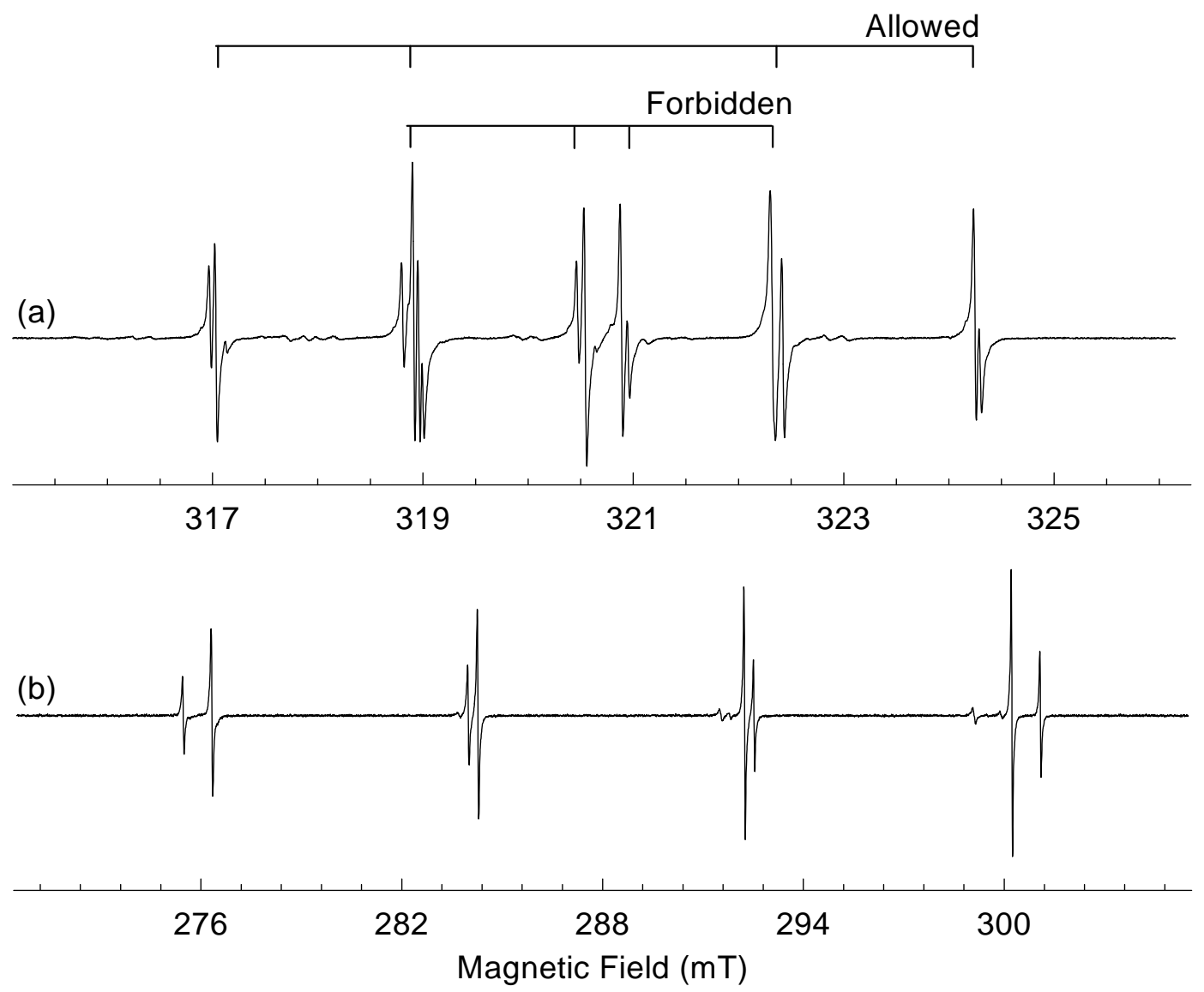

Figure 4.4. EPR spectrum of $\mathrm{Cu}^{2+}$ in $\mathrm{TiO}_{2}$ with the magnetic field parallel to the [110] axis. Two sets of lines appear, as the two copper sites are not magnetically equivalent for this field orientation. The lines in trace (b) are all "allowed" lines. 
the magnetic field is oriented such that both $\mathrm{Ti}^{4+}$ sites are magnetically equivalent.

Figure 4.4 was taken with the magnetic field aligned along the [110] crystal axis, and looks strikingly different from the c-axis and [100] spectra. The spectrum contains two sets of $\mathrm{Cu}$ lines, and is divided into upper and lower segments for easier viewing in Fig 4.4. The appearance of two sets of lines, at higher and lower field, indicates that there are two magnetically inequivalent sites (orientations) of the $\mathrm{Cu}^{2+}$ defect when the field is aligned along the [110] direction. Forbidden transitions in Fig. 4.4(a) are $\Delta \mathrm{m}_{\mathrm{S}}=$ $\pm 1, \Delta \mathrm{m}_{\mathrm{I}}= \pm 1$ transitions. Forbidden transitions in Fig. 4.4(b) have zero intensity and are not seen.

\subsection{Spin Hamiltonian Analysis}

Ensign et al. ${ }^{8}$ calculated g, hyperfine, and nuclear quadrupole parameters for $\mathrm{Cu}^{2+}$ in rutile. However, So and Belford ${ }^{9}$ commented that the nuclear quadrupole parameters were not accurately calculated using second order perturbation theory. Using exact matrix diagonalization, we obtained principal values for the $\mathrm{g}, \mathrm{A}$, and $\mathrm{P}$ matrices. The principal axes of these three matrices are collinear and coincide with the high-symmetry directions of the crystal. Therefore, Euler angles are not necessary to specify the orientation of the principal axes.

The following spin Hamiltonian was used to analyze the EPR and ENDOR spectra.

$$
\hat{H}=\mu_{\mathrm{B}} \vec{B} \cdot \vec{g} \cdot \vec{S}+\vec{S} \cdot \vec{A} \cdot \vec{I}+\vec{I} \cdot \vec{Q} \cdot \vec{I}-g_{n} \mu_{n} \vec{B} \cdot \vec{I}
$$

Here, the electron Zeeman, hyperfine, nuclear quadrupole, and nuclear Zeeman terms are included. Sixteen parameters are needed to fully characterize the $\mathrm{Cu}$-related defect. 
Three principal g values, three principal A values, and two principal $Q$ values are needed to describe each copper isotope. The Q matrix is traceless, so the third principal value of this matrix can be obtained from the other two. The EPR angular dependence for two crystal planes is shown in Fig. 4.5. Figure 4.5 only shows the angular dependence of the allowed ${ }^{63} \mathrm{Cu}$ transitions for ease of viewing. The solid lines were obtained using the "best" values for $\mathrm{g}, \mathrm{A}$, and $\mathrm{Q}$. These values were obtained using a least squares fitting routine and are shown in Table 4.1. The coordinate system chosen is right-handed and has $\mathrm{z}$ along the [110] direction and y along the [001].

The fitting procedure was done using only the angular dependence of the EPR signal. This is acceptable here because the EPR lines are well resolved for both isotopes and forbidden transitions were easily identified. The four allowed and four forbidden lines in Fig. 4.2, four allowed and four forbidden lines in Fig. 4.3, four allowed and four forbidden lines in Fig. 4.4(a) and four allowed lines in Fig. 4.4(b) were all used to

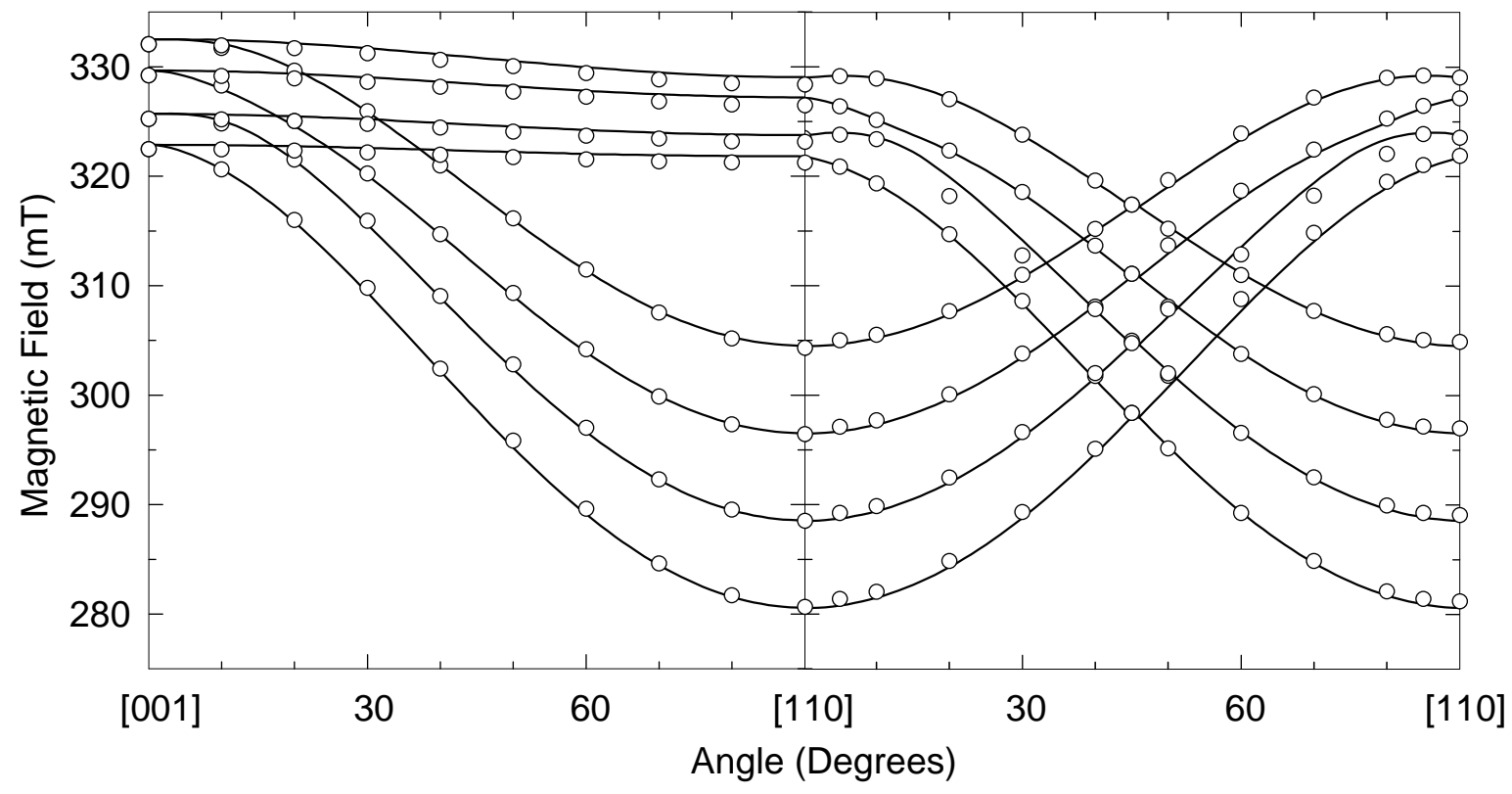

Figure 4.5. EPR angular dependence of $\mathrm{Cu}^{2+}$ in $\mathrm{TiO}_{2}$. Circles indicate experimental data points while solid lines are computer-generated "best fit" lines. 
Table 4.1. Spin-Hamiltonian parameters for the two isotopes of $\mathrm{Cu}^{2+}$ in $\mathrm{TiO}_{2}$ (rutile). Error limits are \pm 0.00001 for the $\mathrm{g}$ values, $\pm 0.3 \mathrm{MHz}$ for the A values and $\pm 0.2 \mathrm{MHz}$ for the $\mathrm{P}$ values.

\begin{tabular}{|c|c|c|c|}
\hline g matrix & ${ }^{63} \mathrm{Cu}$ & ${ }^{65} \mathrm{Cu}$ & Principal-axis direction \\
\hline$g_{x}$ & 2.10699 & 2.10697 & {$[\overline{1} 10]$} \\
\hline $\mathrm{g}_{\mathrm{y}}$ & 2.09281 & 2.09280 & {$[001]$} \\
\hline $\mathrm{g}_{\mathrm{z}}$ & 2.34518 & 2.34516 & [110] \\
\hline \multicolumn{4}{|l|}{ A matrix } \\
\hline $\mathrm{A}_{\mathrm{x}}$ & $55.35 \mathrm{MHz}$ & $59.2 \mathrm{MHz}$ & {$[\overline{1} 10]$} \\
\hline $\mathrm{A}_{\mathrm{y}}$ & $82.34 \mathrm{MHz}$ & $88.21 \mathrm{MHz}$ & [001] \\
\hline $\mathrm{A}_{\mathrm{z}}$ & $-261.98 \mathrm{MHz}$ & $-280.83 \mathrm{MHz}$ & {$[110]$} \\
\hline \multicolumn{4}{|l|}{$\mathrm{P}$ matrix } \\
\hline $\mathrm{P}_{\mathrm{x}}$ & $-10.95 \mathrm{MHz}$ & $-10.11 \mathrm{MHz}$ & {$[\overline{1} 10]$} \\
\hline $\mathrm{P}_{\mathrm{y}}$ & $-9.23 \mathrm{MHz}$ & $-8.51 \mathrm{MHz}$ & {$[001]$} \\
\hline $\mathrm{P}_{\mathrm{z}}$ & $20.18 \mathrm{MHz}$ & $18.62 \mathrm{MHz}$ & [110] \\
\hline
\end{tabular}

perform the fitting. This gave 28 lines for each isotope, and the fitting for each isotope was performed independently.

In this particular study, relative signs of the hyperfine and quadrupole parameters can be determined from the EPR spectra, but absolute signs cannot. More generally, EPR measurements of the relative intensity of individual lines at very low temperature (less 
than $4 \mathrm{~K}$ ) can sometimes provide absolute signs. In an early study of the relative signs of $\mathrm{Cu}^{2+}$ ions in another material, Bleaney et al. ${ }^{10-12}$ found that $\mathrm{P}_{\mathrm{x}}$ and $\mathrm{A}_{\mathrm{x}}$ have opposite signs, $\mathrm{P}_{\mathrm{y}}$ and $\mathrm{A}_{\mathrm{y}}$ have opposite signs, and $\mathrm{P}_{\mathrm{z}}$ and $\mathrm{A}_{\mathrm{z}}$ have opposite signs. Because the $\mathrm{P}$ matrix is traceless in its principal axis frame, one can conclude that $\mathrm{P}_{\mathrm{x}}$ and $\mathrm{P}_{\mathrm{y}}$ are opposite in sign to $\mathrm{P}_{\mathrm{z}}$. These determinations were made by Bleaney et al. after observing the forbidden transitions when the magnetic field was slightly rotated from high symmetry directions of their crystals. From their work, a negative sign is assigned to $A_{z}$ while $A_{x}$ and $\mathrm{A}_{\mathrm{y}}$ are given positive signs.

The ratios $\left({ }^{65} \mathrm{~A}_{\mathrm{i}} /{ }^{63} \mathrm{~A}_{\mathrm{i}}\right)$ for $\mathrm{i}=\mathrm{x}, \mathrm{y}$ and $\mathrm{z}$ are $1.070,1.071$, and 1.072 , respectively. These ratios are in good agreement with the ratio of the nuclear $g$ factors of the two copper isotopes $(1.588 / 1.484=1.070)$. The ratios of the principal quadrupole values $\left({ }^{65} \mathrm{P}_{\mathrm{i}} /{ }^{63} \mathrm{P}_{\mathrm{i}}\right)$ are $0.923,0.922$, and 0.923 , which agrees closely to the ratio of nuclear electric quadrupole moments of the two isotopes $\left(-0.204 * 10^{-28} /-0.220 * 10^{-28}=0.927\right)$. The agreement found in these ratios provides a check of the accuracy of the principal values listed in Table 4.1.

\subsection{ENDOR Results}

ENDOR was performed on the $\mathrm{Cu}^{2+}$ EPR signals in $\mathrm{TiO}_{2}$. While ENDOR data were not needed to perform the least squares fitting procedure that determined the $\mathrm{g}, \mathrm{A}$, and $\mathrm{P}$ parameters, they do provide an experimental check of the values. Figure 4.6 shows two ENDOR spectra taken at $15 \mathrm{~K}$ and with the magnetic field oriented along the [001] crystal direction. Trace (a) was taken with the magnetic field fixed on the lowest-field

${ }^{63} \mathrm{Cu}$ EPR line in Fig. 4.2 and trace (b) was taken with the field fixed on the highest-field 
${ }^{63} \mathrm{Cu}$ EPR lines in Fig. 4.2.

A pair of ENDOR lines appears for each EPR line. Using the parameters from Table 4.1, one can check that the lines appear at the correct position. In trace (a), the ENDOR lines appear at 14.54 and 66.23 MHz. Using the parameters in Table 1, exact diagonalization predicts that the ENDOR lines will appear at $14.70 \mathrm{MHz}$ and $66.54 \mathrm{MHz}$. In trace (b), the ENDOR lines appear at 22.70 MHz and 61.35 MHz. Exact diagonalization predicts that they should appear at 22.23 MHz and 61.12 MHz. The difference between the predicted and experimentally determined values in each trace is less than the linewidths, which are approximately $700-800 \mathrm{kHz}$.

Figure 4.7 shows an ENDOR spectra taken with the magnetic field aligned along the [100] axis. The two traces, (a) and (b), were taken while sitting on the lowest- and

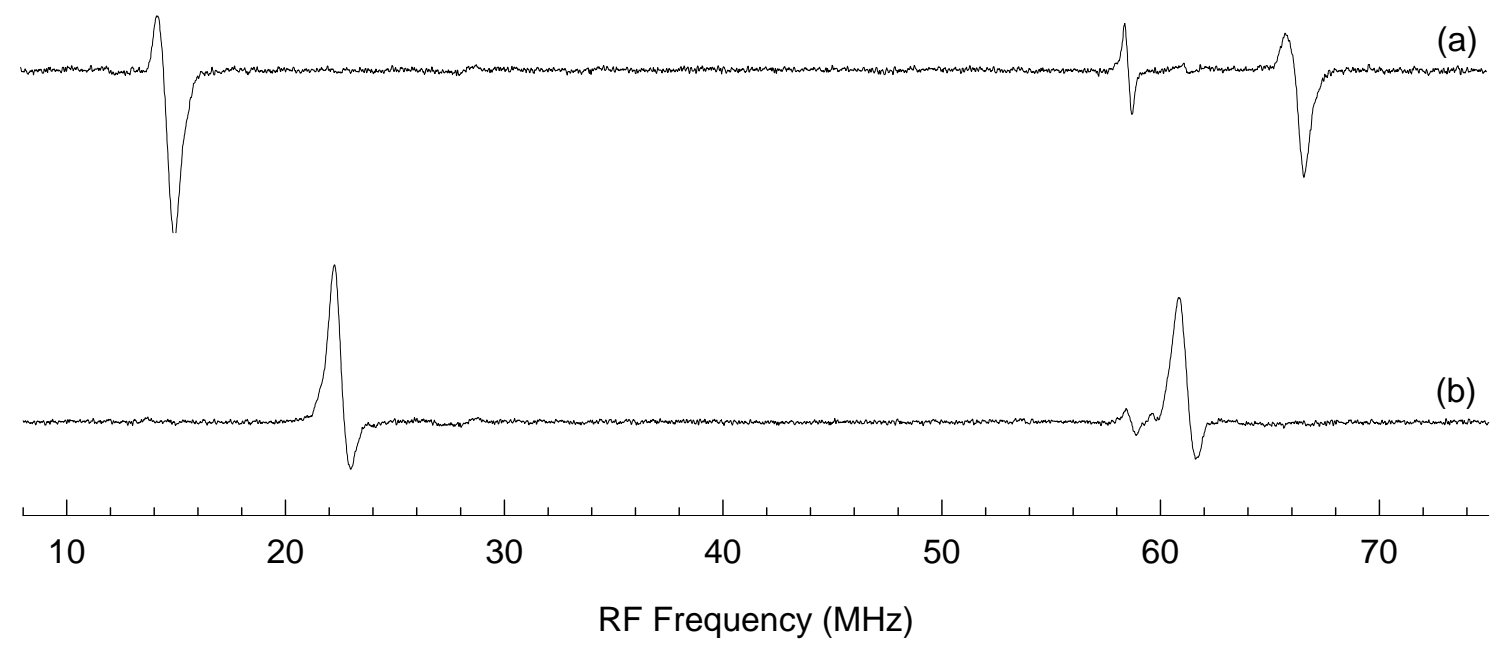

Figure 4.6. ${ }^{63} \mathrm{Cu}$ ENDOR spectra taken with the magnetic field oriented along the [001] crystal direction. Trace (a) was taken with the field fixed at $316.146 \mathrm{mT}$, which corresponds to the lowest-field allowed line in Figure 4.2. Trace $\mathrm{b}$ was taken with the field fixed at $325.707 \mathrm{mT}$, corresponding to the highest-field allowed line in Fig. 4.2. 


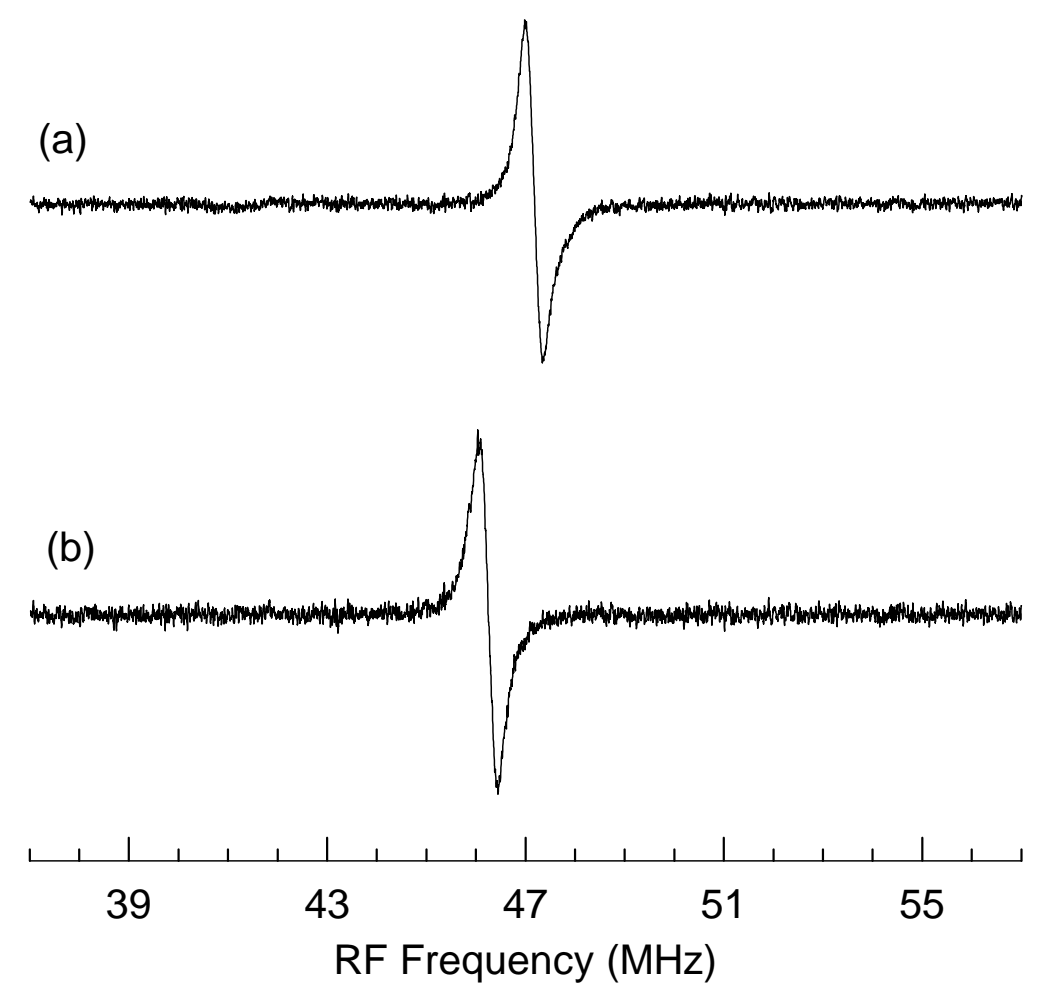

Figure 4.7. ${ }^{63} \mathrm{Cu}$ ENDOR spectra taken with the magnetic field oriented along the [100] crystal direction. Trace (a) was taken with the field fixed at $292.455 \mathrm{mT}$, which corresponds to the lowest-field allowed line in Figure 4.3. Trace $b$ was taken with the field fixed at $311.775 \mathrm{mT}$, corresponding to the highest allowed line in Figure 4.3.

highest-field ${ }^{63} \mathrm{Cu}$ EPR lines in Fig. 4.3, respectively. Only one line appears in the two traces because the upper radio frequency limit of the spectrometer prevented observation of the second line. The line in Fig. 4.7(a) appears at 47.17 MHz. Exact diagonalization predicts that the line should appear at $46.83 \mathrm{MHz}$. The observed position of the line in Fig. 4.7(b) is $46.25 \mathrm{MHz}$, while the predicted position is $45.88 \mathrm{MHz}$. The differences in the predicted and observed positions are again within the linewidths.

\subsection{Photoinduced Changes in Charge States}

When the $\mathrm{TiO}_{2}$ crystal is illuminated with $442 \mathrm{~nm}$ laser light, the $\mathrm{Cu}^{2+} \mathrm{EPR}$ signal 
decreases in intensity by more than $50 \%$. This indicates that the laser light converts the defect into a nonparamagnetic charge state. The singly and doubly ionized oxygen vacancy signals that were first reported by Yang et al. ${ }^{13}$ also appear. Together, these observations suggest that there is a correlation between the charge states of the $\mathrm{Cu}^{2+}$ defect and oxygen vacancies in $\mathrm{TiO}_{2}$. Figure 4.8 contains three traces that show the
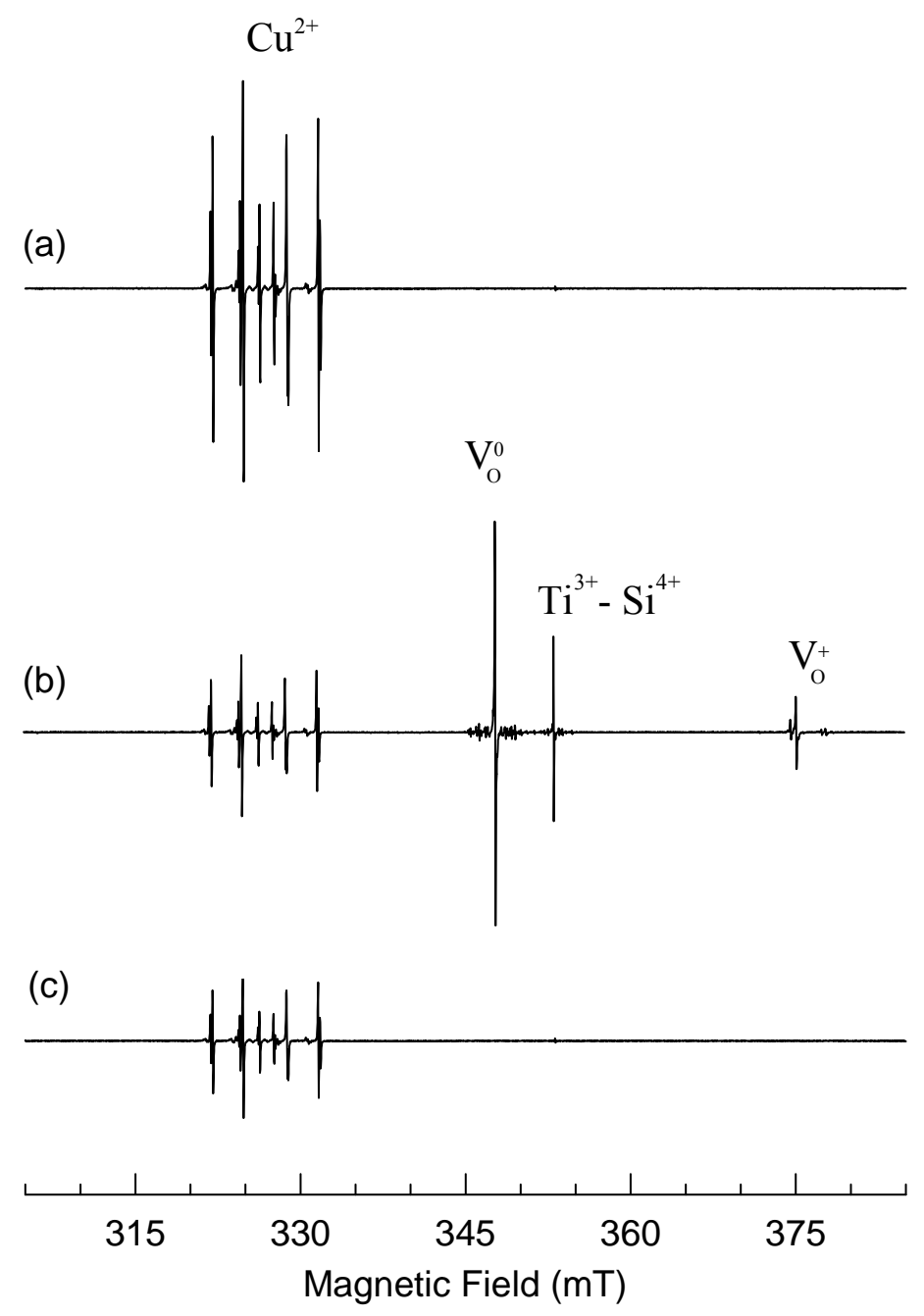

Figure 4.8. The effect of laser illumination on $\mathrm{Cu}^{2+}$. Trace a was taken prior to illumination. Trace $\mathrm{b}$ was taken immediately after the laser was turned off. Trace (c) was taken after the crystal was warmed to $60 \mathrm{~K}$ for 1 minute and without illumination. All three traces were taken at $20 \mathrm{~K}$ and with the same spectrometer parameters, and with the magnetic field aligned along the [001] axis. 
effects of laser illumination. Trace (a) was taken prior to illumination, trace (b) was taken during $442 \mathrm{~nm}$ laser illumination, and trace (c) was taken following the illumination and after the crystal was warmed to $60 \mathrm{~K}$ for 1 minute. Figure 4.8 illustrates the reduction of the $\mathrm{Cu}^{2+}$ signal when the laser is turned on. The three signals that appear in trace $(b)$ are the doubly ionized oxygen vacancy $\left(\mathrm{V}_{\mathrm{O}}{ }^{0}\right)$, the silicon-related $\mathrm{Ti}^{3+}$ center $\left(\mathrm{Ti}^{3+}-\mathrm{Si}^{4+}\right)$, and the singly ionized oxygen vacancy $\left(\mathrm{V}_{\mathrm{O}}{ }^{+}\right)$. Only one of the two lines of the doubly ionized $(S=1) V_{O}$ is shown for clarity.

Laser light illumination also results in decreased intensity of EPR signals related to $\mathrm{Fe}^{3+}$ and $\mathrm{Cr}^{3+}$ defects. Figure 4.9 shows three traces of these two signals under the same conditions as the traces in Fig. 4.8. $\mathrm{Fe}^{3+}$ and $\mathrm{Cr}^{3+}$ are deep acceptors (hole traps) in rutile. The reduction in intensity when the laser is turned on suggest that some of the $\mathrm{Fe}^{3+}$ and $\mathrm{Cr}^{3+}$ centers release an electron and convert to $\mathrm{Fe}^{4+}$ and $\mathrm{Cr}^{4+}$. Warming the crystal to $60 \mathrm{~K}$ results in a partial recovery of these signals. This recovery step coincides with the disappearance of the oxygen vacancy and $\mathrm{Ti}^{3+}-\mathrm{Si}^{4+}$ signals. This suggests that the electrons trapped near the oxygen vacancies and silicon centers are released and are re-trapped by the iron and chromium, converting them back to the $3+$ charge state.

Figure 4.8 shows that copper can exist in more than one charge state in $\mathrm{TiO}_{2}$ rutile. Laser light converts copper from its paramagnetic $2+$ charge state into a nonparamagnetic charge state, either $\mathrm{Cu}^{3+}$ or $\mathrm{Cu}^{+}$. This raises the question of whether $\mathrm{Cu}^{2+}$ is a hole trap or an electron trap in $\mathrm{TiO}_{2}$. This question is answered in Fig. 4.8(c). One can see that there is very little change in the intensity of the $\mathrm{Cu}^{2+}$ signal after the sample is warmed to $60 \mathrm{~K}$. If copper existed in the $3+$ charge state after illumination, some of the electrons from the oxygen vacancies and silicon center would have returned to the 

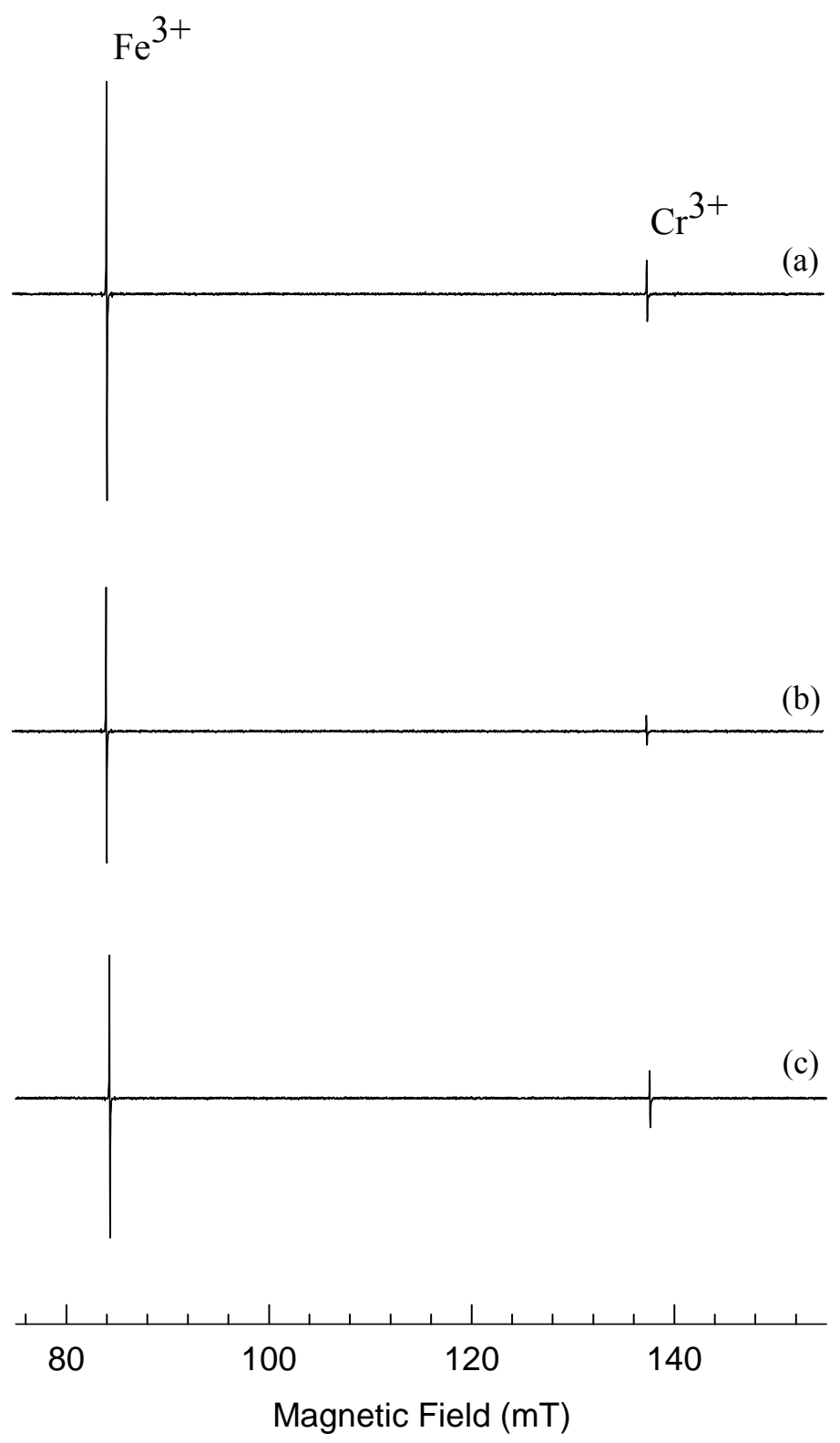

Figure 4.9. The effect of laser illumination on $\mathrm{Fe}^{3+}$ and $\mathrm{Cr}^{3+}$ in $\mathrm{TiO}_{2}$. Each trace was taken along with the corresponding trace in Fig. 4.8. The two signals here both decrease by about $50 \%$ when the laser is turned on and only partially recover after warming to $60 \mathrm{~K}$. All three traces are on the same vertical scale.

copper, resulting in the creation of more $\mathrm{Cu}^{2+}$ centers thus increasing the intensity of the $\mathrm{Cu}^{2+}$ EPR signal. The fact that this is not observed strongly suggests that the copper is converted to $\mathrm{Cu}^{+}$upon illumination, and is therefore unchanged by the release of electrons from the oxygen vacancy and silicon centers. A further pulsed anneal study 
was carried out and it showed that the $\mathrm{Fe}^{3+}, \mathrm{Cr}^{3+}$, and $\mathrm{Cu}^{2+}$ signals all return to their preillumination state at the same point above $100 \mathrm{~K}$. This behavior indicates that the electrons from the $\mathrm{Cu}^{+}$center are released and recombine with the remaining $\mathrm{Fe}^{4+}$ and $\mathrm{Cr}^{4+}$, resulting in full recovery of all three EPR signals. This study establishes that $\mathrm{Cu}^{2+}$ is an electron trap in $\mathrm{TiO}_{2}$ rutile.

\subsection{Reduction Effects}

Additional evidence that $\mathrm{Cu}^{2+}$ is an electron trap is seen when a reduction treatment is performed on $\mathrm{TiO}_{2}$. Reduction in flowing nitrogen gas at $600 \mathrm{~K}$ results in the $\mathrm{Cu}^{2+}$ EPR signal disappearing completely. The reduction treatment produces oxygen vacancies which increases the number of shallow donor states near the conduction band (i.e., raises the Fermi level). $\mathrm{Cu}^{2+}$ defects in the reduced crystal trap these "extra" free electrons, converting to $\mathrm{Cu}^{+}$. When the crystal is then oxidized at $700 \mathrm{~K}$, the $\mathrm{Cu}^{2+} \mathrm{EPR}$ signal reappears at the pre-reduction intensity. Oxidation allows oxygen ions to diffuse back into the crystal and fill the oxygen vacancies. This removes the shallow donor states (lowers the Fermi level) and allows the $\mathrm{Cu}^{+}$ions to convert back to $\mathrm{Cu}^{2+}$ ions.

\subsection{Discussion and Conclusions}

The principal axis directions of the $\mathrm{Cu}^{2+}$ hyperfine and nuclear quadrupole matrices coincide with the high-symmetry directions of the crystal, establishing that $\mathrm{Cu}^{2+}$ ions substitute for $\mathrm{Ti}^{4+}$ ions in rutile. Laser illumination and reduction experiments show that $\mathrm{Cu}^{2+}$ is an electron trap, converting to $\mathrm{Cu}^{+}$when trapping "free" electrons. These observations allow one to conclude that the $\mathrm{Cu}^{2+}$ defect is adjacent to an oxygen vacancy. 
It is unlikely that $\mathrm{Cu}^{+}$would exist in isolation, as (in an ionic picture) this would deviate by three units of charge from the host cation, $\mathrm{Ti}^{4+}$, resulting in a large energy of formation. Instead, the data suggests an electrically neutral $\mathrm{Cu}^{2+}-\mathrm{V}_{\mathrm{O}}$ complex forms during crystal growth. Laser illumination and reduction result in the formation of nonparamagnetic $\mathrm{Cu}^{+}-\mathrm{V}_{\mathrm{O}}$ complexes. The oxygen vacancy must be at one of the two oxygen ion sites along the elongation direction of the $\mathrm{TiO}_{6}$ octahedron in order to be consistent with the assignment of the $g$ and hyperfine principal axis directions. Figure 4.10 is a model of the unit cell with a visual representation of the $\mathrm{Cu}^{2+}-\mathrm{V}_{\mathrm{O}}$ defect model. Further evidence for a $\mathrm{Cu}^{2+}-\mathrm{V}_{\mathrm{O}}$ model could come by studying the electric field gradient. Our reliable principal values for the P matrix (in Table 4.1) could be compared

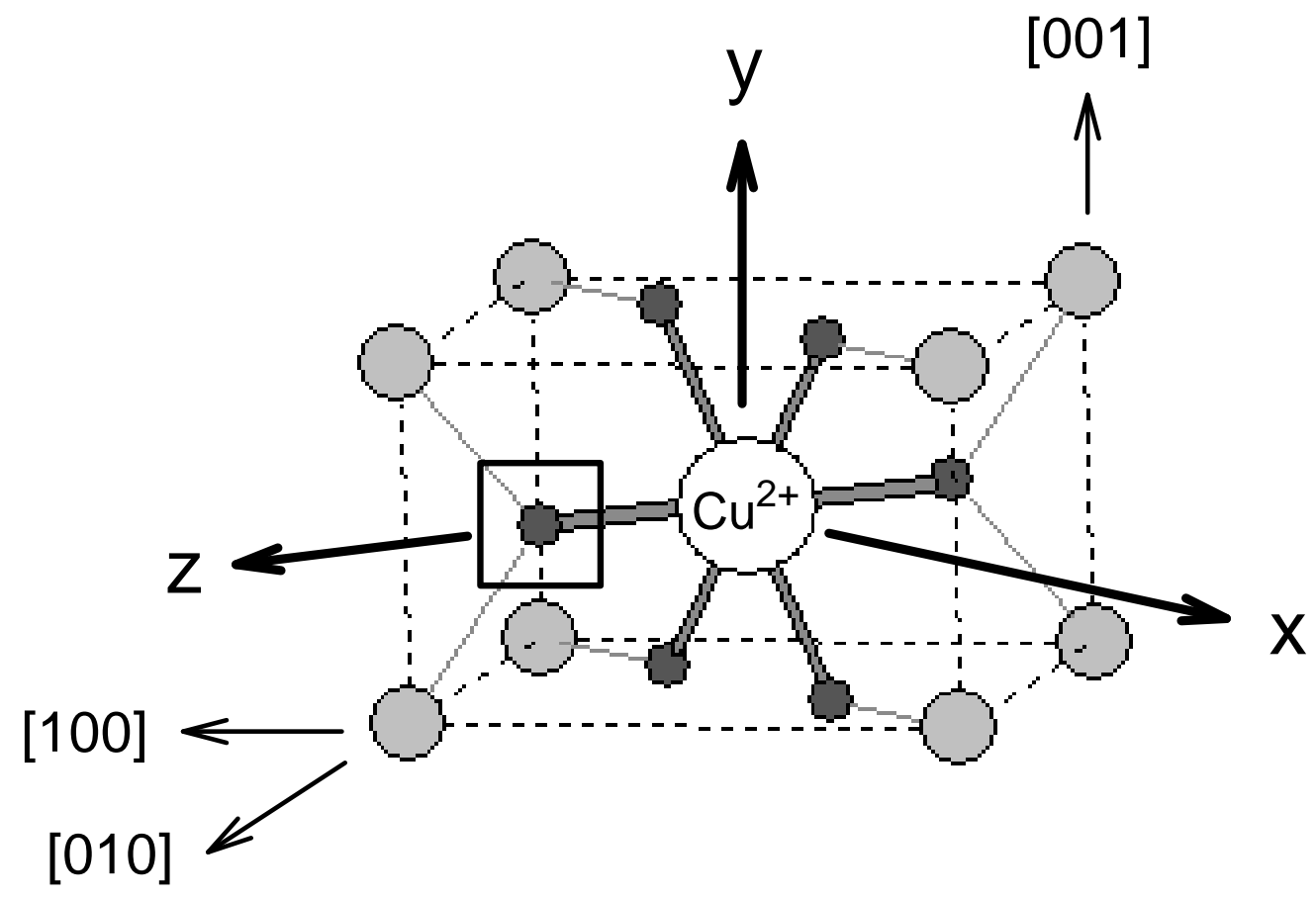

Figure 4.10. The $\mathrm{Cu}^{2+}-\mathrm{V}_{\mathrm{O}}$ defect model, with the principal axes of the $\mathrm{g}, \mathrm{A}$, and $\mathrm{P}$ matrices. The black square indicates the location of the oxygen vacancy. 
to results from first-principles calculations for the $\mathrm{Cu}$ nucleus. These calculations could be carried out with and without the oxygen vacancy and compared to our experimentally determined P values. Such calculations would provide additional evidence for or against this model of a $\mathrm{Cu}^{2+}$ ion next to an oxygen vacancy. 


\section{Chapter 4 References}

1. J. D. Bryan, S. M. Heald, S. A. Chambers, and D. R. Gamelin, J. Am. Chem. Soc. 126, 11640 (2004).

2. S. Duhalde, M. F. Vignolo, F. Golmar, C. Chiliotte, C. E. Rodriguez Torres, L. A. Errico, A. F. Cabrera, M. Renteria, F. H. Sanchez, and M. Weissmann, Phys. Rev. B. $72(2005) 161313$.

3. L. A. Errico, M. Renteria, and M. Weissmann, Phys. Rev. B 72, 184425 (2005).

4. Deng Lu Hou, Rui Bin Zhao, Hai Juan Meng, Li Yun Jia, Xiao Juan Ye, Hong Juan Zhou, and Xiu Ling Li, Thin Solid Films 516, 3223 (2008).

5. Q. K. Li, B. Wang, C. H. Woo, H. Wang, Z. Y. Zhu, and R. Wang, Europhys. Lett. 81, 17004 (2008).

6. Dongyoo Kim, Jisang Hong, Young Ran Park, and Kwang Joo Kim, J. Phys.: Condens. Matter 21, 195405 (2009).

7. M. You, T. G. Kim, and Y. M. Sung, Cryst. Growth Des. 10, 983 (2010).

8. T. C. Ensign, Te-Tse Chang, and A. H. Kahn, Phys. Rev. 188, 703 (1969).

9. H. So and R. Linn Belford, Phys. Rev. B 2, 3810 (1970).

10. B. Bleaney, K. D. Bowers, and D. J. E. Ingram, Proc. R. Soc. London A228, 147 (1955).

11. B. Bleaney, K. D. Bowers, and R. S. Trenam, Proc. R. Soc. London A228, $157(1955)$.

12. B. Bleaney, K. D. Bowers, and M. H. L. Pryce, Proc. R. Soc. London A228, 166 (1955). 
13. S. Yang, L. E. Halliburton, A. Manivannan, P. H. Bunton, D. B. Baker, M.

Klemm, S. Horn, and A. Fujishima, Appl. Phys. Lett. 94, 162114 (2009). 


\section{Chapter 5}

\section{Characterization of Interstitial Lithium Ions Adjacent to $\mathrm{Ti}^{3+}$ ions in $\mathrm{TiO}_{2}$}

\subsection{Introduction}

Lithium-ion batteries (LIB) are a widely used power source found in many common electronic devices. LIB are comprised of an anode, cathode, and an electrolyte. In these types of batteries, lithium ions move from the negative electrode to the positive electrode during discharge and then back again when charging. Therefore it is important that the electrode material of the battery has a means of "storing" and transporting lithium ions. The c-axis channels in $\mathrm{TiO}_{2}$ rutile are large enough to accommodate interstitial $\mathrm{Li}^{+}$ ions and these channels provide a means for directional ionic conductivity. Hence, rutile is a good candidate for a LIB anode material. The anodes of conventional LIB are made of carbon, but as Kubiak et al. ${ }^{1}$ summarizes, many problems arise due to the carbonbased anode performing poorly under extreme conditions, such as low temperature and high charge/discharge rates. $\mathrm{TiO}_{2}$ has emerged as a viable candidate for an anode material because it is less expensive and safer to use than graphite-based anodes. The viability of $\mathrm{TiO}_{2}$ as a lithium intercalation material has been the subject of numerous studies. $^{2-5}$

In this chapter, I present the results of an EPR and ENDOR study of a lithiumassociated defect in $\mathrm{TiO}_{2}$ (rutile) single crystals. The defect consists of an unpaired electron trapped on a $\mathrm{Ti}^{4+}$ ion interacting with an adjacent interstitial $\mathrm{Li}^{+}$ion (i.e., $\mathrm{a} \mathrm{Ti}^{3+}$ ion next to a $\mathrm{Li}^{+}$interstitial). $\mathrm{The} \mathrm{TiO}_{2}$ crystals used in this study were purchased from Crystec. There was no evidence that interstitial lithium ions were present in the as-

received crystals. Lithium was introduced by completely surrounding the crystal with 
lithium hydroxide $(\mathrm{LiOH})$ powder in a small ceramic boat and then placing the boat in a furnace preheated to $450{ }^{\circ} \mathrm{C}$. Anneal times were on the order of several hours. Annealing separate crystals for 6 and 18 hours did not immediately affect the crystal's coloration, i.e., the crystals appeared the same before and after the anneal. After several weeks at room temperature and being exposed to ambient room light, however, the crystals turned brown. Possible reasons for this observation are discussed in the next chapter. A crystal annealed for one hour did not produce as noticeable a change in color, suggesting that the interstitial lithium ions in the crystal are responsible for the brown color that appears over time. In other words, more interstitial lithium ions lead to increased brown color.

\subsection{Sample Preparation}

The $\mathrm{TiO}_{2}$ crystal used in this study was grown by Crystec and was a c-axis plate with dimensions of $10 \times 10 \times 2 \mathrm{~mm}^{3}$. EPR-sized samples $\left(4 \times 3 \times 2 \mathrm{~mm}^{3}\right)$ were cut from this larger plate and heated in the $\mathrm{LiOH}$ powder. Lithium can be diffused into rutile through the channels that run along the $\mathrm{c}$ axis of the crystal. It is much easier to diffuse $\mathrm{Li}$ in this direction than along the other axes of the crystal. At $550^{\circ} \mathrm{C}$, the diffusion coefficient parallel to the $\mathrm{c}$ axis is about $10^{8}$ times larger than the diffusion coefficient perpendicular to the c axis. ${ }^{6}$ When preparing a sample for lithium in-diffusion, the $\mathrm{LiOH}$ powder was placed in the bottom of the ceramic boat, then the crystal was placed in the boat, and finally, additional powder was added until the crystal was completely covered. The boat was then placed in a furnace preheated to $450{ }^{\circ} \mathrm{C}$. This allowed the crystal to be in contact with the powder on all sides and increased the likelihood of lithium diffusion. 
Three different crystals were treated in this manner with annealing times of 1 hour, 6 hours, and 18 hours, respectively.

\subsection{EPR Results}

Prior to annealing in LiOH powder, no lithium-related EPR signals were observed in the Crystec sample. Signals associated with the singly and doubly ionized oxygen vacancy, the $\mathrm{Ti}^{3+}-\mathrm{Si}^{4+}$ defect, $\mathrm{Fe}^{3+}$ and $\mathrm{Cr}^{3+}$ are all visible under the photoexcitation conditions outlined in Ref. 7. An 18-hour anneal in $\mathrm{LiOH}$ powder produced the EPR signals shown in Fig. 5.1. Two are seen in this spectrum; the $\mathrm{Ti}^{3+}-\mathrm{Si}^{4+}$ signal at the center of the spectrum and the lithium interstitial signal on the high-field side near 350.5 mT. These data in Fig. 5.1 were taken at $25 \mathrm{~K}$ in order to optimize the intensity of the $\mathrm{Ti}^{3+}-\mathrm{Si}^{4+}$ signal. This $\mathrm{Ti}^{3+}-\mathrm{Si}^{4+}$ defect was initially reported by Yang et al. ${ }^{7}$ The c-axis $\mathrm{g}$ value of 1.938 in Fig. 5.1 is identical to the g value quoted in their work. The $\mathrm{Ti}^{3+}-\mathrm{Si}^{4+}$ center is formed when a $\mathrm{Ti}^{4+}$ cation next to a substitutional $\mathrm{Si}^{4+}$ ion traps an electron and becomes a $\mathrm{Ti}^{3+}$ ion. Unlike their observations, laser light is not required to produce the $\mathrm{Ti}^{3+}-\mathrm{Si}^{4+} \mathrm{EPR}$ signal in the lithium-diffused sample. This suggests that the electron is stabilized at the $\mathrm{Ti}^{4+}$ site by the interstitial $\mathrm{Li}^{+}$ion.

Several small hyperfine lines can be seen surrounding the large center line of the $\mathrm{Ti}^{3+}-\mathrm{Si}^{4+}$ spectrum. As indicated with stick diagrams, a subset of these hyperfine lines is attributed to an interaction with the titanium nuclei. The two isotopes of titanium have nearly identical magnetic moments, so the splitting from the large center line will be nearly the same for both isotopes. ${ }^{47} \mathrm{Ti}$ has a nuclear spin $\mathrm{I}=5 / 2$ and is $7.4 \%$ abundant, while ${ }^{49} \mathrm{Ti}$ has a nuclear spin $\mathrm{I}=7 / 2$ and is $5.4 \%$ abundant. Thus, there are six hyperfine 


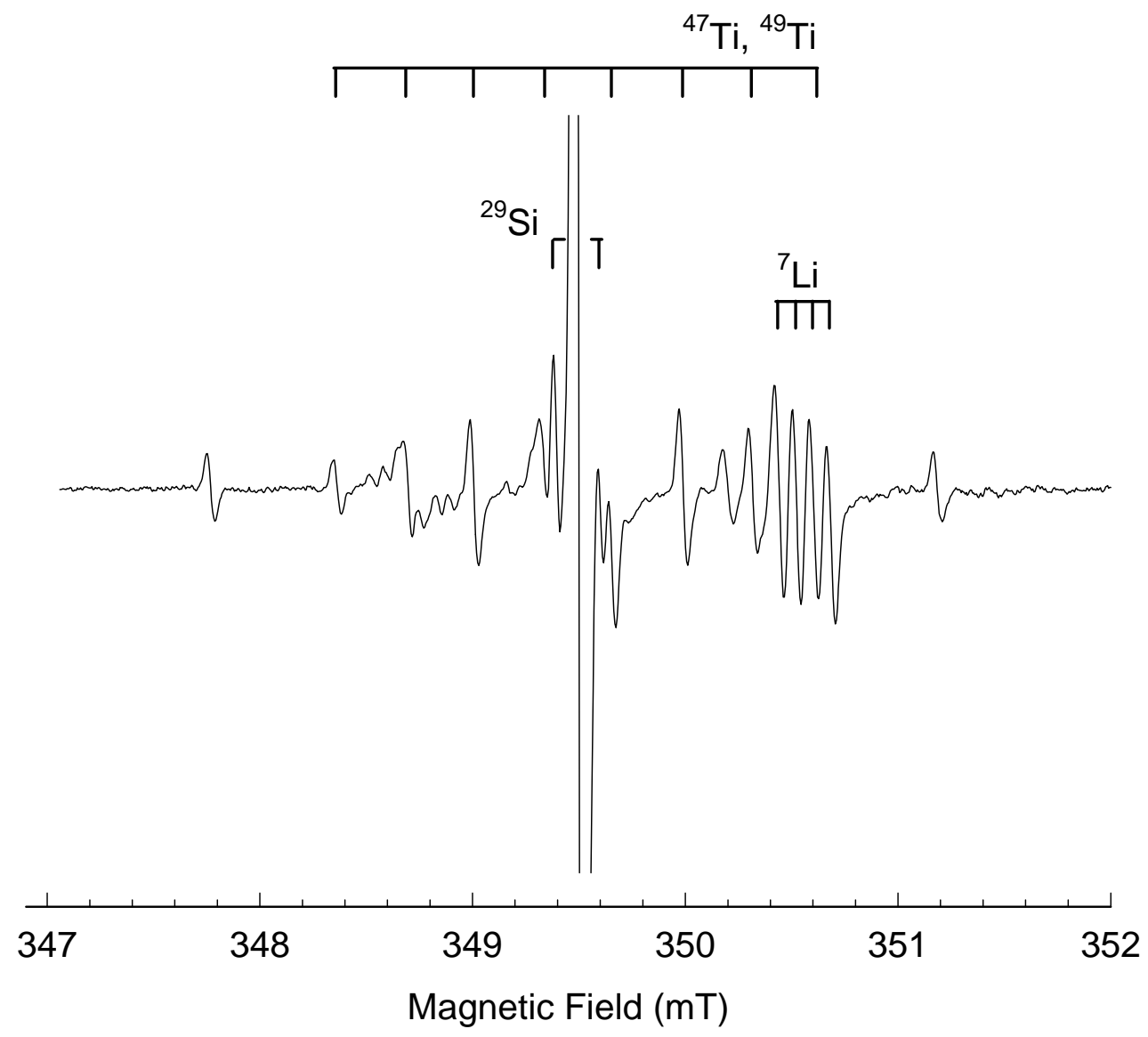

Figure 5.1. $\mathrm{Ti}^{3+}-\mathrm{Si}^{4+}$ and $\mathrm{Li}^{+}$interstitial defects in $\mathrm{TiO}_{2}$. Hyperfine lines due to ${ }^{47} \mathrm{Ti},{ }^{49} \mathrm{Ti},{ }^{29} \mathrm{Si}$, and ${ }^{7} \mathrm{Li}$ are indicated by stick diagrams. The two outermost EPR lines are unidentified. This trace was taken at $25 \mathrm{~K}$.

lines associated with ${ }^{47} \mathrm{Ti}$ and eight lines associated with ${ }^{49} \mathrm{Ti}$. Since the magnetic moments are nearly the same for the two isotopes, the pattern seen in Fig. 5.1 results; the six ${ }^{47} \mathrm{Ti}$ lines cover the inner six ${ }^{49} \mathrm{Ti}$ lines, leaving two isolated outer ${ }^{49} \mathrm{Ti}$ lines. The highest-field ${ }^{49} \mathrm{Ti}$ line is covered up by the four-line ${ }^{7} \mathrm{Li}$ signal. Two additional hyperfine lines, located very near the center line, are produced when the unpaired spin interacts with the nearest-neighbor silicon impurity. ${ }^{29} \mathrm{Si}$ has nuclear spin $\mathrm{I}=1 / 2$ and is $4.67 \%$ abundant. Hence, these two extra hyperfine lines are consistent with a silicon impurity. Lithium has two magnetic isotopes. ${ }^{6} \mathrm{Li}$ has nuclear spin $\mathrm{I}=1$ and is $7.5 \%$ abundant, 
while ${ }^{7} \mathrm{Li}$ has nuclear spin $\mathrm{I}=3 / 2$ and is $92.5 \%$ abundant. The magnetic moment of ${ }^{6} \mathrm{Li}$ is less than half the moment of ${ }^{7} \mathrm{Li}$. Therefore the hyperfine splitting due to ${ }^{6} \mathrm{Li}$ is less than that of ${ }^{7} \mathrm{Li}$, resulting in the more intense four-line ${ }^{7} \mathrm{Li}$ pattern "hiding" the weaker threeline ${ }^{6} \mathrm{Li}$ EPR signal.

Figure 5.2 focuses on a portion of Fig. 5.1, i.e., it highlights the four-line $\mathrm{Li}^{+}$ hyperfine pattern. These data in Fig. 5.2 were taken at $36 \mathrm{~K}$, the optimal temperature for monitoring the lithium signal. This signal is observed without any laser illumination, and its intensity is not affected by laser light. The signal in Fig. 5.2 was obtained from the $\mathrm{TiO}_{2}$ crystal that was annealed in $\mathrm{LiOH}$ powder for 18 hours. As expected, the intensity of the lithium signal becomes progressively larger as the annealing time is increased.

The angular dependence of the four-line lithium-associated EPR spectrum is

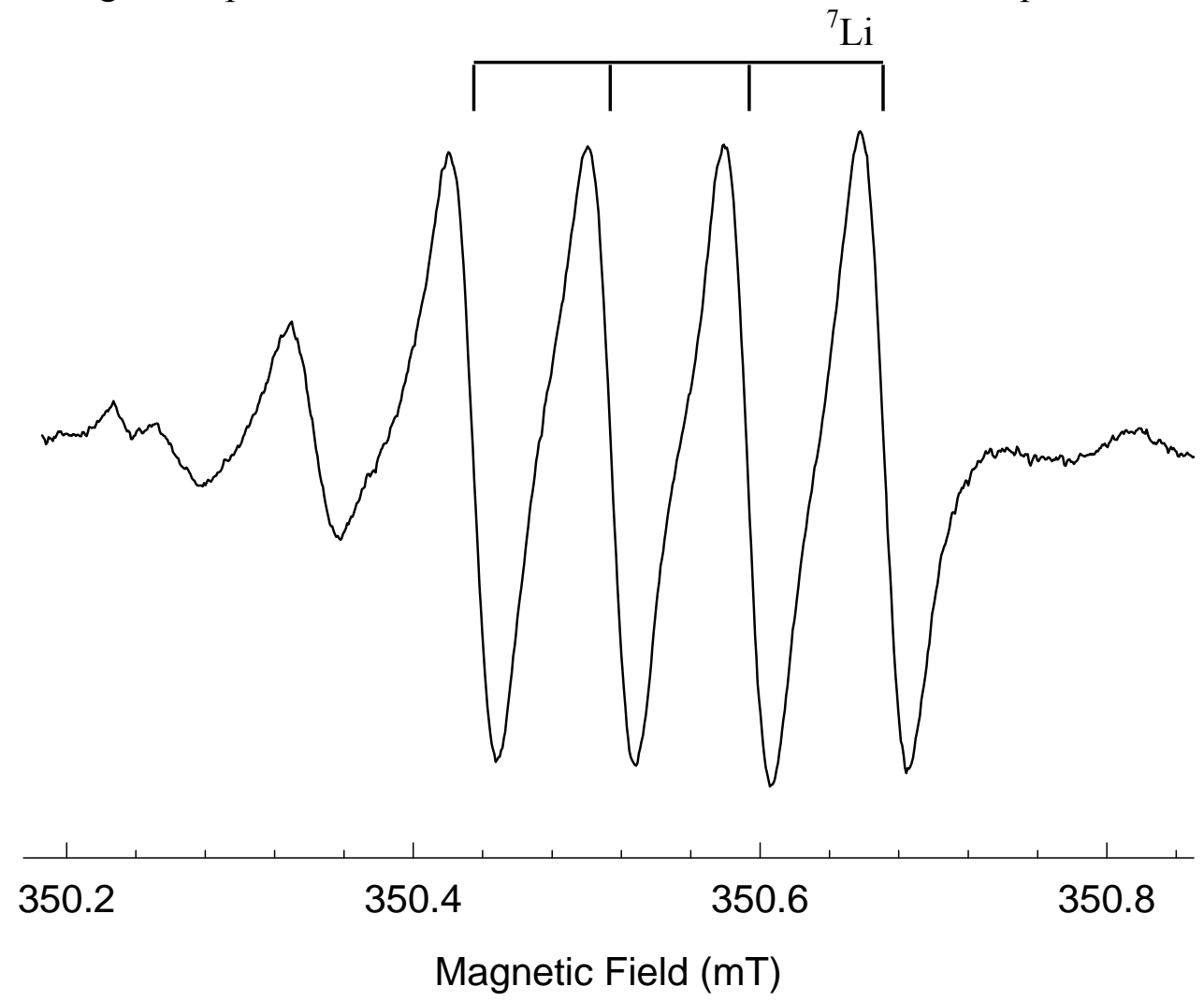

Figure 5.2. $\mathrm{Li}^{+}$-interstitial defect in $\mathrm{TiO}_{2}$. This spectrum was taken at $36 \mathrm{~K}$ to maximize the intensity of the Li signal. 
similar to the angular dependence of the neutral hydrogen donor in $\mathrm{TiO}_{2}{ }^{8}$ Trace (a) in Fig. 5.3 was taken with the field parallel to the [100] crystal axis and trace (b) in Fig. 5.3 was taken with the field rotated five degrees off of [100] toward the [110] direction in the basal plane. Two sets of four lines appear when the magnetic field is aligned along the [100] direction and along the [110] direction. Each set splits into two sets when the magnetic field is rotated in the basal plane, resulting in 16 total lines. When the field is aligned along the [110] direction, only one of the two sets of four lines is observable. The higher-field set is obstructed by the $\mathrm{Ti}^{3+}-\mathrm{Si}^{4+}$ EPR signal. A [110] spectrum is not shown here.

The angular dependence of the $\mathrm{Li}^{+}$EPR signal in the basal plane supports the assignment of this four-line EPR signal to an interstitial $\mathrm{Li}^{+}$ion. A defect consisting of a $\mathrm{Li}^{+}$interstitial located in the c-axis channel with a nearest-neighbor substitutional $\mathrm{Ti}^{3+}$ ion has two magnetically inequivalent orientations (i.e., sites) when the field is rotated from [001] to [100] or from [001] to [110]. There are, however, four magnetically inequivalent sites when the magnetic field is rotated in the basal plane. On the other hand, a substitutional defect on an unperturbed $\mathrm{Ti}^{4+}$ lattice site has only two magnetically inequivalent orientations for field alignment in any direction, since the one $\mathrm{Ti}^{4+}$ site is rotated 90 degrees from the other. As seen in Fig. 5.3, the $\mathrm{Li}^{+}$and $\mathrm{Ti}^{3+}-\mathrm{Si}^{4+}$ defects behave differently as the magnetic field is rotated. The $\mathrm{Li}^{+}$signal behaves as an interstitial, while the $\mathrm{Ti}^{3+}-\mathrm{Si}^{4+}$ center follows the pattern of an isolated substitutional defect. 

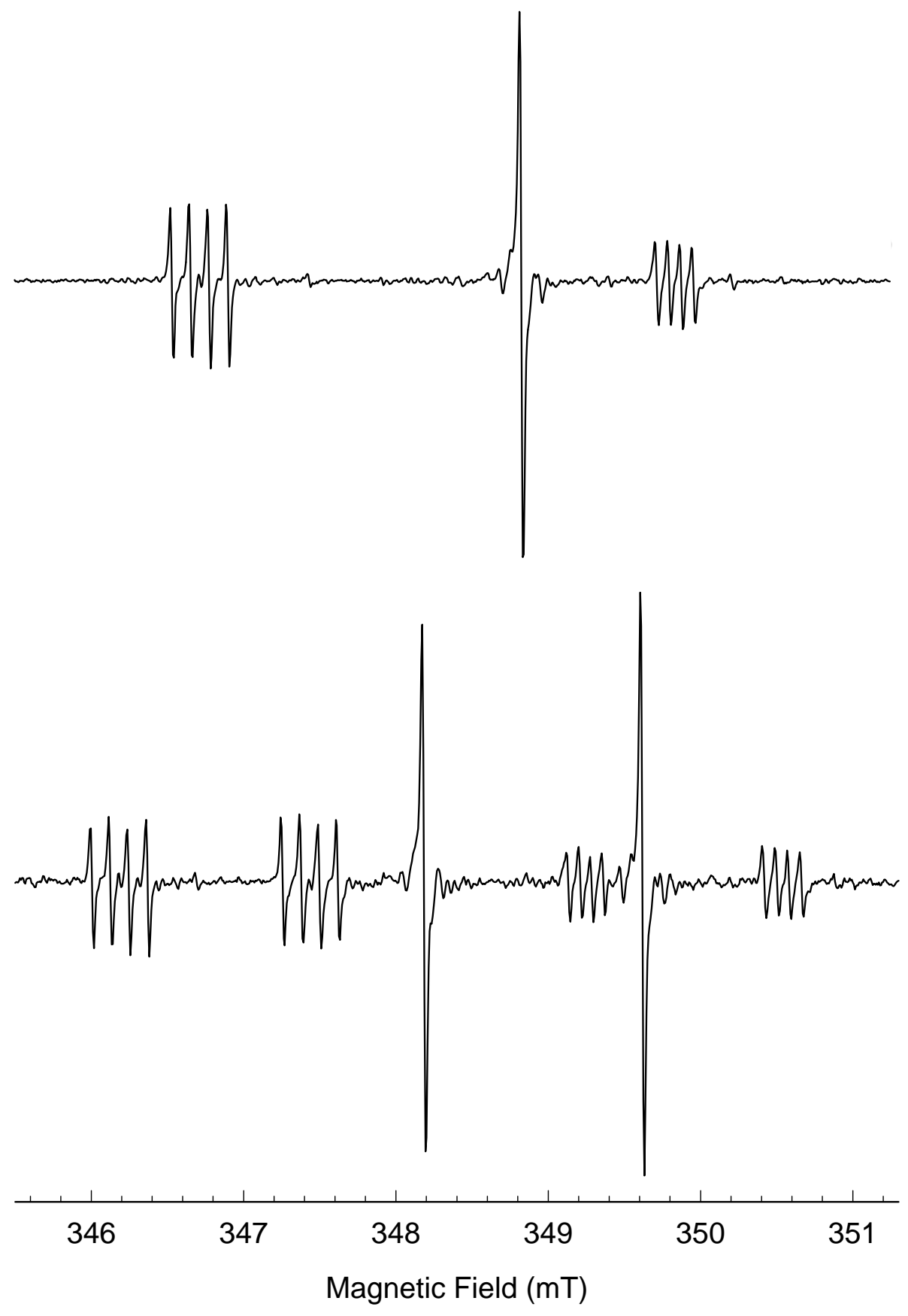

Figure 5.3. Effect of magnetic field rotation in the basal plane.

Trace (a) was taken with the field aligned along [100] and trace (b) was taken when the field was rotated 5 degrees off of [100] in the basal plane. The sample temperature was $36 \mathrm{~K}$. 


\subsection{ENDOR Study}

An ENDOR experiment was performed on the four-line $\mathrm{Li}^{+} \mathrm{EPR}$ spectrum in order to verify that the hyperfine structure is indeed due to a lithium nucleus. Figure 5.4 is an ENDOR spectrum taken with the magnetic field aligned along the [001] direction and fixed at 3524.2 G, corresponding to the second-lowest of the four EPR lines in Fig. 5.2. The resulting ENDOR spectrum, shown in Fig. 5.4, consists of two lines. Although these same two lines appear when the magnetic field is fixed on any one of the four EPR lines, fixing the magnetic field on the second lowest line gives the most intense ENDOR lines.

The separation between the EPR lines within a set of four corresponds to the hyperfine parameter A. From Fig. 5.2, it is about $0.78 \mathrm{G}$, or $2.11 \mathrm{MHz}$ at $\mathrm{g}=1.932$.

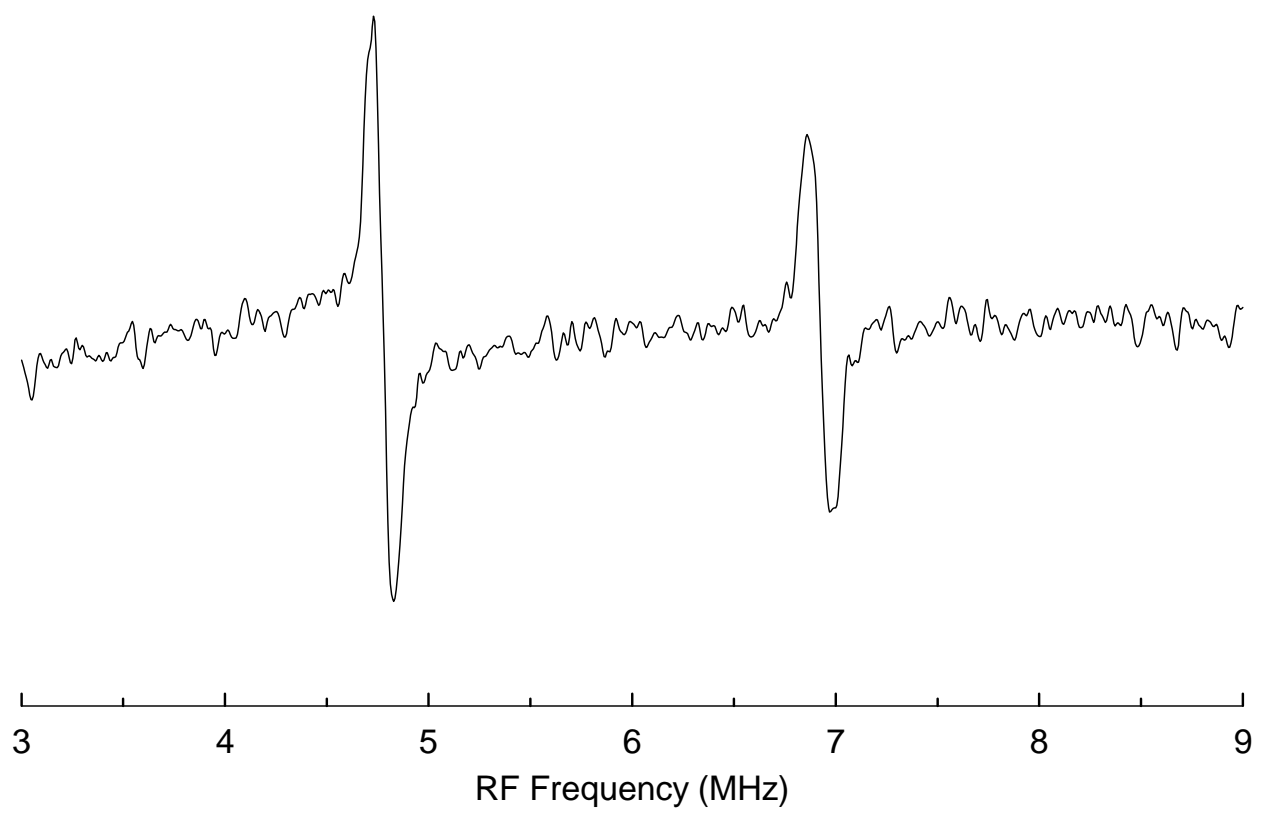

Figure 5.4. ENDOR spectrum of the $\mathrm{Li}^{+}$interstitial with the field aligned along the [001] axis. The magnetic field was fixed on the second-lowest EPR line. The microwave frequency was 9.532165 MHz. Sample temperature was $20 \mathrm{~K}$. 
The Larmor frequency, or "free spin" frequency, of a ${ }^{7} \mathrm{Li}$ nucleus is $5.83 \mathrm{MHz}$ at 3524.2 G. Therefore, $v_{\mathrm{n}}>\mathrm{A} / 2$, meaning that the two ENDOR lines should be centered on $v_{\mathrm{n}}$ and separated by A. The two lines in Fig. 5.4 appear at 4.78 MHz and 6.93 MHz. Their separation of $2.14 \mathrm{MHz}$ agrees well with the EPR hyperfine splitting, while the center position of $5.87 \mathrm{MHz}$ is in good agreement with $v_{\mathrm{n}}$ for a ${ }^{7} \mathrm{Li}$ nucleus.

As mentioned in the preceding paragraph, the same two ENDOR lines appear when fixing the magnetic field on any of the four EPR lines. I note that ${ }^{7} \mathrm{Li}$, with $\mathrm{I}=3 / 2$, has a non-zero nuclear electric quadrupole moment. In principle, additional ENDOR lines should appear, with their positions governed by the magnitude of the nuclear quadrupole coupling parameter $\mathrm{P}$. The most plausible explanation for the absence of additional lines is that the $\mathrm{P}$ value is sufficiently small that the additional lines are not resolved in Fig. 5.4.

\subsection{Spin-Hamiltonian Analysis}

Figure 5.5 shows the EPR angular dependence of the $\mathrm{Li}^{+}$interstitial defect in all three high-symmetry planes. The open circles represent experimental data points. These data points are the average line position of the four EPR lines within each set. The solid lines are computer-generated using the "best" parameters for the g matrix, obtained from a least squares fitting procedure similar to the one in Appendix A.1

The following spin Hamiltonian was used to determine the principal values and principal axis directions of the $\mathrm{g}$ matrix for the $\mathrm{Li}^{+}$interstitial defect.

$$
\hat{\mathrm{H}}=\mu_{\mathrm{B}} \vec{S} \cdot \vec{g} \cdot \vec{B}
$$

Input data for the fitting routine were 13 magnetic field values and their corresponding 


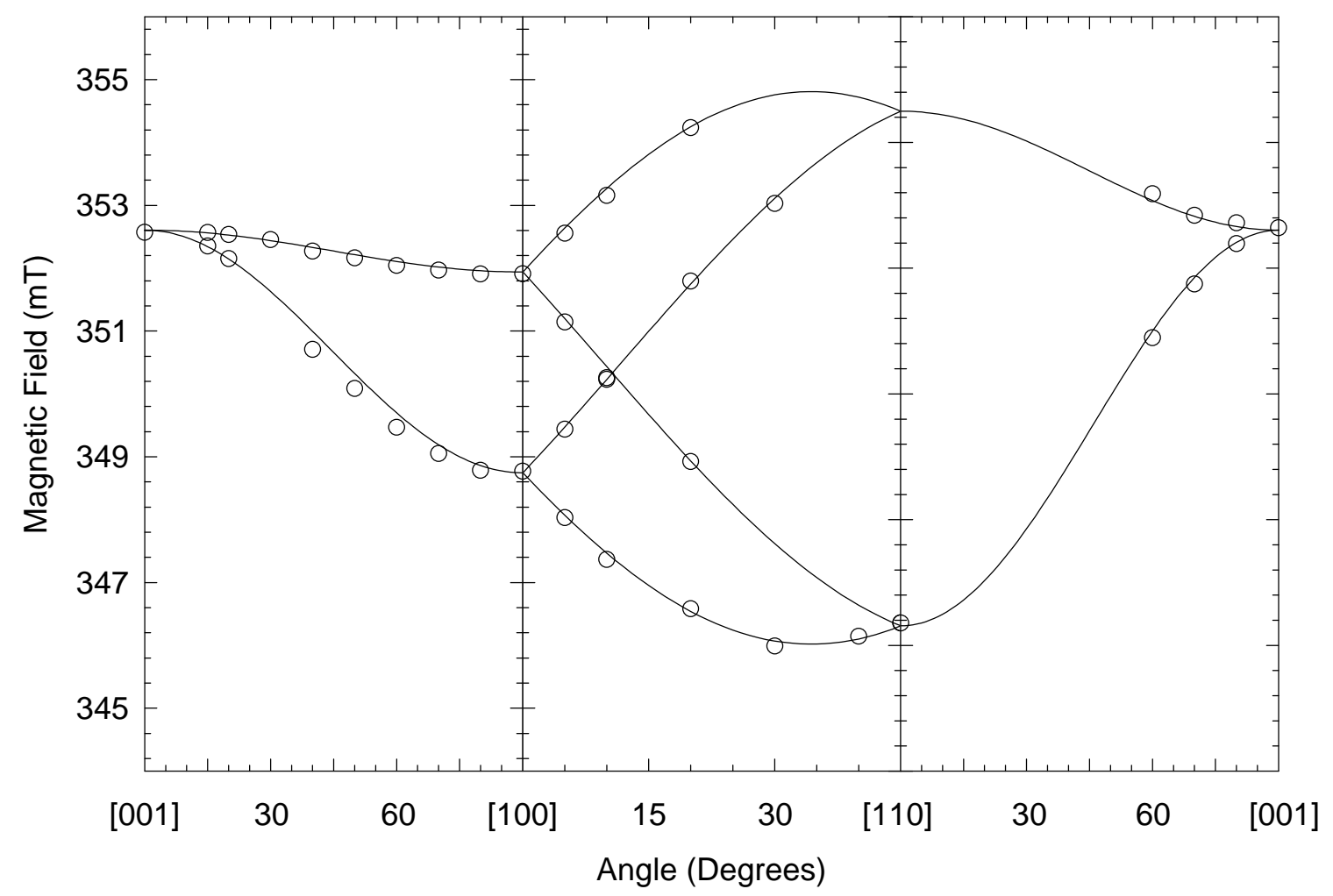

Figure 5.5. Angular dependence of the $\mathrm{Li}^{+}$interstitial in all three high-symmetry planes. Solid lines represent computer-generated best lines determined by using the values in Table 1 . The circles are the average of the four EPR resonance fields in each set. The lines were generated at $9.535935 \mathrm{MHz}$ and the line positions of the experimental data were adjusted accordingly.

microwave frequencies. Additional data points were taken and included Fig. 5.5, but were not used in the fitting procedure. The solid lines were generated using the data in

Table 5.1. Principal values and principal axis directions for the $g$ matrix of the $\mathrm{Li}^{+}$interstitial in rutile.

\begin{tabular}{ccc}
\hline Principal Values & $\begin{array}{c}\text { Principal Axis } \\
\text { Direction }\end{array}$ \\
\hline \hline g matrix & \pm 0.0002 & \\
$\mathrm{~g}_{1}$ & 1.9690 & $10.57^{\circ}$ from $[110]$ \\
$\mathrm{g}_{2}$ & 1.9202 & $10.57^{\circ}$ from $[\overline{1} 10]$ \\
$\mathrm{g}_{3}$ & 1.9322 & {$[001]$} \\
\hline
\end{tabular}


Table 5.1. The Euler angles are defined using the "zxz" convention outlined in Appendix B.

\subsection{Defect Model}

The principal $g$ values of the four-line lithium signal are close to the $g$ values of other defects that are associated with $\mathrm{Ti}^{3+}$ ions in $\mathrm{TiO}_{2}{ }^{7-9}$ In general, $\mathrm{Ti}^{3+}$ EPR signals are observed in $\mathrm{TiO}_{2}$ when $\mathrm{Ti}^{4+}$ ions trap an electron and convert to $\mathrm{Ti}^{3+}$ at low temperature. This unpaired spin at the $\mathrm{Ti}^{3+}$ ion interacts with adjacent nuclei and hence EPR hyperfine patterns associated with these adjacent nuclei give information about the defect model. A lithium atom $\left(\mathrm{Li}^{0}\right)$ is easily ruled out as the responsible defect for the four-line lithium spectrum in Fig. 5.2. $\mathrm{Li}^{0}$ has one unpaired electron that occupies an $\mathrm{s}$ orbital. An s-orbital electron would exhibit no EPR angular dependence and have an isotropic hyperfine splitting of $143.4 \mathrm{G}^{10}{ }^{10}$ The observed splittings between the four lines in Fig. 5.2 are $\sim 0.70 \mathrm{G}$, much weaker than expected for a $\mathrm{Li}^{0}$ atom. Therefore, $\mathrm{a} \mathrm{Li}^{+}$ion is the adjacent defect responsible for the four-line EPR signal in Figs. 5.1 and 5.2.

Lithium in $\mathrm{TiO}_{2}$ is not expected to occupy a substitutional site due to the large discrepancy between the valence state of the host cation $\left(\mathrm{Ti}^{4+}\right)$ and the valence state of a $\mathrm{Li}^{+}$ion. The basal plane angular dependence, where four distinct orientations of the defect are observed, verifies that $\mathrm{Li}^{+}$exists in $\mathrm{TiO}_{2}$ as an interstitial ion located within the c-axis channels. Figure 5.6 shows a schematic diagram of the $\mathrm{Li}^{+}$interstitial site. This figure is a projection onto the $\mathrm{c}$ plane and illustrates how the $\mathrm{Li}^{+}$ion is arranged in the $\mathrm{c}-$ channel. There are two planes of atoms in the figure; the atoms labeled with subscript ' 1 ' line in a plane below the atoms with subscript ' 2 '. The interstitial atom lies in the upper 
plane, the same as the $\mathrm{Ti}^{3+}$ ion. The fact that the [001] axis is a principal axis direction provides evidence that the $\mathrm{Li}^{+}$ion lies in the same plane as the center titanium ion in the rutile unit cell. In Fig. 5.6, this ion is assigned a $\mathrm{Ti}^{3+}$ valence state. The principal axis associated with $\mathrm{g}_{1}$ lies $10.57^{\circ}$ from the [110] direction, and is assigned to point toward the $\mathrm{Li}^{+}$ion. The direction of $\mathrm{g}_{1}$ indicates that the $\mathrm{Li}^{+}$ion lies closer to the $\mathrm{Ti}^{3+}$ ion, rather than in the middle of the channel.

Stashans et al. ${ }^{3}$ suggests two possible interstitial locations, the $(1 / 2,0,1 / 2)$ lattice site and the $(0,0,1 / 2)$ lattice site. I rule out the $(0,0,1 / 2)$ site in the unit cell because, when considering the ionic radii of oxygen, titanium, and lithium, there is very little

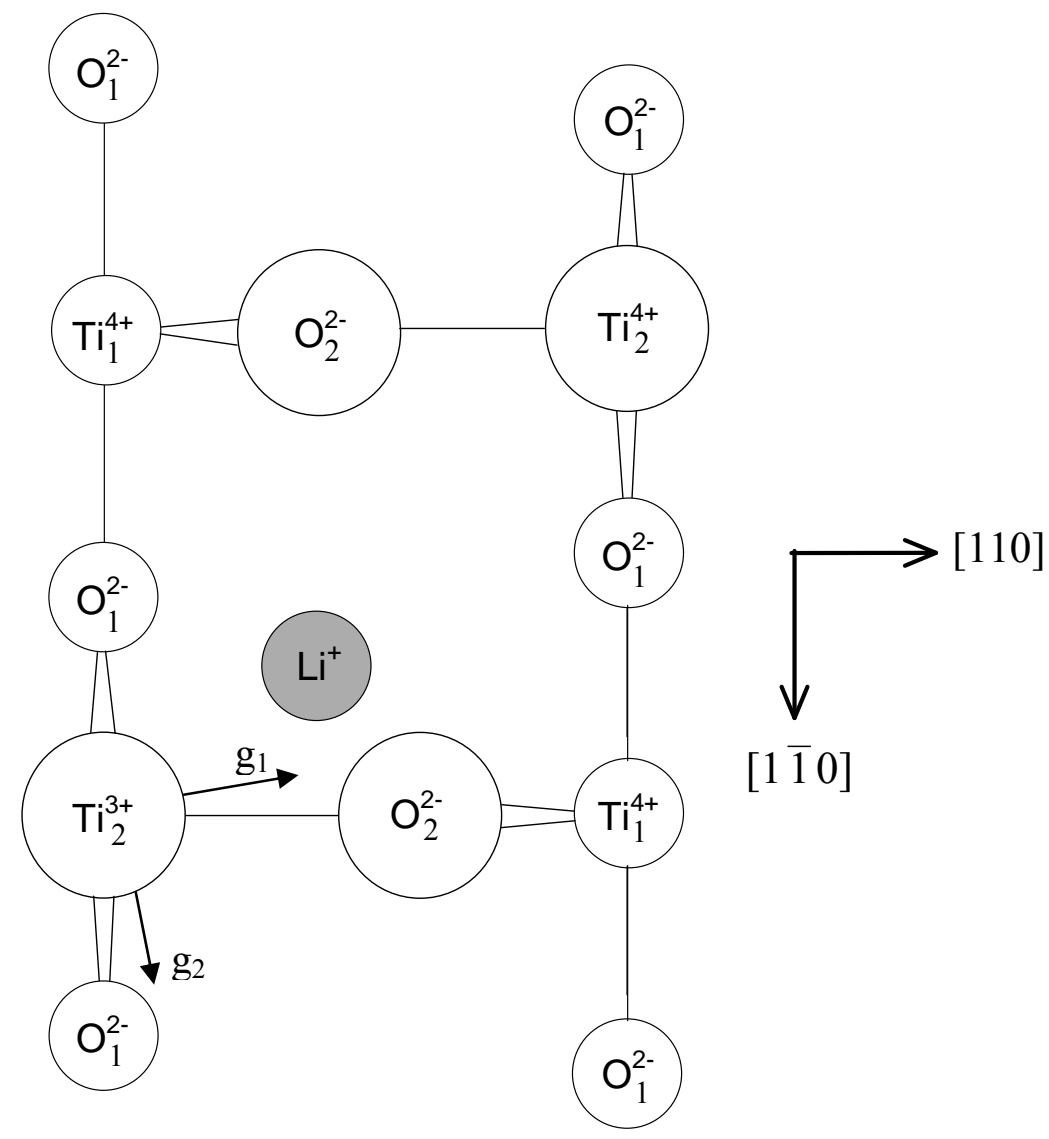

Figure 5.6. Location of the $\mathrm{Li}^{+}$interstitial in rutile and the $\mathrm{g}_{1}$ and $\mathrm{g}_{2}$ principal axes. Note the titanium ion in the lower left portion of the sketch is $\mathrm{Ti}^{3+}$, while the others are $\mathrm{Ti}^{4+}$. 
space available at the $(0,0,1 / 2)$ lattice site for the $\mathrm{Li}^{+}$ion to occupy. The principal axis directions of the g matrix indicate that the location of the $\mathrm{Li}^{+}$ion within the channel lies in the same plane as the $\mathrm{Ti}^{3+}$ ion, but not at the $(1 / 2,0,1 / 2)$ site in the unit cell (i.e., the center of a c-axis channel). Given that the direction of $g_{1}$ is only $10.57^{\circ}$ from the [110] direction, I propose that the $\mathrm{Li}^{+}$ion lies closer to the $\mathrm{Ti}_{2}^{3+}$ and the $\mathrm{O}_{2}^{2-}$ ion in Fig. 5.6, rather than in the middle of the channel. This conclusion is further supported on the grounds of Coulombic attraction. The $\mathrm{Ti}^{3+}$ ion is effectively a negative charge, and so the positively charged $\mathrm{Li}^{+}$ion would naturally be attracted to the negative $\mathrm{Ti}^{3+}$ ion. The negatively charged $\mathrm{O}^{2-}$ ions nearby would also facilitate attraction of the $\mathrm{Li}^{+}$ion toward the $\mathrm{Ti}^{3+}$ ion. A complete hyperfine matrix would provide confirmation as to the location of the interstitial $\mathrm{Li}^{+}$ion within the $\mathrm{c}$ channel. The hyperfine matrix is not worked out here because ENDOR signals were not observable when the magnetic field was aligned off the $\mathrm{c}$ axis. 


\section{Chapter 5 References}

1. P. Kubiak, M. Pfanzelt, J. Geserick, U. Hörmann, N. Hüsing, U. Kaiser, and M.Wohlfahrt-Mehrens, Journal of Power Sources 194, 1099 (2009).

2. A. R. Armstrong, G. Armstrong, J. Canales, R. Garcia, and P. G. Bruce, Advanced Materials 17, 862 (2005).

3. A. Stashens, S. Lunell, R. Bergstrom, A. Hagfeldt, and S.-E. Lindquist, Phys. Rev. B 53, 159 (1996).

4. M. V. Koudriachova, N. M. Harrison, and S. W. de Leeuw, Phys. Rev. Lett 86, 1275 (2001).

5. G. Nuspl, K. Yoshizawa, and T. Yamabe, J. Mater. Chem 7, 2529 (1997).

6. O. W. Johnson, Phys. Rev. 136, A284 1964.

7. Shan Yang, L. E. Halliburton, A. Mannivannan, P. H. Bunton, D. B. Baker, M. Klemm, S. Horn, and A. Fujishima, App. Phys. Lett. 94, 162114 (2009).

8. A. T. Brant, Shan Yang, N. C. Giles, and L. E. Halliburton, J. Appl. Phys. 110, $053714(2011)$.

9. Shan Yang, and L. E. Halliburton Phys. Rev. B 81, 035204 (2010).

10. J. R Bolton, and J. A. Weil. Electron Paramagnetic Resonance: Elementary Theory and Practical Applications. John Wiley \& Sons, 2007. 


\section{Chapter 6}

\section{Interstitial $\mathrm{Li}^{+}$Ions Adjacent to Substitutional $\mathrm{Fe}^{3+}$ Ions}

\subsection{Introduction}

In this chapter, I describe a previously unreported four-line, lithium-associated EPR signal that appears in $\mathrm{TiO}_{2}$ (rutile) crystals containing $\mathrm{Fe}^{3+}$ ions after they have been held at high temperature in the presence of lithium hydroxide $(\mathrm{LiOH})$ powder. These signals appear at much lower magnetic field (higher g value) than the isolated, interstitial lithium defect studied in the previous chapter. This new signal is assigned to an interstitial lithium ion located adjacent to a substitutional $\mathrm{Fe}^{3+}$ ion. In support of this assignment, I note that the EPR signal from this $\mathrm{Fe}^{3+}-\mathrm{Li}^{+}$defect is much more intense in crystals doped with iron.

$\mathrm{TiO}_{2}$ crystals from two sources were included in this study. One source of undoped crystals was CrysTec. In these crystals, iron was unintentionally incorporated into the lattice during growth. Dr. Satoshi Watauchi at the University of Yamanashi in Japan was the source of crystals doped with $\mathrm{Fe}^{3+}$ ions. These latter crystals were doped with iron (on the order of $10 \mathrm{ppm}$ ). In the remainder of this chapter, I will refer to samples from these sources as "CrysTec" and "Japanese". Two Crystec crystals were annealed in $\mathrm{LiOH}$ powder at $450{ }^{\circ} \mathrm{C}$ for 6 and 18 hours, respectively. One Japanese sample was annealed for six hours at $450{ }^{\circ} \mathrm{C}$.

\subsection{EPR and ENDOR Results}

As shown in Fig. 6.1, the intensity of the EPR signal from the iron-related lithium interstitial defect depends on the amount of iron present in the crystal. Trace (a) was 
taken from a Japanese sample, and trace (b) was taken from a CrysTec sample. Both of these crystals were annealed for six hours in $\mathrm{LiOH}$ powder. The signal at higher field in both traces is due to isolated $\mathrm{Fe}^{3+}$ ions ${ }^{1-3}$. This assignment is made based on the observed hyperfine splitting. ${ }^{57} \mathrm{Fe}$ is a $2.15 \%$ abundant nucleus with $\mathrm{I}=1 / 2$, which gives two hyperfine lines centered around a much large singlet. The two hyperfine lines are indicated in Fig. 6.1.

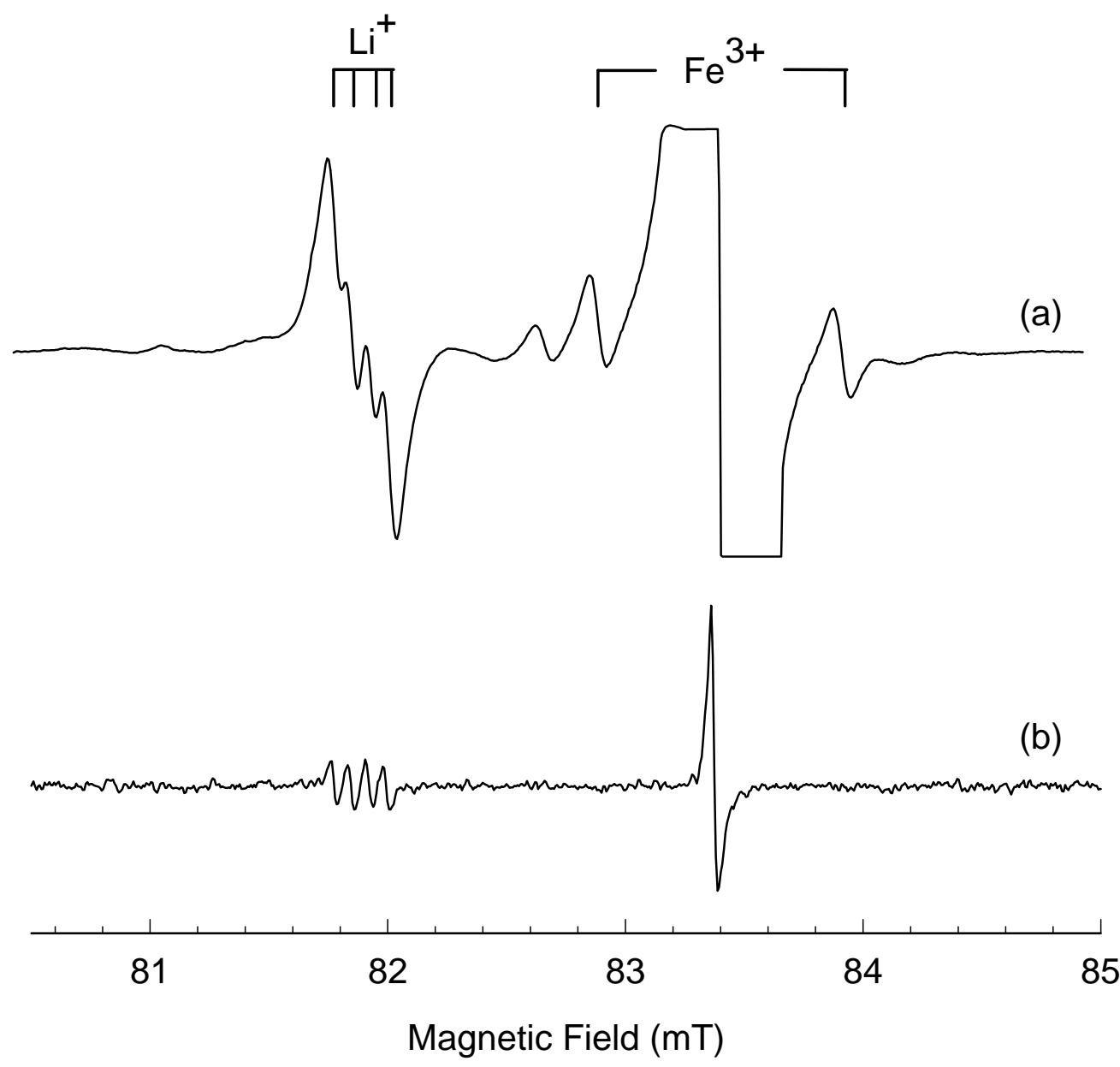

Figure 6.1. EPR spectra of a $\mathrm{Li}^{+}$ion next to an $\mathrm{Fe}^{3+}$ defect in a Japanese (a) and an as-received Crystec (b) $\mathrm{TiO}_{2}$ crystal. Spectrum (b) was taken with $447 \mathrm{~nm}$ light on the sample and the vertical scale is 10 times smaller than in (a). Spectrum (a) was taken with no light on the sample. The sample temperature was $5 \mathrm{~K}$ in both cases and the magnetic field was aligned along the $[001]$ axis. 
The four-line signal at lower field is a new spectrum which I assign to an interstitial $\mathrm{Li}^{+}$ion adjacent to a substitutional $\mathrm{Fe}^{3+}$ ion. The signal is assigned based on the fact that there are four equally intense hyperfine lines. The $92.5 \%$ abundant ${ }^{7} \mathrm{Li}$ nucleus, with $I=3 / 2$, is consistent with this description.

In order to see the lithium-associated EPR signal in the Crystec sample, the sample had to be illuminated with laser light (both 442 and $447 \mathrm{~nm}$ wavelengths are effective). The spectrum in Fig. 6.1(b) was taken after an exposure to $447 \mathrm{~nm}$ light for one second. Light is not required to see the lithium signal in Fig 6.1(a), and light does not affect the signal intensity. Both spectra were taken at $5 \mathrm{~K}$ with the magnetic field aligned along the [001] axis. The model of an interstial $\mathrm{Li}^{+}$ion adjacent to a substitutional $\mathrm{Fe}^{3+}$ ion is based on charge compensation requirements. Specifically, a substitutional $\mathrm{Fe}^{3+}$ ion needs a +1 charge nearby to compensate for the $\mathrm{Ti}^{4+}$ ion being replaced. Therefore, the lithium ion participating in this defect center is in the +1 charge state.

Figure 6.2 shows an ENDOR spectrum taken using a Crystec sample that was annealed for 18 hours. The magnetic field was fixed on the second-lowest of the four EPR lines in the $\mathrm{Li}^{+}$EPR signal. These two ENDOR lines appeared regardless of which one of the four EPR lines the magnetic field was fixed at and no additional ENDOR lines ever appeared. There is not resolved quadrupole splitting

The two ENDOR lines in Fig. 6.2 are at $3.35 \mathrm{MHz}$ and $5.55 \mathrm{MHz}$. This gives a separation of $2.2 \mathrm{MHz}$ and a center frequency of $4.45 \mathrm{MHz}$. The separation and center frequency agree favorably with twice the free spin of ${ }^{7} \mathrm{Li}$ and half the observed hyperfine splitting, respectively. The observed hyperfine splitting between the individual EPR lines 


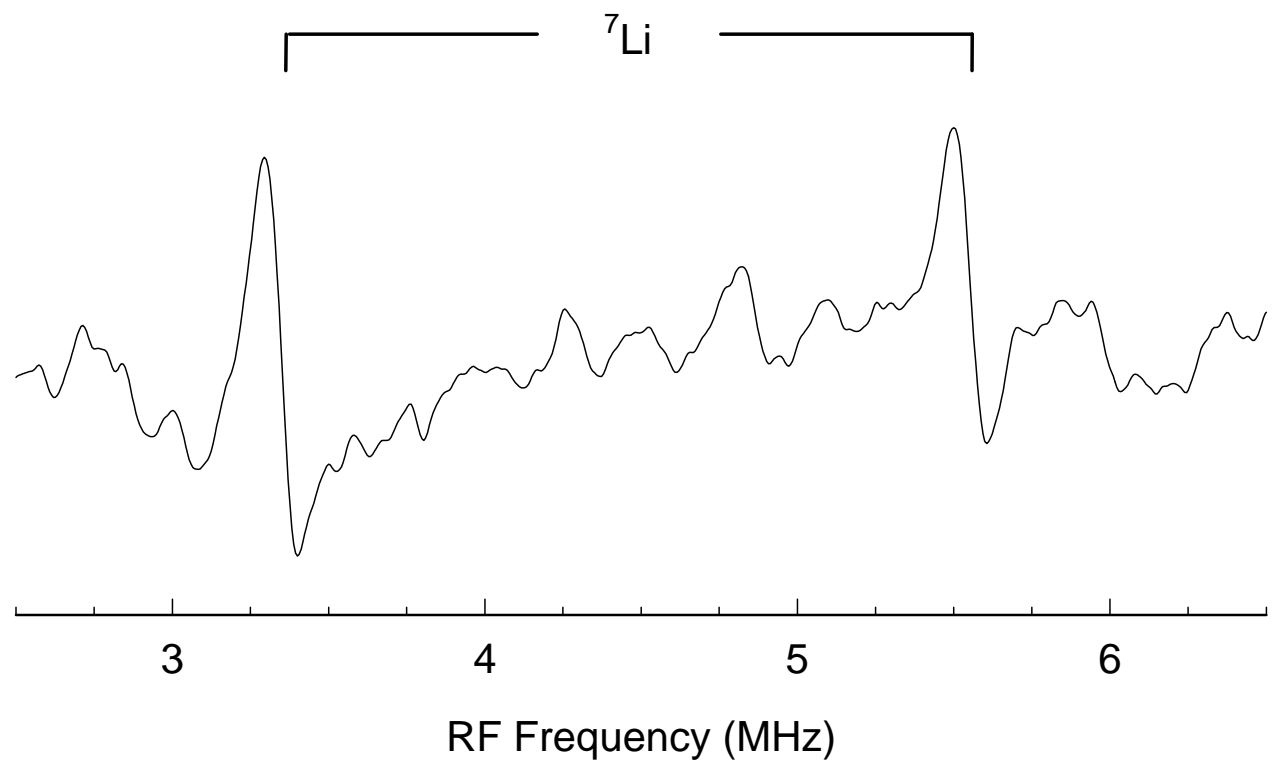

Figure 6.2. [001] axis ENDOR spectrum of $\mathrm{Li}^{+}$ions next to $\mathrm{Fe}^{3+}$ defects in a Crystec sample annealed for 6 hours. This spectrum was taken at $4 \mathrm{~K}$. The microwave frequency was $9.548768 \mathrm{GHz}$.

is 4.25 MHz and $v_{\mathrm{N}}=1.35 \mathrm{MHz}$ at the magnetic field range shown in Fig 6.1. These observations show conclusively that the four-line spectrum in Fig. 1 is due to ${ }^{7} \mathrm{Li}$.

\subsection{Photoinduced Effects}

Figure 6.3 shows three traces taken using the CrysTec crystal. Two different Crystec crystals were used to acquire Fig. 6.3, each having been cut from the same larger boule. The spectrum in 6.3(a) was acquired using a crystal that had not been heating in $\mathrm{LiOH}$ powder and was not exposed to laser light. Figures 6.3(b) and 6.3(c) were taken using a crystal that was heating in $\mathrm{LiOH}$ for 18 hours. The spectrum in 6.3(b) was taken after the sample had been placed in the microwave cavity without being exposed to light at low temperature. Figure 6.3(c) was taken after the sample was exposed to $447 \mathrm{~nm}$ laser light for one second. 

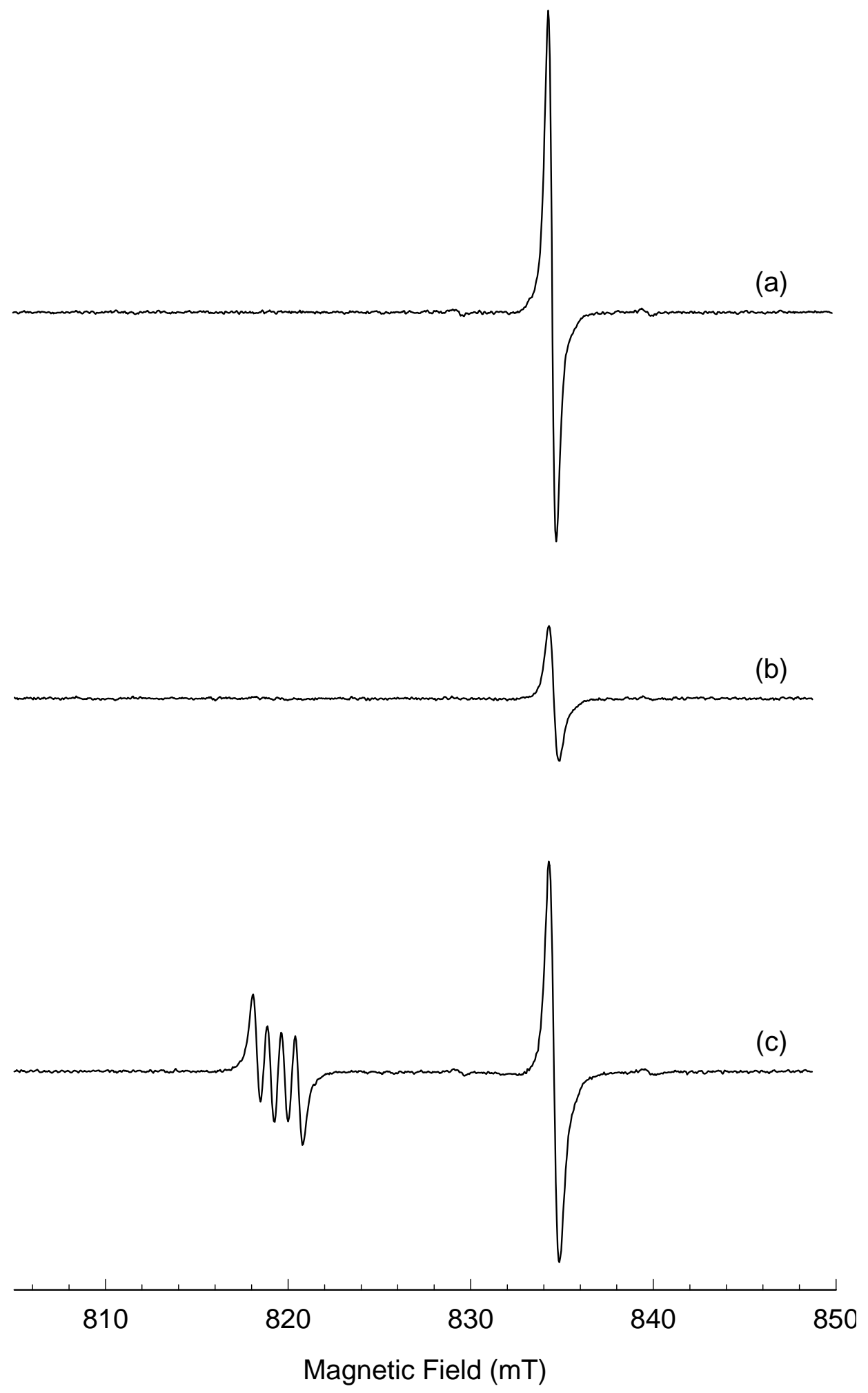

Figure 6.3. Photoinduced $\mathrm{Li}^{+}$and $\mathrm{Fe}^{3+}$ EPR spectra at $5 \mathrm{~K}$. Trace (a) was taken prior to heating in $\mathrm{LiOH}$ and without laser illumination. Traces (b) and (c) were after heating in $\mathrm{LiOH}$ for 18 hours. Trace (b) was taken before laser illumination and (c) was taken after the sample was illuminated with $447 \mathrm{~nm}$ light for one second. The magnetic field was aligned along the [001] axis. 
When the sample is cooled in the dark (i.e., placed in the helium flow through the microwave cavity without being exposed to light), the isolated $\mathrm{Fe}^{3+} \mathrm{EPR}$ signal is observed at $5 \mathrm{~K}$ in both annealed and unannealed crystals. One can see that the intensity of the $\mathrm{Fe}^{3+}$ EPR signal is reduced by more than $50 \%$ after heating the sample in $\mathrm{LiOH}$ powder. This means that the iron defect in the crystal has converted to a different charge state as a result of the annealing process. Illuminating the sample with $447 \mathrm{~nm}$ light for one second results in an increase in the $\mathrm{Fe}^{3+}$ signal intensity as well as the appearance of the four-line lithium signal.

These signals gradually decrease in intensity over periods of tens of seconds when the laser is left on the sample. At $5 \mathrm{~K}$, the EPR signal intensity remains constant for many minutes after the laser is shuttered. The $\mathrm{Li}^{+} \mathrm{EPR}$ signal is not observable at $5 \mathrm{~K}$ after warming the sample to $25 \mathrm{~K}$ for a few seconds The behavior of the $\mathrm{Fe}^{3+}$ signal in this experiment is in direct contrast with the behavior of this signal is as-received CrysTec crystals. The $\mathrm{Fe}^{3+}$ EPR signal decreases by approximately $40 \%$ when an asreceived crystal is illuminated with $447 \mathrm{~nm}$ laser light at $5 \mathrm{~K}$. As discussed in Chapter 4, holes created by laser illumination were trapped at $\mathrm{Fe}^{3+}$ ions in as-received crystals, converting them to $\mathrm{Fe}^{4+}$ ions. This experiment suggests that either an $\mathrm{Fe}^{4+}$ or an $\mathrm{Fe}^{2+}$ ion is converted to an $\mathrm{Fe}^{3+}$ ion when there is an adjacent $\mathrm{Li}^{+}$interstitial ion.

The appearance of the four-line spectrum in trace 6.3(b) is accompanied by the appearance of three additional centers at higher magnetic field, as shown in Figure 6.4. The less intense signals in Fig. 6.4 are part of a six-line set that is assigned to an $\mathrm{Al}^{3+}$ hole center. Aluminum is a substitutional defect, replacing a $\mathrm{Ti}^{4+}$ in the lattice. Charge compensation is fulfilled when a hole is trapped on an adjacent oxygen ion. Aluminum 


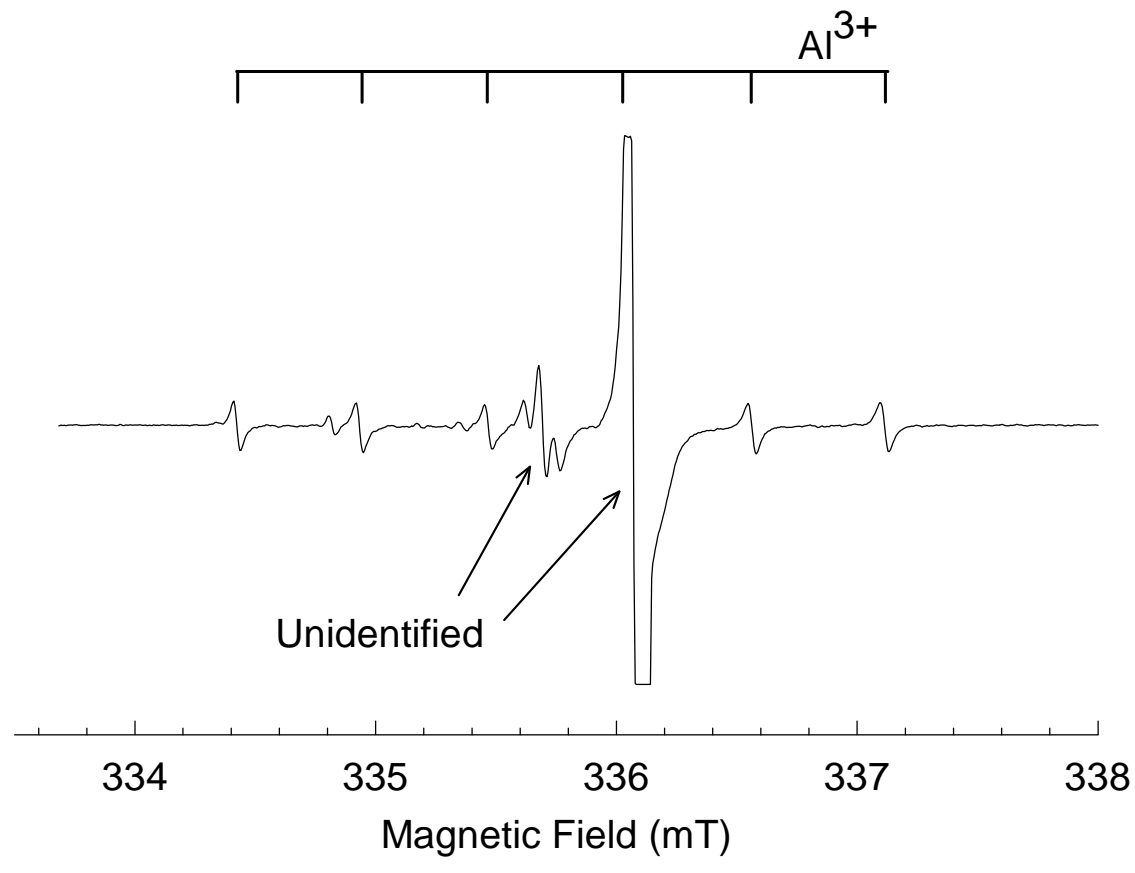

Figure 6.4. Photoinduced $\mathrm{Al}^{3+}$ center at $5 \mathrm{~K}$. This spectrum was taken with $447 \mathrm{~nm}$ laser light on and is not observed in an as-received Crystec sample.

is a $100 \%$ abundant nucleus with $I=5 / 2$. This six-line EPR signal was first reported by Zwingel in $1976{ }^{4}$ The $g$ value of the large singlet at $3360 \mathrm{G}$ in this spectrum is in close, but not exact agreement with the photoinduced, self-trapped hole center reported by Yang, Brant, and Halliburton. ${ }^{5}$ At this time, however, there is not definitive proof that the large singlet in Fig. 6.4 is the self-trapped hole center. The three-line signal at 3357 $\mathrm{G}$ is unidentified. It is most likely a hole trap since its $\mathrm{g}$ value is greater than that of a free electron $\left(\mathrm{g}_{\mathrm{e}}\right)$. The self-trapped hole can be seen as reported by Yang et al. in asreceived CrysTec crystals, but the three-line signal has not been observed in as-received crystals. The six-line set in Fig. 6.4 assigned to $\mathrm{Al}^{3+}$ is not seen with laser illumination in either an as-received Crystec crystal or the Japanese crystal.

The centers in Fig. 6.4 cannot be seen at all without laser light, and their 
intensities decrease by approximately $50 \%$ when the light is removed. There are also light-induced signals from hydrogen-related defects that appear with laser light (not shown). One of these signals corresponds to the hydrogen donor discussed in Chapter 3. Some of these signals also disappear when the light is removed, directly accompanying the decrease in $\mathrm{Al}^{3+}$ signal intensity. This indicates that the electrons trapped by hydrogen are not stable and recombine with some of the holes trapped by $\mathrm{Al}^{3+}$ ions.

There is an interesting difference in the behavior of the $\mathrm{Fe}^{3+}$ and $\mathrm{Li}^{+}$signals when the sample is exposed to laser light depending on how long after the LiOH heating one observes these signals. Immediately after taking the sample out of the heated powder, the color of the crystal is not noticeably different than that of an as-received crystal; they are opaque and yellowish in color. Several weeks after heating, the crystal, to the eye, becomes brown in color. All of the data presented in this chapter was taken on a crystal that had been exposed to room light for over one month, and were brown. When one studies an annealed crystal one day after the heating treatment, the behavior of the $\mathrm{Fe}^{3+}$ and $\mathrm{Li}^{+}$signals is the opposite of what was reported above in terms of photoinduced effects. After one day, the four-line $\mathrm{Li}^{+}$signal can be seen without any exposure to laser light, and both signals decrease in intensity when the laser is turned on, meaning that the Fe ion is converted from its $3+$ state to a different charge state upon illumination. The behavior of the isolated interstitial $\mathrm{Li}^{+}$defect reported in the previous chapter is not any different one day after heating than it is one month later. This behavior suggests that the color of the crystal is a result of the trapping mechanisms of the $\mathrm{Fe}^{3+}$ ion adjacent an interstitial $\mathrm{Li}^{+}$ion. 


\subsection{Discussion}

It is highly doubtful that laser illumination causes the lithium ions themselves to move through the crystal at $5 \mathrm{~K}$; at such a low temperature, there is not enough thermal energy in the lattice to facilitate ionic conductivity. It is clear the laser creates electronhole pairs, with the electron and hole being trapped separately by defects in the crystal at low temperature. The photoinduced behavior of the signals in Figure 6.3 indicates that there are isolated $\mathrm{Fe}^{3+}$ defects present prior to laser illumination. The increase in the $\mathrm{Fe}^{3+}$ signal intensity indicates that the laser converts iron in either the $2+$ or $4+$ charge state into $\mathrm{Fe}^{3+}$ by trapping either an electron or hole. The iron defects that have $\mathrm{Lii}^{+}$ interstitial nearby exist in either the $2+$ or $4+$ charge state. Since the appearance of $\mathrm{Al}^{3+}$ hole centers directly accompanies the appearance of additional $\mathrm{Fe}^{3+}$ centers and the $\mathrm{Li}^{+}$ signal, I propose that the lithium-iron complex is a nonparamagnetic $\mathrm{Fe}^{4+}-\mathrm{Li}^{+}$complex prior to illumination. A similar defect was study by Jani et al. ${ }^{6}$ in silicon dioxide. In that material, an extra electron was trapped by interstitial $\mathrm{Li}^{+}$ions in an otherwise perfect lattice. This $\left[\mathrm{SiO}_{4} / \mathrm{Li}\right]^{0}$ defect was formed via a two-step irradiation process. First, ionizing radiation moves the $\mathrm{Li}^{+}$ion away from substitutional $\mathrm{Al}^{3+}$ defects when the sample temperature is at or above $200 \mathrm{~K}$. Since the $\left[\mathrm{SiO}_{4} / \mathrm{Li}\right]^{0}$ defect is only stable below $180 \mathrm{~K}$, the crystal was then immediately re-irradiated at $77 \mathrm{~K}$ to allow the $\mathrm{Li}^{+}$ions to diffuse to another defect site, adjacent to $\mathrm{Si}^{4+}$ ions.

In $\mathrm{TiO}_{2}$, no ionizing radiation is required to move the $\mathrm{Li}^{+}$ion next to a substitutional $\mathrm{Fe}^{4+}$ ion. Laser light causes electrons to form in the conduction band that are trapped at the lithium-iron complex, resulting in a paramagnetic $\mathrm{Fe}^{3+}-\mathrm{Li}^{+}$center. Valence-band holes are then trapped at substitutional $\mathrm{Al}^{3+}$ centers. Further evidence 
promoting this model is the fact that the $\mathrm{Al}^{3+}$ hole center signals do not appear in the asreceived crystal. If there is no $\mathrm{Fe}^{4+}-\mathrm{Li}^{+}$complex in the crystal to serve as an electron trap, there would be no compensation for an aluminum defect to trap a hole, leaving it nonparamagnetic and undetectable. Hence the $\mathrm{Fe}^{3+}-\mathrm{Li}^{+}$complex is an electron trap. The $\mathrm{Fe}^{3+}$ signal is part of a five-set $\mathrm{S}=5 / 2$ system that has a large zero field splitting. This results in a wide splitting of the EPR lines, placing the lowest-field line at a relatively high $g$ value.

One major question that exists with this defect is why the light is needed to observe the $\mathrm{Li}^{+}$signal in the undoped Crystec sample, but not the iron-doped sample. One possible answer has to do with the increased concentration of oxygen vacancies in iron-doped crystals. Carrettin et al. ${ }^{7}$ showed that increased iron concentration facilitates the formation of surface oxygen vacancies. Roldan et al. ${ }^{8}$ then provided theoretical evidence that the incorporation of $\mathrm{Fe}^{3+}$ in the rutile lattice leads to increased concentration of oxygen vacancies. EPR studies indicate (not reported here) that an increase in iron concentration does indeed result in more intense oxygen vacancy EPR signals. An increase in the number of oxygen vacancies increases the number of free electrons in the crystal (increases the Fermi level). Therefore, when cooling the crystal down to $5 \mathrm{~K}$, these free electrons "freeze" at available electron traps. This is similar to the phenomenon observed with regard to hydrogen donor centers in reduced and unreduced $\mathrm{TiO}_{2}$ (Chapter 3). The $\mathrm{Fe}^{3+}-\mathrm{Li}^{+}$complex then provides a stable electron trap without the need for laser light. 


\section{Chapter 6 References}

1. D. L. Carter and A Okaya, Physical Review 18, 1485 (1960).

2. S. S. Kim, S. S. Jun, and M. J. Park, J. of the Korean Phys. Soc. 23, 73 (1990).

3. S. Guler, B. Rameev, R. I. Khaibullin, H. Bayrakdar, and B. Aktas, Phys. Stat. Sol. 203,1533 (2006).

4. D. Zwingel, Solid State Commun. 20, 397 (1976).

5. Shan Yang, A. T. Brant, and L. E. Halliburton Phys. Rev. B 82, 035209 (2010).

6. M. G. Jani, L. E. Halliburton, and A. Halperinin, Phys. Rev. Lett. 56, 1392 (1986).

7. S. Carrettin, Y. Hao, V. Aguilar-Guerrero, B. C. Gates, S. Trasobares, J. J. Calvino, and A. Corma, Chem - Eur. J. 13, 7771 (2007).

8. A. Roldan, M. Boronat, A. Corma, and F. Illas, J. Phys. Chem. C 114, 6511 (2010). 


\section{Appendix A.1}

\section{Hydrogen in $\mathrm{TiO}_{2}$ g Fitting}

This program determines the "best" set of g-matrix parameters for the hydrogen donor in TiO2. This program can be modified for use in determining the principal values and principal axis directions for any EPR problem. Bold text is not code that contributes to the program.

Input data consists of $\mathbf{4 2}$ magnetic field values and their corresponding microwave frequencies. The output is $\mathbf{4}$ parameters (three principal values and one Euler angle).

clear all

format long

Planck $=6.626069$

Planck's constant

$\mathrm{B}=9.274009$ /Planck;

$\mathrm{CTR}=\mathrm{pi} / 180$;

Bohr magneton divided by Planck's constant

Conversion constant, degrees to radians

Initial values of the spin-Hamiltonian parameters:

Four for the g matrix (three principal values and one Euler angle).

$\mathrm{P}(1)=1.976$

$\mathrm{P}(2)=1.972$

$\mathrm{P}(3)=1.9405$

$\mathrm{P}(4)=19^{*} \mathrm{CTR}$;

$\mathrm{P}(5)=0 * \mathrm{CTR}$

$\mathrm{P}(6)=0 * \mathrm{CTR}$

Step sizes for the parameters:

$\mathrm{gg}=0.00001 ; \quad$ step size for the principal values

delta $=0.001 ; \quad$ step size for the angle

$\operatorname{step}(1)=\mathrm{gg}$;

$\operatorname{step}(2)=g g$;

$\operatorname{step}(3)=$ gg;

step $(4)=$ delta;

$\operatorname{sum} 2=0 ;$

sum1 = hydrogen_g_fitting_sub $(\mathrm{P}, \mathrm{B})$;

while sum $2<$ sum 1 


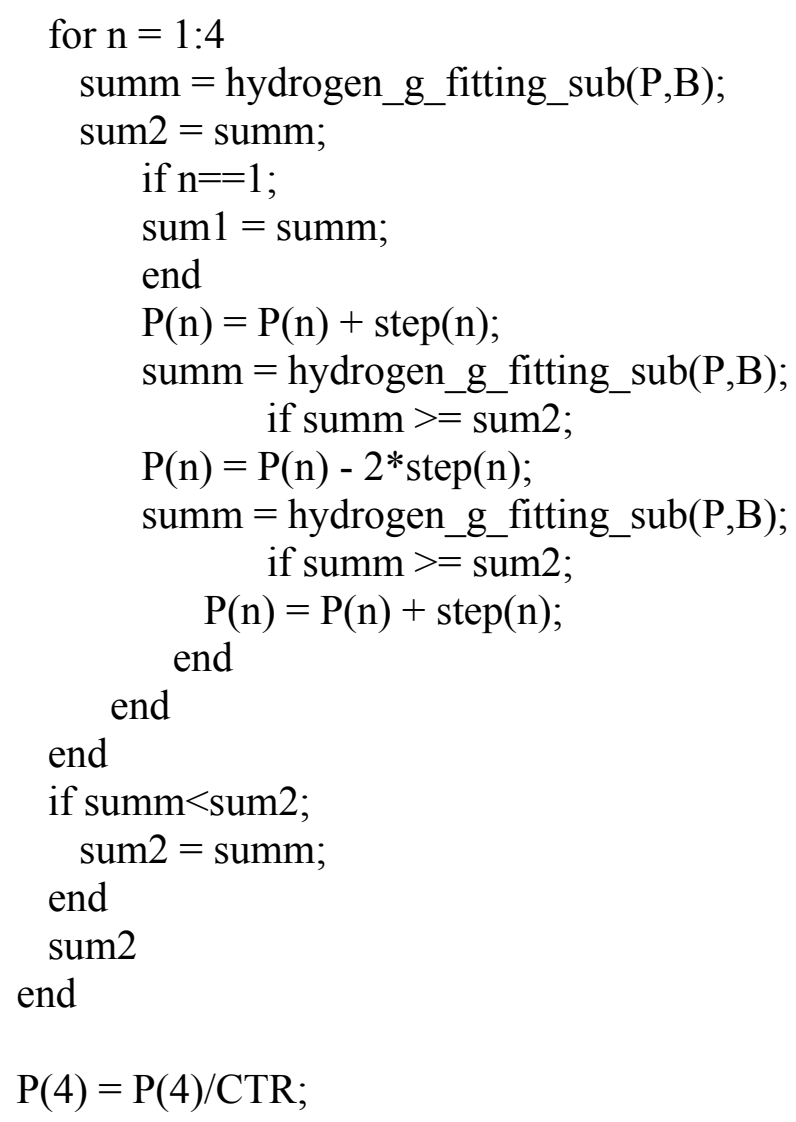

End of program.

\section{Hydrogen in $\mathrm{TiO}_{2} \mathrm{~g}$ fitting subroutine}

This subroutine is used in conjunction with the above g-fitting program to determine the best set of g-matrix parameters for the hydrogen donor in $\mathrm{TiO}_{2}$.

It calculates a sum of the frequency differences squared and returns the value to the main program. The input data are the measured magnetic fields and microwave frequencies.

function summ $=$ hydrogen_g_fitting_sub(P,B)

$\mathrm{CTR}=\mathrm{pi} / 180$

$\mathrm{G}$ is the $3 \times 3$ rotation matrix which takes the principal axes of the $\mathrm{g}$ matrix into the crystal coordinate system. 
$\mathrm{G}(1,1)=\cos (\mathrm{P}(4))$

$\mathrm{G}(1,2)=\sin (\mathrm{P}(4))$;

$\mathrm{G}(1,3)=0$;

$\mathrm{G}(2,1)=-\sin (\mathrm{P}(4))$;

$\mathrm{G}(2,2)=\cos (\mathrm{P}(4))$;

$\mathrm{G}(2,3)=0$;

$\mathrm{G}(3,1)=0$;

$\mathrm{G}(3,2)=0$

$\mathrm{G}(3,3)=1$;

This is the raw, experimental data. It consists of 42 resonance field values, the corresponding microwave frequency, in $\mathrm{MHz}$, defect site $(\mathrm{K})$ and the orientation of the magnetic field. Alpha is the angle between the magnetic field and the c-axis while Beta is the angle between the [110] axis and the field.

$\mathrm{h}(1)=3479.54 ; \mathrm{FRQ}(1)=9449.854 ; \mathrm{K}(1)=1 ; \operatorname{Alpha}(1)=0 * \mathrm{CTR} ; \operatorname{Beta}(1)=0 * \mathrm{CTR}$;

$\mathrm{h}(2)=3476.07 ; \mathrm{FRQ}(2)=9450.052 ; \mathrm{K}(2)=3 ; \operatorname{Alpha}(2)=15 * \mathrm{CTR} ; \operatorname{Beta}(2)=0 * \mathrm{CTR}$; $\mathrm{h}(3)=3476.07 ; \mathrm{FRQ}(3)=9450.052 ; \mathrm{K}(3)=1 ; \operatorname{Alpha}(3)=15 * \mathrm{CTR} ; \operatorname{Beta}(3)=0 * \mathrm{CTR}$;

$\mathrm{h}(4)=3463.02 ; \mathrm{FRQ}(4)=9450.444 ; \mathrm{K}(4)=3 ; \operatorname{Alpha}(4)=30 * \mathrm{CTR} ; \operatorname{Beta}(4)=0 * \mathrm{CTR}$; $\mathrm{h}(5)=3464.23 ; \mathrm{FRQ}(5)=9450.444 ; \mathrm{K}(5)=1 ; \operatorname{Alpha}(5)=30 * \mathrm{CTR} ; \operatorname{Beta}(5)=0 * \mathrm{CTR}$;

$\mathrm{h}(6)=3453.28 ; \mathrm{FRQ}(6)=9450.884 ; \mathrm{K}(6)=3 ; \mathrm{Alpha}(6)=40 * \mathrm{CTR} ; \operatorname{Beta}(6)=0 * \mathrm{CTR}$; $\mathrm{h}(7)=3455.29 ; \mathrm{FRQ}(7)=9450.884 ; \mathrm{K}(7)=1 ; \operatorname{Alpha}(7)=40 * \mathrm{CTR} ; \operatorname{Beta}(7)=0 * \mathrm{CTR}$;

$\mathrm{h}(8)=3443.14 ; \mathrm{FRQ}(8)=9451.311 ; \mathrm{K}(8)=3 ; \operatorname{Alpha}(8)=50 * \mathrm{CTR} ; \mathrm{Beta}(8)=0 * \mathrm{CTR}$; $\mathrm{h}(9)=3445.76 ; \mathrm{FRQ}(9)=9451.311 ; \mathrm{K}(9)=1 ; \operatorname{Alpha}(9)=50 * \mathrm{CTR} ; \operatorname{Beta}(9)=0 * \mathrm{CTR}$;

$\mathrm{h}(10)=3431.99 ; \mathrm{FRQ}(10)=9451.703 ; \mathrm{K}(10)=3 ; \operatorname{Alpha}(10)=60 * \mathrm{CTR} ; \operatorname{Beta}(10)=0 * \mathrm{CTR}$; $\mathrm{h}(11)=3435.61 ; \mathrm{FRQ}(11)=9451.703 ; \mathrm{K}(11)=1 ; \operatorname{Alpha}(11)=60 * \mathrm{CTR} ; \operatorname{Beta}(11)=0 * \mathrm{CTR}$;

$\mathrm{h}(12)=3424.76 ; \mathrm{FRQ}(12)=9452.064 ; \mathrm{K}(12)=3 ; \mathrm{Alpha}(12)=70 * \mathrm{CTR} ; \operatorname{Beta}(12)=0 * \mathrm{CTR}$; $\mathrm{h}(13)=3428.59 ; \mathrm{FRQ}(13)=9452.064 ; \mathrm{K}(13)=1 ; \mathrm{Alpha}(13)=70 * \mathrm{CTR} ; \operatorname{Beta}(13)=0 * \mathrm{CTR}$;

$\mathrm{h}(14)=3419.32 ; \mathrm{FRQ}(14)=9452.145 ; \mathrm{K}(14)=3 ; \operatorname{Alpha}(14)=80 * \mathrm{CTR} ; \operatorname{Beta}(14)=0 * \mathrm{CTR}$; $\mathrm{h}(15)=3422.86 ; \mathrm{FRQ}(15)=9452.145 ; \mathrm{K}(15)=1 ; \operatorname{Alpha}(15)=80 * \mathrm{CTR} ; \operatorname{Beta}(15)=0 * \mathrm{CTR}$;

$\mathrm{h}(16)=3417.19 ; \mathrm{FRQ}(16)=9452.300 ; \mathrm{K}(16)=3 ; \operatorname{Alpha}(16)=90 * \mathrm{CTR} ; \operatorname{Beta}(16)=0 * \mathrm{CTR}$; $\mathrm{h}(17)=3421.85 ; \mathrm{FRQ}(17)=9452.300 ; \mathrm{K}(17)=1 ; \mathrm{Alpha}(17)=90 * \mathrm{CTR} ; \operatorname{Beta}(17)=0 * \mathrm{CTR}$;

\section{Data from rotating from [001] to [100]}

$\mathrm{h}(18)=3477.41 ; \mathrm{FRQ}(18)=9444.230 ; \mathrm{K}(18)=1 ; \operatorname{Alpha}(18)=0 * \mathrm{CTR} ; \operatorname{Beta}(18)=45 * \mathrm{CTR}$;

$h(19)=3423.71 ; \mathrm{FRQ}(19)=9468.345 ; \mathrm{K}(19)=2 ; \operatorname{Alpha}(19)=90^{*} \mathrm{CTR} ; \operatorname{Beta}(19)=45^{*} \mathrm{CTR}$; 
$\mathrm{h}(20)=3427.29 ; \mathrm{FRQ}(20)=9468.345 ; \mathrm{K}(20)=1 ; \operatorname{Alpha}(20)=90 * \mathrm{CTR} ; \operatorname{Beta}(20)=45 * \mathrm{CTR}$;

\section{Data from rotating from [110] to [110]}

$\mathrm{h}(21)=3422.90 ; \mathrm{FRQ}(21)=9467.857 ; \mathrm{K}(21)=3 ; \operatorname{Alpha}(21)=90 * \mathrm{CTR} ; \operatorname{Beta}(21)=0 * \mathrm{CTR}$; $\mathrm{h}(22)=3427.44 ; \mathrm{FRQ}(22)=9467.857 ; \mathrm{K}(22)=1 ; \mathrm{Alpha}(22)=90 * \mathrm{CTR} ; \operatorname{Beta}(22)=0 * \mathrm{CTR}$;

$\mathrm{h}(23)=3422.34 ; \mathrm{FRQ}(23)=9467.895 ; \mathrm{K}(23)=4 ; \operatorname{Alpha}(23)=90 * \mathrm{CTR} ; \operatorname{Beta}(23)=15 * \mathrm{CTR}$; $\mathrm{h}(24)=3424.03 ; \mathrm{FRQ}(24)=9467.895 ; \mathrm{K}(24)=3 ; \mathrm{Alpha}(24)=90 * \mathrm{CTR} ; \operatorname{Beta}(24)=15^{*} \mathrm{CTR}$; $\mathrm{h}(25)=3426.25 ; \mathrm{FRQ}(25)=9467.895 ; \mathrm{K}(25)=2 ; \operatorname{Alpha}(25)=90 * \mathrm{CTR} ; \operatorname{Beta}(25)=15 * \mathrm{CTR}$; $\mathrm{h}(26)=3428.07 ; \mathrm{FRQ}(26)=9467.895 ; \mathrm{K}(26)=1 ; \operatorname{Alpha}(26)=90 * \mathrm{CTR} ; \operatorname{Beta}(26)=15 * \mathrm{CTR}$;

$\mathrm{h}(27)=3422.68 ; \mathrm{FRQ}(27)=9468.125 ; \mathrm{K}(27)=4 ; \operatorname{Alpha}(27)=90 * \mathrm{CTR} ; \operatorname{Beta}(27)=30 * \mathrm{CTR}$; $\mathrm{h}(28)=3424.99 ; \mathrm{FRQ}(28)=9468.125 ; \mathrm{K}(28)=2 ; \operatorname{Alpha}(28)=90 * \mathrm{CTR} ; \operatorname{Beta}(28)=30 * \mathrm{CTR}$; $\mathrm{h}(29)=3425.59 ; \mathrm{FRQ}(29)=9468.125 ; \mathrm{K}(29)=3 ; \operatorname{Alpha}(29)=90 * \mathrm{CTR} ; \operatorname{Beta}(29)=30 * \mathrm{CTR}$; $\mathrm{h}(30)=3427.98 ; \mathrm{FRQ}(30)=9468.125 ; \mathrm{K}(30)=1 ; \operatorname{Alpha}(30)=90 * \mathrm{CTR} ; \operatorname{Beta}(30)=30 * \mathrm{CTR}$;

$\mathrm{h}(31)=3423.71 ; \mathrm{FRQ}(31)=9450.884 ; \mathrm{K}(31)=2 ; \operatorname{Alpha}(31)=90 * \mathrm{CTR} ; \operatorname{Beta}(31)=45 * \mathrm{CTR}$; $\mathrm{h}(32)=3427.29 ; \mathrm{FRQ}(32)=9450.884 ; \mathrm{K}(32)=1 ; \operatorname{Alpha}(32)=90 * \mathrm{CTR} ; \operatorname{Beta}(32)=45^{*} \mathrm{CTR}$;

$\mathrm{h}(33)=3422.81 ; \mathrm{FRQ}(33)=9468.539 ; \mathrm{K}(33)=2 ; \mathrm{Alpha}(33)=90 * \mathrm{CTR} ; \operatorname{Beta}(33)=60 * \mathrm{CTR}$; $\mathrm{h}(34)=3425.14 ; \mathrm{FRQ}(34)=9468.539 ; \mathrm{K}(34)=4 ; \mathrm{Alpha}(34)=90 * \mathrm{CTR} ; \operatorname{Beta}(34)=60 * \mathrm{CTR}$; $\mathrm{h}(35)=3425.74 ; \mathrm{FRQ}(35)=9468.539 ; \mathrm{K}(35)=1 ; \operatorname{Alpha}(35)=90 * \mathrm{CTR} ; \operatorname{Beta}(35)=60 * \mathrm{CTR}$; $\mathrm{h}(36)=3428.15 ; \mathrm{FRQ}(36)=9468.539 ; \mathrm{K}(36)=3 ; \operatorname{Alpha}(36)=90 * \mathrm{CTR} ; \operatorname{Beta}(36)=60 * \mathrm{CTR}$;

$\mathrm{h}(37)=3422.67 ; \mathrm{FRQ}(37)=9468.816 ; \mathrm{K}(37)=2 ; \operatorname{Alpha}(37)=90 * \mathrm{CTR} ; \operatorname{Beta}(37)=75 * \mathrm{CTR}$; $\mathrm{h}(38)=3424.42 ; \mathrm{FRQ}(38)=9468.816 ; \mathrm{K}(38)=1 ; \operatorname{Alpha}(38)=90 * \mathrm{CTR} ; \operatorname{Beta}(38)=75 * \mathrm{CTR}$; $\mathrm{h}(39)=3426.58 ; \mathrm{FRQ}(39)=9468.816 ; \mathrm{K}(39)=4 ; \operatorname{Alpha}(39)=90 * \mathrm{CTR} ; \operatorname{Beta}(39)=75^{*} \mathrm{CTR}$; $\mathrm{h}(40)=3428.49 ; \mathrm{FRQ}(40)=9468.816 ; \mathrm{K}(40)=3 ; \mathrm{Alpha}(40)=90 * \mathrm{CTR} ; \operatorname{Beta}(40)=75 * \mathrm{CTR}$;

$\mathrm{h}(41)=3423.18 ; \mathrm{FRQ}(41)=9468.603 ; \mathrm{K}(41)=1 ; \operatorname{Alpha}(41)=90 * \mathrm{CTR} ; \operatorname{Beta}(41)=90 * \mathrm{CTR}$; $\mathrm{h}(42)=3427.83 ; \mathrm{FRQ}(42)=9468.603 ; \mathrm{K}(42)=3 ; \operatorname{Alpha}(42)=90 * \mathrm{CTR} ; \operatorname{Beta}(42)=90 * \mathrm{CTR}$;

datapoints $=$ length $(\mathrm{h})$

for $\mathrm{nn}=1$ :datapoints

$\mathrm{HH}=\mathrm{h}(\mathrm{nn})$;

$\mathrm{k}=\mathrm{K}(\mathrm{nn})$;

alpha $=\operatorname{Alpha}(\mathrm{nn})$;

beta $=\operatorname{Beta}(n n)$; 
$\mathrm{RM}$ is the 3x3 rotation matrix which takes the crystal coordinate system into the magnetic field coordinate system.

$\operatorname{RM}(1,1)=\cos ($ alpha $) * \cos ($ beta $)$;

$\operatorname{RM}(1,2)=-\sin ($ beta $)$;

$\operatorname{RM}(1,3)=\sin ($ alpha $) * \cos ($ beta $)$;

$\operatorname{RM}(2,1)=\cos ($ alpha $) * \sin ($ beta $)$;

$\operatorname{RM}(2,2)=\cos ($ beta $)$;

$\operatorname{RM}(2,3)=\sin ($ alpha $) * \sin ($ beta $)$

$\operatorname{RM}(3,1)=-\sin ($ alpha $)$;

$\operatorname{RM}(3,2)=0$;

$\operatorname{RM}(3,3)=\cos ($ alpha $)$;

The matrix $R$ represents the four magnetically inequivalent sites of the hydrogen defect. Each matrix takes the coordinate system of sites 2 through 4 and rotates it back to site 1.

if $\mathrm{k}==1$

$$
\begin{aligned}
& \mathrm{R}(1,1)=1 ; \mathrm{R}(1,2)=0 ; \mathrm{R}(1,3)=0 ; \\
& \mathrm{R}(2,1)=0 ; \mathrm{R}(2,2)=1 ; \mathrm{R}(2,3)=0 ; \\
& \mathrm{R}(3,1)=0 ; \mathrm{R}(3,2)=0 ; \mathrm{R}(3,3)=1 ; \\
& \mathrm{RT}=\mathrm{R} * \mathrm{RM} ;
\end{aligned}
$$

elseif $\mathrm{k}==2$

$$
\begin{aligned}
& \mathrm{R}(1,1)=1 ; \mathrm{R}(1,2)=0 ; \mathrm{R}(1,3)=0 ; \\
& \mathrm{R}(2,1)=0 ; \mathrm{R}(2,2)=-1 ; \mathrm{R}(2,3)=0 ; \\
& \mathrm{R}(3,1)=0 ; \mathrm{R}(3,2)=0 ; \mathrm{R}(3,3)=-1 ; \\
& \mathrm{RT}=\mathrm{R} * \mathrm{RM} ; \\
& \text { elseif } \mathrm{k}==3 \\
& \mathrm{R}(1,1)=0 ; \mathrm{R}(1,2)=1 ; \mathrm{R}(1,3)=0 ; \\
& \mathrm{R}(2,1)=1 ; \mathrm{R}(2,2)=0 ; \mathrm{R}(2,3)=0 ; \\
& \mathrm{R}(3,1)=0 ; \mathrm{R}(3,2)=0 ; \mathrm{R}(3,3)=-1 ; \\
& \mathrm{RT}=\mathrm{R} * \mathrm{RM} ; \\
& \text { elseif } \mathrm{k}==4 \\
& \mathrm{R}(1,1)=0 ; \mathrm{R}(1,2)=1 ; \mathrm{R}(1,3)=0 ; \\
& \mathrm{R}(2,1)=-1 ; \mathrm{R}(2,2)=0 ; \mathrm{R}(2,3)=0
\end{aligned}
$$




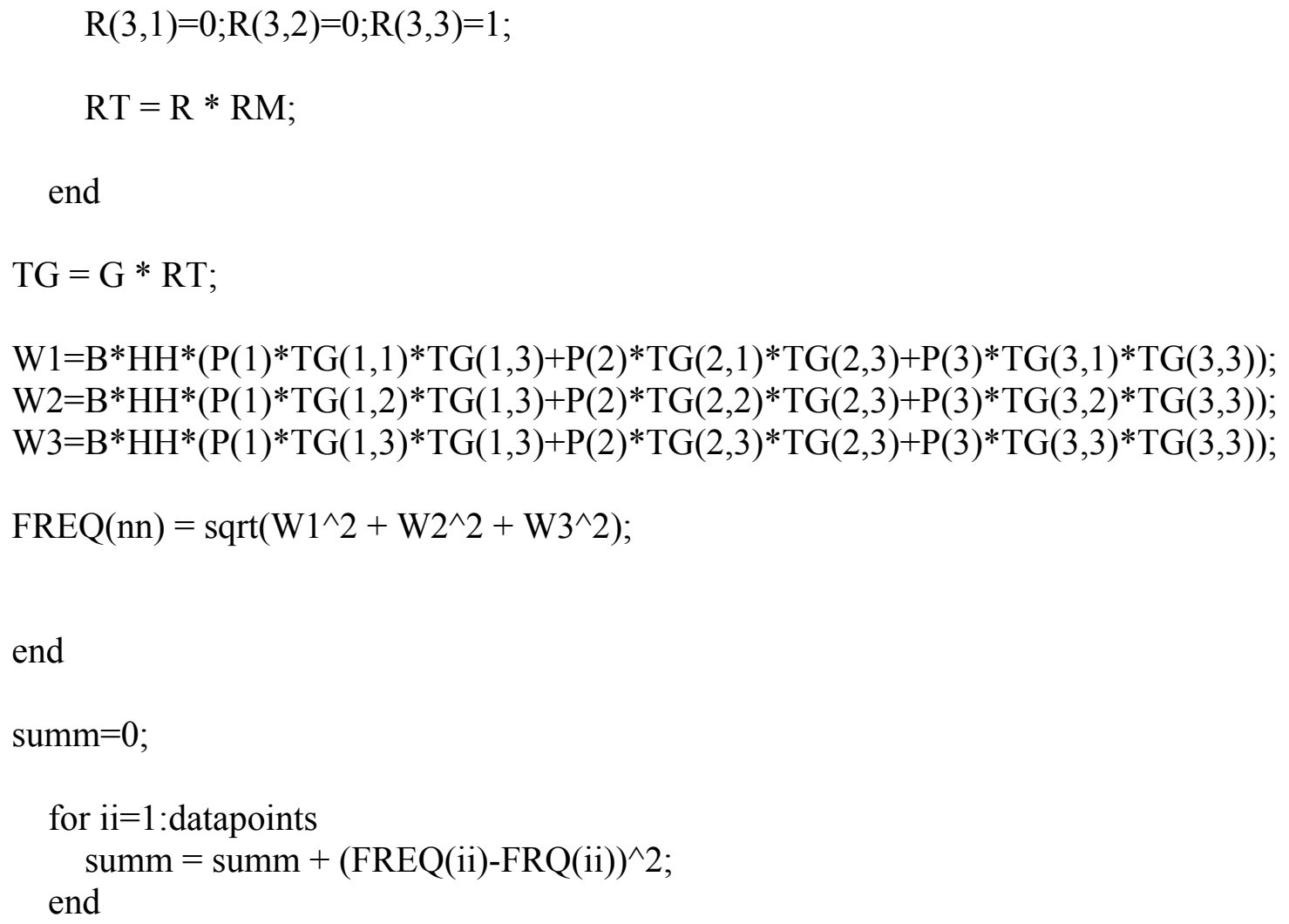

End of subroutine 


\section{Appendix A.2}

\section{Hydrogen in $\mathrm{TiO}_{2}$ A Fitting}

This program determines the "best" set of principal values and principal axis directions for the hyperfine matrix of the neutral hydrogen donor in $\mathrm{TiO}_{2}$

Input data consists of 10 ENDOR frequencies and their corresponding magnetic field values. The output is 4 parameters (three principal values and one Euler angle). Bold text is not code that contributes to the program.

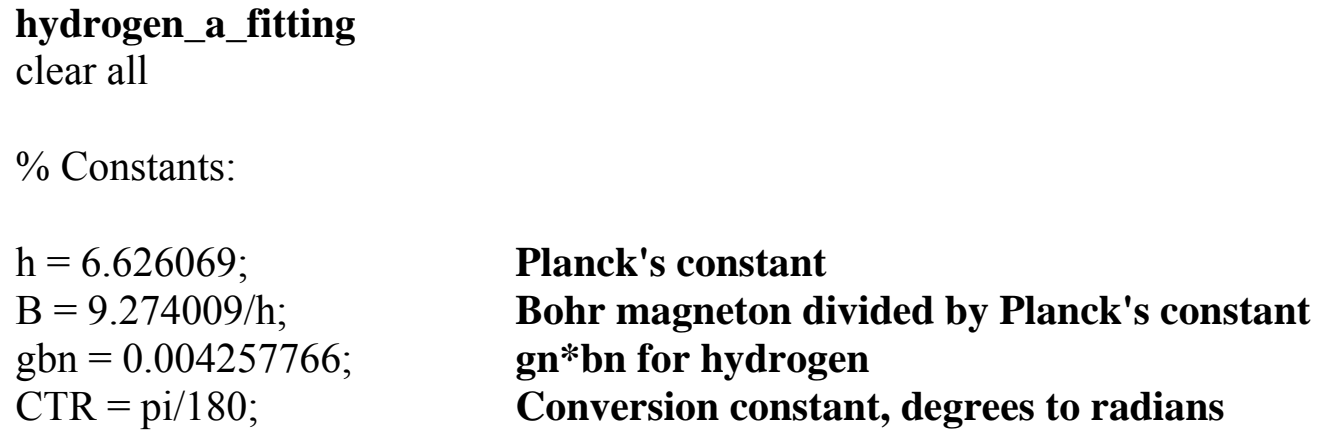

Initial values of the spin-Hamiltonian parameters:

-PP - Four for the g matrix (three principal values and one angle). These parameters were determined with another fitting program and are held constant here.

- P - Four for the A matrix (three principal values and one angle). These are varied to find the best fit.

$\operatorname{PP}(1)=1.9732$

$\mathrm{PP}(2)=1.9765$

$\operatorname{PP}(3)=1.9405$

$\mathrm{PP}(4)=19.0 * \mathrm{CTR}$

$\mathrm{P}(1)=0.4$

$\mathrm{P}(2)=0.4$

$\mathrm{P}(3)=0.4$

$\mathrm{P}(4)=22 * \mathrm{CTR}$

Step sizes for the parameters:

aa $=0.001 ; \quad$ step size for the principal values

delta $=0.01 * \mathrm{CTR} ; \quad$ step size for the angle

$\operatorname{step}(1)=$ aa;

$\operatorname{step}(2)=$ aa;

$\operatorname{step}(3)=a a$; 


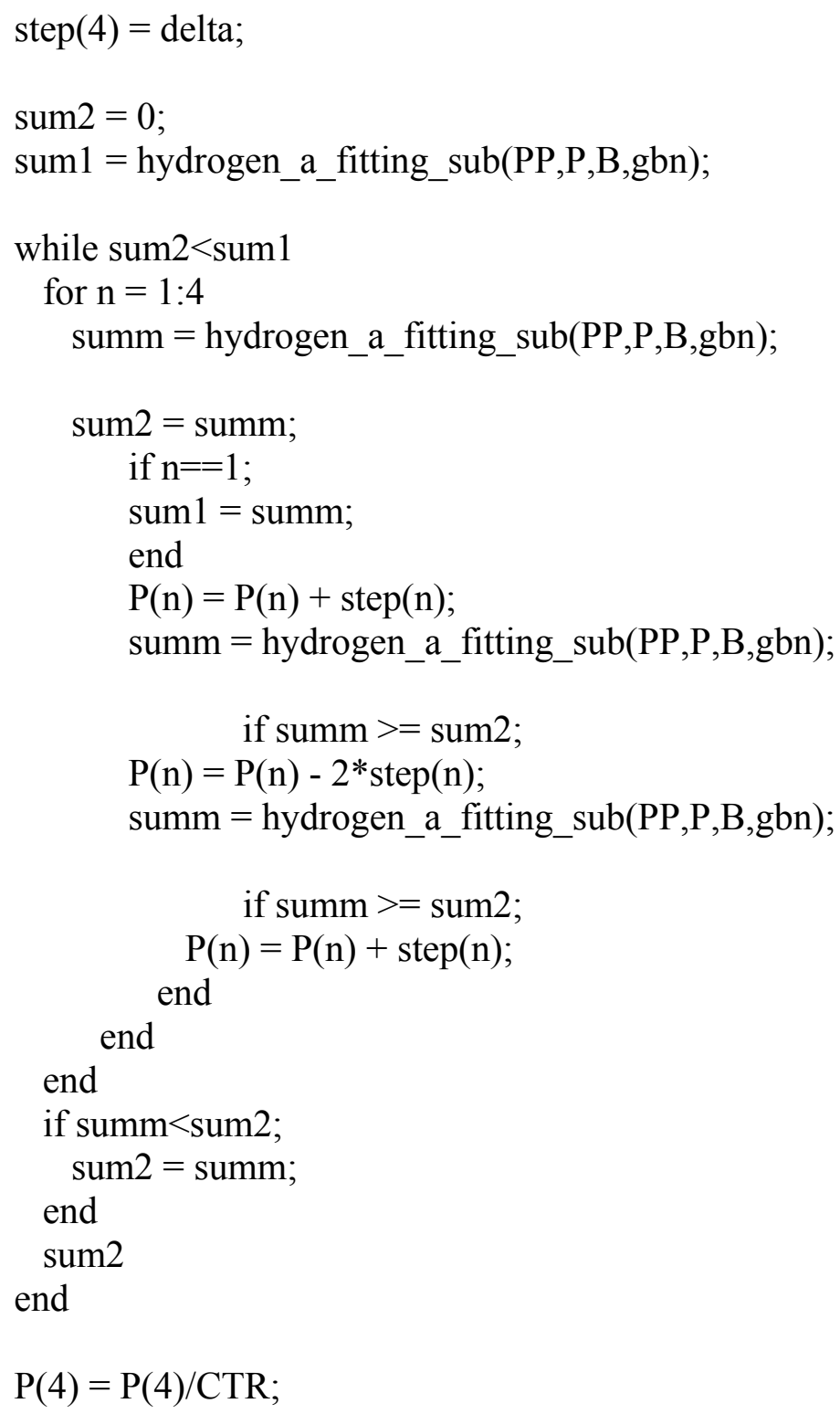

End of program.

\section{Hydrogen in $\mathrm{TiO}_{2}$ A fitting subroutine}

This subroutine is used in conjunction with the above A-fitting program to determine the best set of A-matrix parameters for the hydrogen donor in $\mathrm{TiO}_{2}$.

It calculates a sum of the frequency differences squared and returns the value to the main program. The input data are the measured magnetic fields and ENDOR 
frequencies. This program could be modified to solve the hyperfine matrix for any EPR/ENDOR problem.

function summ = SUMM_hydrogen_ENDOR_fitting $(\mathrm{PP}, \mathrm{P}, \mathrm{B}, \mathrm{gbn})$

$\mathrm{CTR}=\mathrm{pi} / 180$

$\mathrm{G}$ is the $3 \times 3$ rotation matrix which takes the principal axes of the $\mathrm{g}$ matrix into the crystal coordinate system.

$\mathrm{H}$ is the $3 \times 3$ rotation matrix which takes the principal axes of the A matrix into the crystal coordinate system.

$R$ is the $3 \times 3$ rotation matrix which takes the coordinate system for defect sites 2 through 8 back to the coordinate system for defect site 1.

$R M$ is the $3 \times 3$ rotation matrix which takes the crystal coordinate system into the magnetic field coordinate system.

$\mathrm{G}(1,1)=\cos (\mathrm{PP}(4))$;

$\mathrm{G}(1,2)=\sin (\mathrm{PP}(4))$;

$\mathrm{G}(1,3)=0$;

$\mathrm{G}(2,1)=-\sin (\mathrm{PP}(4))$;

$\mathrm{G}(2,2)=\cos (\mathrm{PP}(4))$;

$\mathrm{G}(2,3)=0$;

$\mathrm{G}(3,1)=0$;

$\mathrm{G}(3,2)=0$;

$\mathrm{G}(3,3)=1$;

$\mathrm{H}(1,1)=\cos (\mathrm{P}(4))$;

$\mathrm{H}(1,2)=\sin (\mathrm{P}(4))$;

$\mathrm{H}(1,3)=0$;

$\mathrm{H}(2,1)=-\sin (\mathrm{P}(4))$;

$\mathrm{H}(2,2)=\cos (\mathrm{P}(4))$;

$\mathrm{H}(2,3)=0$;

$\mathrm{H}(3,1)=0$;

$\mathrm{H}(3,2)=0$;

$\mathrm{H}(3,3)=1$;

This is the raw, experimental data. It consists of 10 resonance field values, the corresponding ENDOR frequency, in MHz, defect site (K), the line number (Line) and the orientation of the magnetic field. Alpha is the angle between the magnetic field and the c-axis while Beta is the angle between the [110] axis and the field. 


\section{Data along [001].}

$\mathrm{h}(1)=3490.88 ; \mathrm{FRQ}(1)=14.708 ; \mathrm{K}(1)=1 ; \operatorname{Line}(1)=1 ; \mathrm{Alpha}(1)=0 * \mathrm{CTR} ; \operatorname{Beta}(1)=0 * \mathrm{CTR}$;

$\mathrm{h}(2)=3490.88 ; \mathrm{FRQ}(2)=15.046 ; \mathrm{K}(2)=1 ; \operatorname{Line}(2)=2 ; \mathrm{Alpha}(2)=0 * \mathrm{CTR} ; \operatorname{Beta}(2)=0 * \mathrm{CTR}$;

\section{Data along [100].}

$\mathrm{h}(3)=3399.97 ; \mathrm{FRQ}(3)=14.347 ; \mathrm{K}(3)=1 ; \operatorname{Line}(3)=2 ; \mathrm{Alpha}(3)=90 * \mathrm{CTR} ; \operatorname{Beta}(3)=45 * \mathrm{CTR}$; $\mathrm{h}(4)=3399.97 ; \mathrm{FRQ}(4)=14.60 ; \mathrm{K}(4)=1 ; \operatorname{Line}(4)=1 ; \mathrm{Alpha}(4)=90 * \mathrm{CTR} ; \operatorname{Beta}(4)=45 * \mathrm{CTR}$; $\mathrm{h}(5)=3402.74 ; \mathrm{FRQ}(5)=14.253 ; \mathrm{K}(5)=2 ; \operatorname{Line}(5)=1 ; \mathrm{Alpha}(5)=90 * \mathrm{CTR} ; \operatorname{Beta}(5)=45^{*} \mathrm{CTR}$; $\mathrm{h}(6)=3402.74 ; \mathrm{FRQ}(6)=14.726 ; \mathrm{K}(6)=2 ; \operatorname{Line}(6)=2 ; \operatorname{Alpha}(6)=90 * \mathrm{CTR} ; \operatorname{Beta}(6)=45^{*} \mathrm{CTR}$;

\section{Data along [110].}

$\mathrm{h}(7)=3406.70 ; \mathrm{FRQ}(7)=14.281 ; \mathrm{K}(7)=3 ; \operatorname{Line}(7)=1 ; \mathrm{Alpha}(7)=90 * \mathrm{CTR} ; \operatorname{Beta}(7)=0 * \mathrm{CTR}$; $\mathrm{h}(8)=3406.70 ; \mathrm{FRQ}(8)=14.742 ; \mathrm{K}(8)=3 ; \operatorname{Line}(8)=2 ; \mathrm{Alpha}(8)=90 * \mathrm{CTR} ; \operatorname{Beta}(8)=0 * \mathrm{CTR}$; $\mathrm{h}(9)=3411.05 ; \mathrm{FRQ}(9)=14.407 ; \mathrm{K}(9)=1 ; \operatorname{Line}(9)=2 ; \mathrm{Alpha}(9)=90 * \mathrm{CTR} ; \operatorname{Beta}(9)=0 * \mathrm{CTR}$; $\mathrm{h}(10)=3411.05 ; \mathrm{FRQ}(10)=14.654 ; \mathrm{K}(10)=1 ; \operatorname{Line}(10)=1 ; \operatorname{Alpha}(10)=90 * \mathrm{CTR} ; \operatorname{Beta}(10)=0 *$ CTR;

datapoints $=$ length(h);

for $\mathrm{nn}=1$ :datapoints

$\mathrm{HH}=\mathrm{h}(\mathrm{nn})$;

$\mathrm{k}=\mathrm{K}(\mathrm{nn})$;

line $=$ Line $(\mathrm{nn})$;

alpha $=$ Alpha(nn);

beta $=\operatorname{Beta}(\mathrm{nn})$;

$\mathrm{RM}(1,1)=\cos (\mathrm{alpha}) * \cos ($ beta $)$;

$\operatorname{RM}(1,2)=-\sin ($ beta $)$;

$\operatorname{RM}(1,3)=\sin ($ alpha $) * \cos ($ beta $)$

$\mathrm{RM}(2,1)=\cos (\mathrm{alpha}) * \sin ($ beta);

$\operatorname{RM}(2,2)=\cos ($ beta $)$;

$\operatorname{RM}(2,3)=\sin ($ alpha $) * \sin ($ beta $)$;

$\operatorname{RM}(3,1)=-\sin ($ alpha);

$\operatorname{RM}(3,2)=0$;

$\operatorname{RM}(3,3)=\cos ($ alpha $)$;

if $\mathrm{k}==1$

SITE 1

$$
\begin{aligned}
& \mathrm{R}(1,1)=1 ; \mathrm{R}(1,2)=0 ; \mathrm{R}(1,3)=0 ; \\
& \mathrm{R}(2,1)=0 ; \mathrm{R}(2,2)=1 ; \mathrm{R}(2,3)=0 ; \\
& \mathrm{R}(3,1)=0 ; \mathrm{R}(3,2)=0 ; \mathrm{R}(3,3)=1 ;
\end{aligned}
$$


elseif $\mathrm{k}==2$

$\mathrm{R}(1,1)=1 ; \mathrm{R}(1,2)=0 ; \mathrm{R}(1,3)=0$;

$\mathrm{R}(2,1)=0 ; \mathrm{R}(2,2)=-1 ; \mathrm{R}(2,3)=0$;

$\mathrm{R}(3,1)=0 ; \mathrm{R}(3,2)=0 ; \mathrm{R}(3,3)=-1$;

elseif $\mathrm{k}==3$

$\mathrm{R}(1,1)=0 ; \mathrm{R}(1,2)=1 ; \mathrm{R}(1,3)=0$;

$\mathrm{R}(2,1)=1 ; \mathrm{R}(2,2)=0 ; \mathrm{R}(2,3)=0$;

$\mathrm{R}(3,1)=0 ; \mathrm{R}(3,2)=0 ; \mathrm{R}(3,3)=-1$;

elseif $\mathrm{k}==4$
SITE 2

SITE 3

\section{SITE 4}

$$
\begin{aligned}
& \mathrm{R}(1,1)=0 ; \mathrm{R}(1,2)=1 ; \mathrm{R}(1,3)=0 ; \\
& \mathrm{R}(2,1)=-1 ; \mathrm{R}(2,2)=0 ; \mathrm{R}(2,3)=0 \\
& \mathrm{R}(3,1)=0 ; \mathrm{R}(3,2)=0 ; \mathrm{R}(3,3)=1 ;
\end{aligned}
$$

end

$$
\begin{aligned}
& \mathrm{RT}=\mathrm{R} * \mathrm{RM} ; \\
& \mathrm{TG}=\mathrm{G} * \mathrm{RT} ; \\
& \mathrm{TH}=\mathrm{H} * \mathrm{RT} ;
\end{aligned}
$$

\section{The nine terms of the diagonalized Hamiltonian}

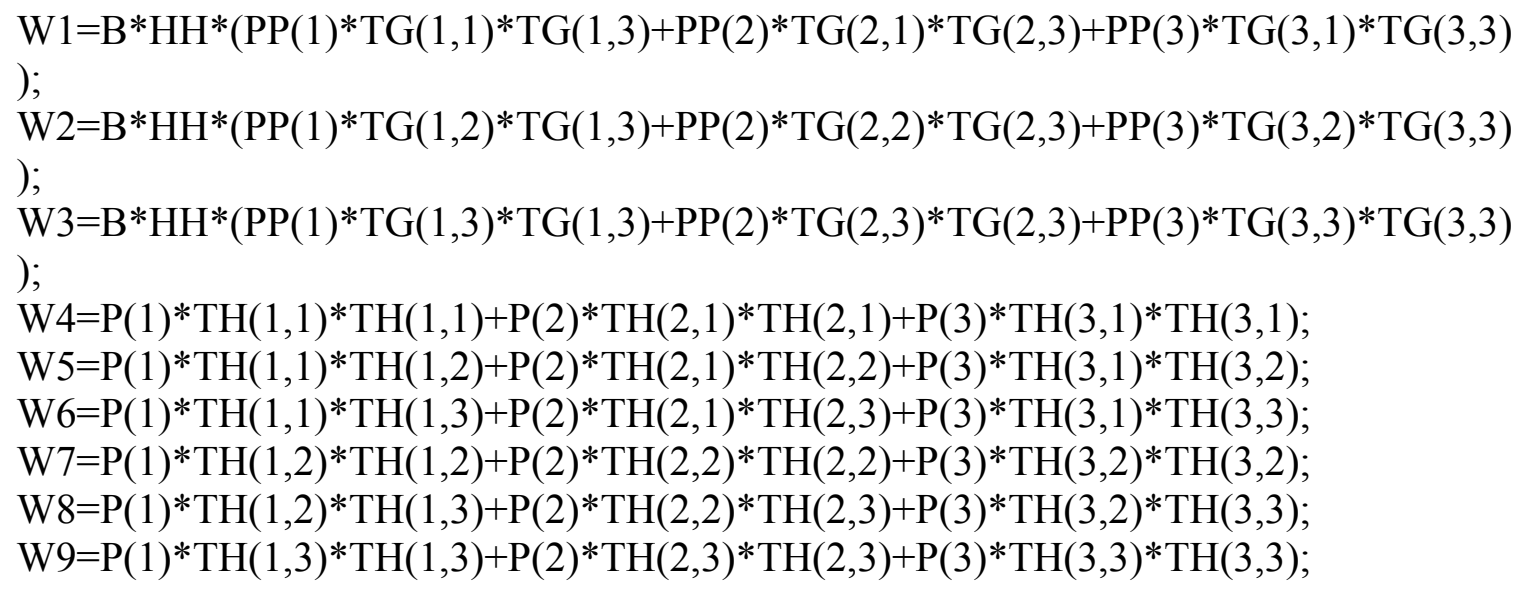

\section{Simplification of the above terms}

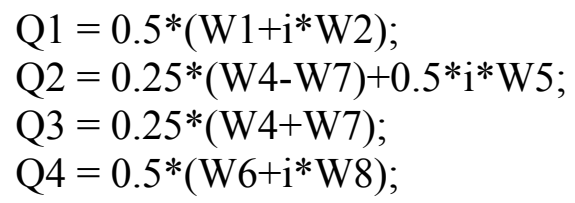


Ham is the matrix representing the spin-Hamiltonian:

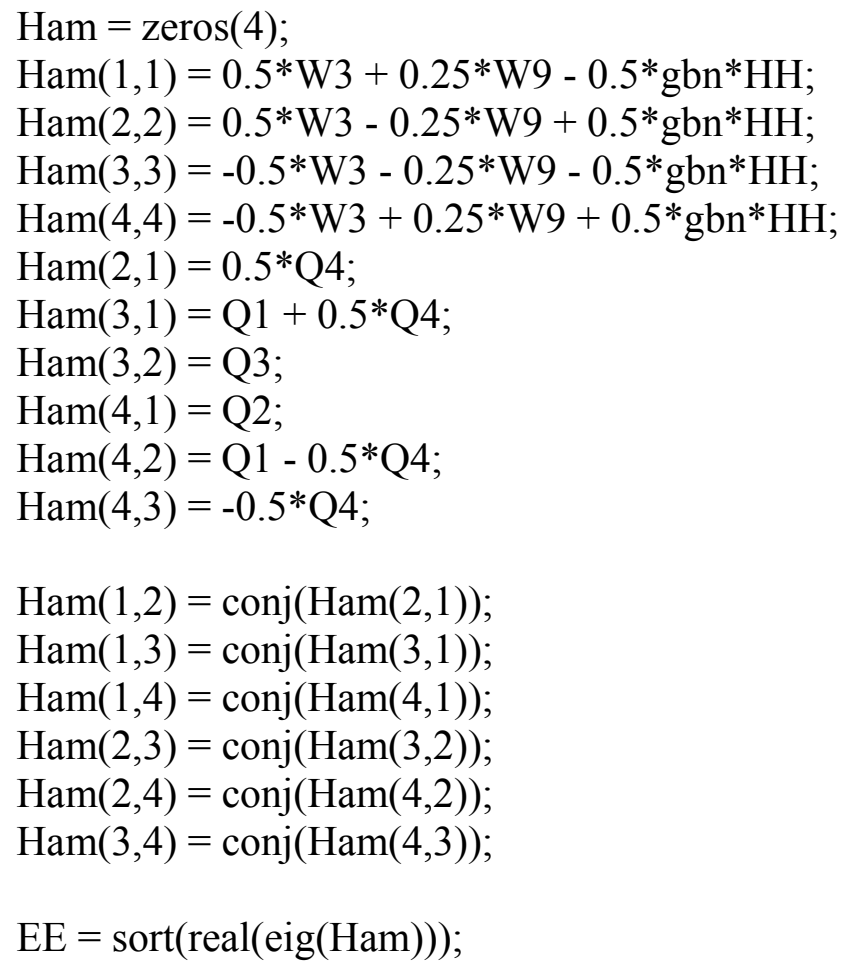

The differences in eigenvalues correspond to ENDOR transitions.

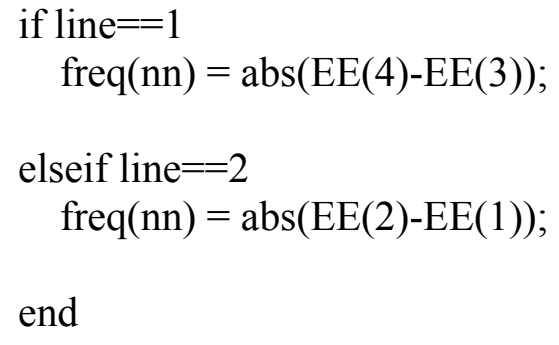

\section{End of Subroutine}




\section{Appendix B}

\section{Coordinate Transformations}

In order to determine the principal values and principal axis directions of the Hamiltonian parameters, one has be perform coordinate transformations in order to put the magnetic field axes and principal axis directions in the same reference frame as the crystal axes. Within this work, the magnetic field is oriented relative to the crystal axes by the angles $\alpha$ and $\beta$, as shown in Figure B.1.

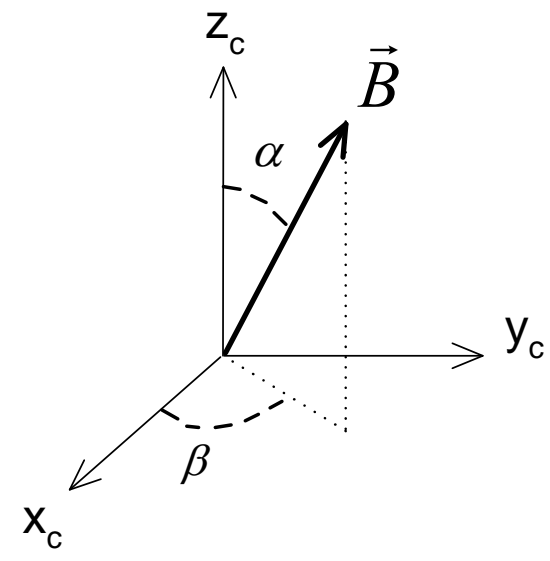

Figure B.1. Relationship between the magnetic field direction and the crystalline coordinate system.

The angle between the $\mathrm{z}$ axis of the crystal coordinate system and the field is denoted by $\alpha$, while $\beta$ is the angle between the crystalline $\mathrm{x}$ axis and the projection of $\vec{B}$ onto the $\mathrm{x}-\mathrm{y}$ plane. In its own frame, the field points along the $\mathrm{z}$ axis; i.e., $\vec{B}=B_{0} \hat{z}$. The following two matrices rotate the field vector from its own frame into the crystal frame ${ }^{1}$

$$
\left(\begin{array}{ccc}
\cos (\alpha) & 0 & \sin (\alpha) \\
0 & 1 & 0 \\
-\sin (\alpha) & 0 & \cos (\alpha)
\end{array}\right)
$$




$$
\left(\begin{array}{ccc}
\cos (\beta) & -\sin (\beta) & 0 \\
\sin (\beta) & \cos (\beta) & 0 \\
0 & 0 & 1
\end{array}\right) \longrightarrow \begin{aligned}
& \text { Clockwise rotation } \\
& \text { around } \mathrm{z}_{\mathrm{c}} \text { by } \beta
\end{aligned}
$$

Applying these two matrices consecutively to the vector $\vec{B}$ gives the rotation matrix for putting the magnetic field coordinate system into the the crystal coordinate system:

$$
\left(\begin{array}{ccc}
\cos (\alpha) \cos (\beta) & -\sin (\beta) & \sin (\alpha) \cos (\beta) \\
\cos (\alpha) \sin (\beta) & \cos (\beta) & \sin (\alpha) \sin (\beta) \\
-\sin (\alpha) & 0 & \cos (\alpha)
\end{array}\right)
$$

Similarly, the principal axes of the g matrix need to be rotated into the crystal frame.

Euler angles defined in Figure B.2 show how the g tensor axes relate to the crystal axes. The g-tensor frame is first rotated about its $\mathrm{z}$ axis by $\varphi$, then rotated about the new $\mathrm{x}$ axis by $\theta$, and finally rotated around the $z^{\prime}$ axis by $\psi$.

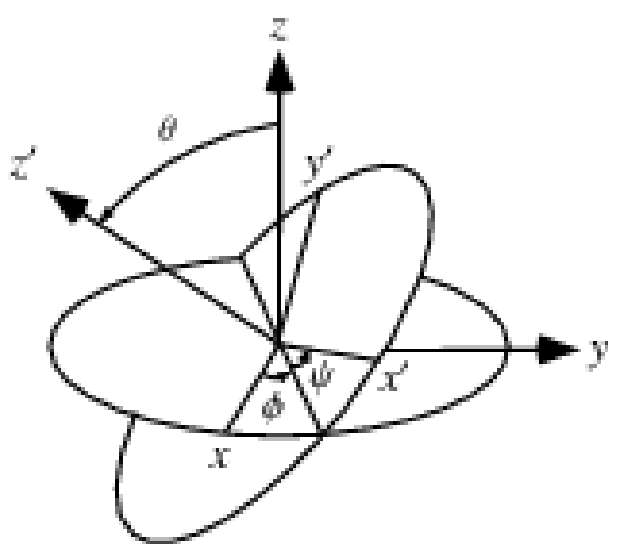

Figure B.2. Euler angles defined in the "zxz" convention. Picture was taken from Wolfram MathWorld (http://mathworld.wolfram.com/EulerAngles.html) 
The transformation matrix to go from the $\mathrm{g}$ frame to the crystal frame is:

$\left(\begin{array}{ccc}\cos (\psi) \cos (\phi)-\cos (\theta) \sin (\phi) \sin (\psi) & \cos (\phi) \sin (\psi) \cos (\theta)+\cos (\psi) \sin (\phi) & \sin (\psi) \sin (\theta) \\ -\sin (\psi) \cos (\phi)-\cos (\psi) \sin (\phi) \cos (\theta) & -\sin (\psi) \sin (\phi)+\cos (\phi) \cos (\theta) \cos (\psi) & \cos (\psi) \sin (\theta) \\ \sin (\phi) \sin (\theta) & -\sin (\theta) \cos (\phi) & \cos (\theta)\end{array}\right)$

This convention is also used when defining the Euler angles for the A and P matrices.

\section{Appendix B Reference}

1. H. Goldstein, C. P. Poole and J. L. Safko, Classical Mechanics, $3^{\text {rd }}$ ed. (Addison Wesley, 2002) 


\section{Appendix C}

\section{Experimental Data}

Table C.1: EPR angular dependence of neutral hydrogen donor. Field is rotated from the [001] to [110] directions

\begin{tabular}{|c|c|c|c|}
\hline Angle $\left({ }^{\circ}\right)$ & $\mathrm{B}_{\mathrm{r}}-1(\mathrm{G})$ & $\mathrm{B}_{\mathrm{r}}-2(\mathrm{G})$ & Frequency $(\mathrm{GHz})$ \\
\hline$[001]$ & 3479.67 & 3479.67 & 9.449854 \\
\hline 15 & 3476.25 & 3476.25 & 9.450052 \\
\hline 30 & 3463.15 & 3464.36 & 9.450444 \\
\hline 40 & 3453.27 & 3455.28 & 9.450884 \\
\hline 50 & 3443.18 & 3445.8 & 9.451311 \\
\hline 60 & 3432.09 & 3435.71 & 9.451703 \\
\hline 70 & 3424.85 & 3428.68 & 9.452064 \\
\hline 80 & 3419.6 & 3423.14 & 9.452145 \\
\hline$[110]$ & 3417.39 & 3422.05 & 9.452300 \\
\hline
\end{tabular}

Table C.2: EPR angular dependence of neutral hydrogen donor. Field is rotated from the [110] to [-110] directions

\begin{tabular}{|c|c|c|c|c|c|}
\hline Angle $\left(^{\circ}\right)$ & $\mathrm{Br}-1(\mathrm{G})$ & $\mathrm{Br}-2(\mathrm{G})$ & $\mathrm{Br}-3(\mathrm{G})$ & $\mathrm{Br}-4(\mathrm{G})$ & Frequency $(\mathrm{GHz})$ \\
\hline$[110]$ & 3423.05 & 3427.59 & 3423.05 & 3427.59 & 9.467857 \\
\hline 15 & 3422.46 & 3424.15 & 3426.37 & 3428.19 & 9.467895 \\
\hline 30 & 3422.74 & 3425.05 & 3425.65 & 3428.04 & 9.468125 \\
\hline$[100]$ & 3423.7 & 3427.28 & 3423.7 & 3427.28 & 9.468345 \\
\hline 60 & 3422.89 & 3425.22 & 3425.82 & 3428.23 & 9.468539 \\
\hline 75 & 3422.78 & 3424.53 & 3426.69 & 3428.6 & 9.468816 \\
\hline$[-110]$ & 3423.28 & 3427.93 & 3423.28 & 3427.93 & 9.468603 \\
\hline
\end{tabular}


Table C.3. ENDOR angular dependence of the neutral hydrogen donor in $\mathrm{TiO}_{2}$ from [001] to [100]

\begin{tabular}{|c|c|c|c|c|c|c|c|}
\hline Angle $\left(^{\circ}\right)$ & $\begin{array}{c}\text { EPR } \\
\text { Resonance } \\
\text { Magnetic } \\
\text { Field (G) }\end{array}$ & $\begin{array}{c}\text { EPR } \\
\text { Resonance } \\
\text { Magnetic } \\
\text { Field }(\mathrm{G})\end{array}$ & $\begin{array}{c}\text { ENDOR } \\
\text { Transition } \\
\text { Frequency } \\
(\mathrm{MHz})\end{array}$ & $\begin{array}{c}\text { ENDOR } \\
\text { Transition } \\
\text { Frequency } \\
(\mathrm{MHz})\end{array}$ & $\begin{array}{c}\text { ENDOR } \\
\text { Transition } \\
\text { Frequency } \\
(\mathrm{MHz})\end{array}$ & $\begin{array}{c}\text { ENDOR } \\
\text { Transition } \\
\text { Frequency } \\
(\mathrm{MHz})\end{array}$ & $\begin{array}{c}\text { Microwave } \\
\text { Frequency } \\
(\mathrm{GHz})\end{array}$ \\
\hline$[001]$ & 3491.61 & & 14.72 & 15.06 & & & 9.480087 \\
\hline 15 & 3486.21 & & 14.70 & 15.03 & & 9.478038 \\
\hline 30 & 3475.16 & & 14.65 & 15.00 & & 9.477554 \\
\hline 45 & 3457.13 & 3459.56 & 14.59 & 14.7 & 14.76 & 9.475996 \\
\hline 60 & 3441.24 & 3444.66 & 14.51 & 14.79 & & 9.474664 \\
\hline 75 & 3429.95 & 3433.98 & 14.4 & 14.51 & 14.75 & 14.84 & 9.473888 \\
\hline$[100]$ & 3426.51 & & 14.37 & 14.48 & 14.73 & 14.84 & 9.474193 \\
\hline
\end{tabular}

Table C.4. ENDOR angular dependence of the neutral hydrogen donor in $\mathrm{TiO}_{2}$ from [001] to [110]

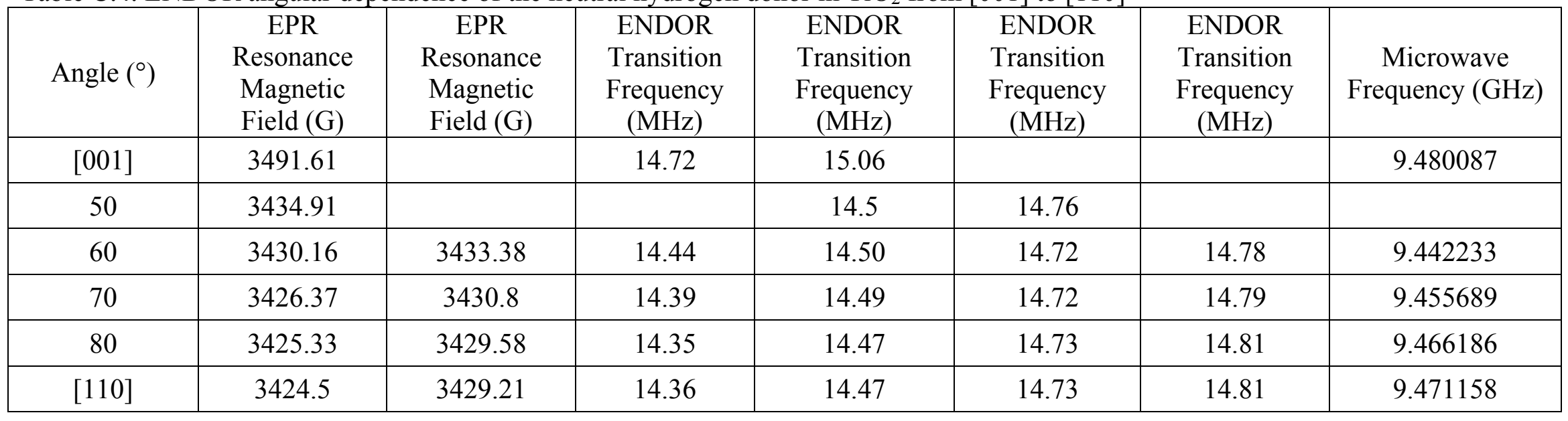


Table C.5. ENDOR angular dependence of the neutral hydrogen donor in $\mathrm{TiO}_{2}$ from [100] to [110]

\begin{tabular}{|c|c|c|c|c|c|}
\hline Angle $\left(^{\circ}\right)$ & $\begin{array}{c}\text { EPR Resonance } \\
\text { Magnetic Field (G) }\end{array}$ & $\begin{array}{c}\text { EPR Resonance } \\
\text { Magnetic Field (G) }\end{array}$ & $\begin{array}{c}\text { EPR Resonance } \\
\text { Magnetic Field (G) }\end{array}$ & $\begin{array}{c}\text { EPR Resonance } \\
\text { Magnetic Field (G) }\end{array}$ & $\begin{array}{c}\text { Microwave } \\
\text { Frequency }(\mathrm{GHz})\end{array}$ \\
\hline$[100]$ & 3399.84 & 3402.61 & & & 9.396914 \\
\hline 15 & 3406.75 & 3408.7 & 3410.02 & 3411.89 & 9.420223 \\
\hline 30 & 3410.09 & 3412 & 3413.66 & & 9.429728 \\
\hline$[110]$ & 3408.25 & 3412.61 & & & 9.422260 \\
\hline
\end{tabular}

Table C.5 Continued.

\begin{tabular}{|c|c|c|c|c|c|c|c|}
\hline Angle $\left(^{\circ}\right)$ & $\begin{array}{c}\text { ENDOR } \\
\text { Transition } \\
\text { Frequency } \\
(\mathrm{MHz})\end{array}$ & $\begin{array}{c}\text { ENDOR } \\
\text { Transition } \\
\text { Frequency } \\
(\mathrm{MHz})\end{array}$ & $\begin{array}{c}\text { ENDOR } \\
\text { Transition } \\
\text { Frequency } \\
(\mathrm{MHz})\end{array}$ & $\begin{array}{c}\text { ENDOR } \\
\text { Transition } \\
\text { Frequency } \\
(\mathrm{MHz})\end{array}$ & $\begin{array}{c}\text { ENDOR } \\
\text { Transition } \\
\text { Frequency } \\
(\mathrm{MHz})\end{array}$ & $\begin{array}{c}\text { ENDOR } \\
\text { Transition } \\
\text { Frequency } \\
(\mathrm{MHz})\end{array}$ & $\begin{array}{c}\text { Microwave } \\
\text { Frequency } \\
(\mathrm{GHz})\end{array}$ \\
\hline$[100]$ & 14.25 & 14.35 & 14.6 & 14.73 & & 14.7 & 14.84 \\
\hline 15 & 14.21 & 14.34 & 14.49 & 14.54 & 14.84 & 9.420223 \\
\hline 30 & 14.22 & 14.33 & 14.60 & 14.75 & & 9.429728 \\
\hline$[110]$ & 14.28 & 14.41 & 14.65 & 14.74 & & 9.422260 \\
\hline
\end{tabular}


Table C.6. EPR angular dependence of $\mathrm{Cu}^{2+}$ taken from [001] to [110]

\begin{tabular}{|c|c|c|c|c|c|c|c|c|c|}
\hline $\begin{array}{c}\text { Angle } \\
\left({ }^{\circ}\right)\end{array}$ & $\begin{array}{c}\mathrm{Br}-1 \\
(\mathrm{G})\end{array}$ & $\begin{array}{c}\mathrm{Br}-2 \\
(\mathrm{G})\end{array}$ & $\begin{array}{c}\mathrm{Br}-3 \\
(\mathrm{G})\end{array}$ & $\begin{array}{c}\mathrm{Br}-4 \\
(\mathrm{G})\end{array}$ & $\begin{array}{c}\mathrm{Br}-5 \\
(\mathrm{G})\end{array}$ & $\begin{array}{c}\mathrm{Br}-6 \\
(\mathrm{G})\end{array}$ & $\begin{array}{c}\mathrm{Br}-7 \\
(\mathrm{G})\end{array}$ & $\begin{array}{c}\mathrm{Br}-8 \\
(\mathrm{G})\end{array}$ & $\begin{array}{c}\text { Frequency } \\
(\mathrm{GHz})\end{array}$ \\
\hline$[001]$ & 3225.06 & 3252.64 & 3292.32 & 3320.76 & 3225.06 & 3252.64 & 3292.32 & 3320.76 & 9.59412 \\
\hline 10 & 3206.25 & 3248.24 & 3282.95 & 3317.37 & 3224.65 & 3252.15 & 3291.76 & 3319.64 & 9.59429 \\
\hline 20 & 3160.03 & 3215.12 & 3250.26 & 3296.41 & 3223.42 & 3250.26 & 3289.36 & 3316.89 & 9.59374 \\
\hline 30 & 3097.78 & 3159.21 & 3202.36 & 3259.20 & 3221.53 & 3247.56 & 3285.91 & 3312.13 & 9.5931 \\
\hline 40 & 3023.81 & 3090.27 & 3146.59 & 3209.39 & 3219.25 & 3243.89 & 3281.31 & 3305.96 & 9.59235 \\
\hline 50 & 2957.91 & 3027.78 & 3092.92 & 3161.14 & 3216.89 & 3240.23 & 3276.65 & 3299.98 & 9.5914 \\
\hline 60 & 2895.71 & 2969.70 & 3041.50 & 3114.24 & 3214.76 & 3236.49 & 3271.92 & 3293.64 & 9.5906 \\
\hline 70 & 2845.71 & 2922.41 & 2998.32 & 3074.78 & 3212.92 & 3233.70 & 3267.7 & 3287.86 & 9.5899 \\
\hline 80 & 2816.72 & 2894.83 & 2972.88 & 3051.32 & 3211.95 & 3231.14 & 3264.99 & 3284.37 & 9.58893 \\
\hline 110$]$ & 2806.08 & 2884.61 & 2963.70 & 3042.63 & 3211.82 & 3230.73 & 3264.17 & 3283.1 & 9.58918 \\
\hline
\end{tabular}


Table C.7. EPR angular dependence of $\mathrm{Cu}^{2+}$ taken from [110] to [-110]

\begin{tabular}{|c|c|c|c|c|c|c|c|c|c|}
\hline $\begin{array}{c}\text { Angle } \\
\left({ }^{\circ}\right)\end{array}$ & $\begin{array}{c}\mathrm{Br}-1 \\
(\mathrm{G})\end{array}$ & $\begin{array}{c}\mathrm{Br}-2 \\
(\mathrm{G})\end{array}$ & $\begin{array}{c}\mathrm{Br}-3 \\
(\mathrm{G})\end{array}$ & $\begin{array}{c}\mathrm{Br}-4 \\
(\mathrm{G})\end{array}$ & $\begin{array}{c}\mathrm{Br}-5 \\
(\mathrm{G})\end{array}$ & $\begin{array}{c}\mathrm{Br}-6 \\
(\mathrm{G})\end{array}$ & $\begin{array}{c}\mathrm{Br}-7 \\
(\mathrm{G})\end{array}$ & $\begin{array}{c}\mathrm{Br}-8 \\
(\mathrm{G})\end{array}$ & $\begin{array}{c}\text { Frequency } \\
(\mathrm{GHz})\end{array}$ \\
\hline$[110]$ & 2812.02 & 2890.4 & 2969.49 & 3048.71 & 3218.78 & 3237.69 & 3271.13 & 3290.04 & 9.609250 \\
\hline 5 & 2814.38 & 2893.07 & 2971.73 & 3050.88 & 3209.42 & 3240.52 & 3264.41 & 3292.01 & 9.609500 \\
\hline 10 & 2821.02 & 2899.12 & 2977.48 & 3055.83 & 3194.06 & 3221.79 & 3252.01 & 3289.72 & 9.608470 \\
\hline 20 & 2848.95 & 2925.27 & 3001.13 & 3077.14 & 3147.44 & 3183.97 & 3223.87 & 3270.94 & 9.608300 \\
\hline 30 & 2893.42 & 2966.5 & 3038.36 & 3110.37 & 3086.33 & 3129.91 & 3185.83 & 3238.23 & 9.609300 \\
\hline 40 & 2951.26 & 3017.87 & 3080.94 & 3151.97 & 3020.28 & 3080.94 & 3136.83 & 3196.27 & 9.609340 \\
\hline$[100]$ & 2983.97 & 3049.83 & 3110.99 & 3174.12 & 2983.97 & 3049.83 & 3110.99 & 3174.12 & 9.609350 \\
\hline 50 & 2951.41 & 3017.88 & 3080.95 & 3151.98 & 3020.09 & 3080.95 & 3137.00 & 3196.45 & 9.609350 \\
\hline 60 & 2892.41 & 2965.65 & 3037.53 & 3109.83 & 3087.84 & 3131.11 & 3186.75 & 3239.01 & 9.609350 \\
\hline 70 & 2848.55 & 2925.01 & 3000.87 & 3077.00 & 3148.31 & 3184.55 & 3224.33 & 3271.72 & 9.608960 \\
\hline 80 & 2820.66 & 2899.05 & 2977.4 & 3055.79 & 3194.86 & 3222.73 & 3252.52 & 3289.93 & 9.608900 \\
\hline 85 & 2813.73 & 2892.42 & 2971.38 & 3050.31 & 3210.09 & 3240.89 & 3264.08 & 3291.93 & 9.608900 \\
\hline
\end{tabular}


Table C.8. EPR angular dependence of interstitial $\mathrm{Li}^{+}$ion adjacent to a $\mathrm{Ti}^{3+}$ ion taken from [001] to [110]

\begin{tabular}{|c|c|c|c|c|c|c|c|c|c|}
\hline Angle $\left(^{\circ}\right)$ & $\mathrm{Br}-1(\mathrm{G})$ & $\mathrm{Br}-2(\mathrm{G})$ & $\mathrm{Br}-3(\mathrm{G})$ & $\mathrm{Br}-4(\mathrm{G})$ & $\mathrm{Br}-5(\mathrm{G})$ & $\mathrm{Br}-6(\mathrm{G})$ & $\mathrm{Br}-7(\mathrm{G})$ & $\mathrm{Br}-8(\mathrm{G})$ & Frequency $(\mathrm{GHz})$ \\
\hline$[001]$ & 3524.99 & 3525.78 & 3526.59 & 3527.38 & 3524.99 & 3525.78 & 3526.59 & 3527.38 & 9.53528 \\
\hline 10 & 3522.44 & 3523.15 & 3523.94 & 3524.71 & 3525.74 & 3526.49 & 3527.23 & 3528.00 & 9.535048 \\
\hline 20 & 3516.89 & 3517.59 & 3518.3 & 3518.82 & 3527.82 & 3528.4 & 3529.13 & 3529.88 & 9.537055 \\
\hline 30 & 3507.99 & 3508.47 & 3508.95 & 3509.38 & 3530.8 & 3531.32 & 3531.85 & 3532.36 & 9.535283 \\
\hline$[110]$ & 3464.42 & 3464.85 & 3465.19 & 3465.53 & & & & & 9.53996 \\
\hline
\end{tabular}

Table C.9. EPR angular dependence of interstitial $\mathrm{Li}^{+}$ion adjacent to a $\mathrm{Ti}^{3+}$ ion taken from [001] to [100]

\begin{tabular}{|c|c|c|c|c|c|c|c|c|c|}
\hline Angle $\left(^{\circ}\right)$ & $\mathrm{Br}-1(\mathrm{G})$ & $\mathrm{Br}-2(\mathrm{G})$ & $\mathrm{Br}-3(\mathrm{G})$ & $\mathrm{Br}-4(\mathrm{G})$ & $\mathrm{Br}-5(\mathrm{G})$ & $\mathrm{Br}-6(\mathrm{G})$ & $\mathrm{Br}-7(\mathrm{G})$ & $\mathrm{Br}-8(\mathrm{G})$ & Frequency $(\mathrm{GHz})$ \\
\hline$[001]$ & 3524.79 & 3525.6 & 3526.42 & 3527.24 & 3524.79 & 3525.6 & 3526.42 & 3527.24 & 9.535935 \\
\hline 15 & 3523.1 & 3523.69 & 3524.37 & 3524.99 & 3524.99 & 3525.82 & 3526.61 & 3527.36 & 9.53682 \\
\hline 20 & 3521.35 & 3521.91 & 3522.46 & 3523.03 & 3524.82 & 3525.62 & 3526.41 & 3527.17 & 9.53719 \\
\hline 30 & & & & & 3524.16 & 3524.96 & 3525.73 & 3526.6 & 9.537738 \\
\hline 40 & 3507.88 & & & 3508.4 & 3522.58 & 3523.37 & 3524.14 & 3524.94 & 9.538382 \\
\hline 50 & 3501.39 & 3501.92 & 3502.44 & 3502.98 & 3521.7 & 3522.55 & 3523.38 & 3524.18 & 9.539021 \\
\hline 60 & 3495.21 & 3496.04 & 3496.93 & 3497.79 & 3521.06 & 3521.85 & 3522.63 & 3523.43 & 9.54011 \\
\hline 70 & 3490.74 & 3491.83 & 3492.86 & 3493.93 & 3520.26 & 3521.1 & 3521.9 & 3522.69 & 9.54012 \\
\hline 80 & 3488.01 & 3489.27 & 3490.43 & 3491.63 & 3519.88 & 3520.7 & 3521.48 & 3522.31 & 9.540381 \\
\hline$[100]$ & 3487.91 & 3489.15 & 3490.4 & 3491.66 & 3520.02 & 3520.85 & 3521.6 & 3522.41 & 9.540636 \\
\hline
\end{tabular}


Table C.10. EPR angular dependence of interstitial $\mathrm{Li}^{+}$ion adjacent to a $\mathrm{Ti}^{3+}$ ion taken from [100] to [110]

\begin{tabular}{|c|c|c|c|c|c|c|c|c|}
\hline Angle $\left(^{\circ}\right)$ & $\mathrm{Br}-1(\mathrm{G})$ & $\mathrm{Br}-2(\mathrm{G})$ & $\mathrm{Br}-3(\mathrm{G})$ & $\mathrm{Br}-4(\mathrm{G})$ & $\mathrm{Br}-5(\mathrm{G})$ & $\mathrm{Br}-6(\mathrm{G})$ & $\mathrm{Br}-7(\mathrm{G})$ & $\mathrm{Br}-8(\mathrm{G})$ \\
\hline$[100]$ & 3485.17 & 3487.1 & 3488.4 & 3489.86 & 3485.17 & 3487.1 & 3488.4 & 3489.86 \\
\hline 5 & 3477.51 & 3478.75 & 3479.05 & 3481.27 & 3491.4 & 3492.63 & 3493.82 & 3495.02 \\
\hline 10 & 3470.18 & 3471.75 & 3472.93 & 3474.12 & 3500.29 & 3500.8 & 3501.46 & 3502.12 \\
\hline 20 & 3461.99 & 3463.05 & 3464.07 & 3465.11 & 3513.98 & 3515.31 & 3516.18 & 3517.08 \\
\hline 30 & 3456.6 & 3457.44 & 3458.25 & 3459.12 & 3527.33 & 3527.96 & 3528.52 & 3529.05 \\
\hline 40 & 3456.83 & 3457.77 & 3457.92 & 3458.45 & & & & \\
\hline$[110]$ & 3458.4 & 3458.78 & 3459.11 & 3459.48 & & & & \\
\hline
\end{tabular}

Table C.10. Continued

\begin{tabular}{|c|c|c|c|c|c|c|c|c|c|}
\hline $\begin{array}{c}\text { Angle } \\
\left({ }^{\circ}\right)\end{array}$ & $\mathrm{Br}-9(\mathrm{G})$ & $\begin{array}{c}\mathrm{Br}-10 \\
(\mathrm{G})\end{array}$ & $\begin{array}{c}\mathrm{Br}-11 \\
(\mathrm{G})\end{array}$ & $\begin{array}{c}\mathrm{Br}-12 \\
(\mathrm{G})\end{array}$ & $\begin{array}{c}\mathrm{Br}-13 \\
(\mathrm{G})\end{array}$ & $\begin{array}{c}\mathrm{Br}-14 \\
(\mathrm{G})\end{array}$ & $\begin{array}{c}\mathrm{Br}-15 \\
\text { (G) }\end{array}$ & $\begin{array}{c}\mathrm{Br}-16 \\
\text { (G) }\end{array}$ & Frequency $(\mathrm{GHz})$ \\
\hline$[100]$ & 3516.76 & 3517.56 & 3518.35 & 3519.15 & 3516.76 & 3517.56 & 3518.35 & 3519.15 & 9.535556 \\
\hline 5 & 3509.1 & 3509.92 & 3510.67 & 3511.34 & 3523.13 & 3523.93 & 3524.76 & 3525.57 & 9.531688 \\
\hline 10 & 3499.23 & 3500.29 & 3501.46 & 3502.59 & 3528.91 & 3529.72 & 3530.52 & 3531.33 & 9.531136 \\
\hline 20 & 3486.25 & 3486.74 & 3487.17 & 3487.67 & 3538.97 & 3539.72 & 3540.38 & 3541.13 & 9.528744 \\
\hline 30 & & & & & & & & & 9.529144 \\
\hline 40 & & & & & & & & & 9.524605 \\
\hline [110] & & & & & & & & & 9.522114 \\
\hline
\end{tabular}

